Supporting Information

\title{
Dual Role of Pyrrolidine and Cooperative Pyrrolidine/Pyrrolidinium Effect in Nitrone Formation
}

Sara Morales, Fernando. G. Guijarro, Inés Alonso, José Luis García Ruano* and M. Belén Cid* Department of Organic Chemistry. Universidad Autónoma de Madrid. Cantoblanco 28049, Madrid, Spain

e-mail: belen.cid@uam.es, joseluis.garcia.ruano@uam.es

\section{Table of Contents}

1. ADDITIONAL OPTIMIZATION STUDIES

S1

2. COMPARISON OF CONDITIONS PREVIOUSLY REPORTED FOR THE PREPARED COMPOUNDS S3

3. ${ }^{1} \mathrm{H}$-NMR EXPERIMENTS

S4

- Liberation of the hydrochloride $\mathbf{2} \mathbf{b} \cdot \mathbf{H C l}$ using pyrrolidine

- Study of the optimal pyrrolidine amount and cooperative pyrrolidine/pyrrolidinium effect.

- Representative ${ }^{1} \mathrm{H}$ NMR spectra of monitorization of the synthesis of 3ab under different conditions

- Monitorization of the evolution of iminium ion $\mathbf{4}$ in the presence of $\mathbf{2 b} \cdot \mathbf{H C l}$

4. EXPERIMENTAL SECTION

- General remarks

- Synthesis of nitrones: general procedures

5. REFERENCES

6. THEORETICAL CALCULATIONS

7. ${ }^{1}$ H NMR AND ${ }^{13}$ C SPECTRA 


\section{ADDITIONAL OPTIMIZATION STUDIES}

+ Effect of substoichiometric amount of pyrrolidine, other bases and solvents

Apart from the results gathering in Table 1, other reaction conditions were investigated (Table S1).

The use of less amount of pyrrolidine (entry 1 ) does not allow complete conversion in reasonable reaction times. Nevertheless, it points out the catalytic character of the process.

The combination of other bases to liberate the hydrochloride along with catalytic pyrrolidine (entries 2-4) gave lower conversions than those indicated in Table 1.

3ab could also be prepared in different solvents, like $\mathrm{CH}_{3} \mathrm{CN}$, EtOAc or 2-Me-THF, in almost quantitative yields using 1 equiv of pyrrolidine. Nevertheless, reaction times were similar (entries 5-7) to those using $\mathrm{H}_{2} \mathrm{O} / \mathrm{MeOH}$ (conditions A).

Table S1.

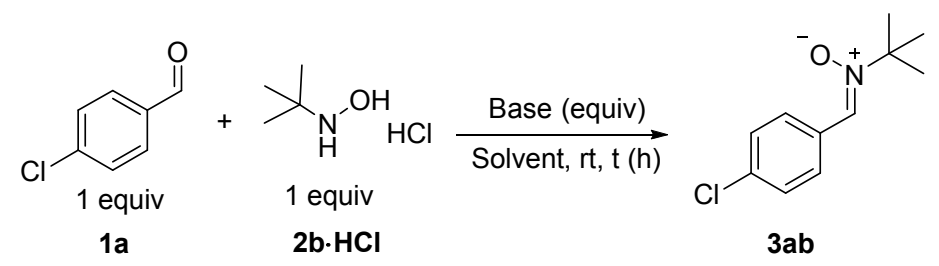

\begin{tabular}{ccccc}
\hline Entry & Base (equiv) & Solvent & $\mathrm{t}(\mathrm{h})$ & Conv.(\%) \\
\hline 1 & Pyrrolidine (0.5) & $\mathrm{H}_{2} \mathrm{O} / \mathrm{MeOH}$ & $16(72)$ & $63(63)$ \\
2 & Pyrrolidine (0.1) $+\mathrm{Na}_{2} \mathrm{CO}_{3}(1)$ & $\mathrm{H}_{2} \mathrm{O} / \mathrm{MeOH}$ & 6 & 83 \\
3 & Pyrrolidine (0.1) $+\mathrm{NaHCO}_{3}(1)$ & $\mathrm{H}_{2} \mathrm{O} / \mathrm{MeOH}$ & 6 & 70 \\
4 & Pyrrolidine (0.1) $+\mathrm{Et}_{3} \mathrm{~N}(1)$ & $\mathrm{H}_{2} \mathrm{O} / \mathrm{MeOH}$ & 6 & 67 \\
5 & Pyrrolidine (1) & $\mathrm{CH}_{3} \mathrm{CN}$ & 16 & 100 \\
6 & Pyrrolidine (1) & $\mathrm{EtOAc}$ & 16 & 100 \\
7 & Pyrrolidine (1) & 2-Me-THF & 30 & 100 \\
\hline
\end{tabular}




\section{COMPARISON OF CONDITIONS PREVIOUSLY REPORTED FOR THE PREPARED COMPOUNDS}

Tables S2 and S3 show the conditions that reported the best yield for each compound using the condensation approach and the results obtained by us. The yields obtained with our method under both conditions $A$ and $B$ are comparable or superior to the best described to obtain a given nitrone, pointing out the generality, simplicity and economy of pyrrolidine mediated strategy with respect to the methods so far reported

Table S2. Comparison of methods to prepare $N$-tert-butylnitrones.

\begin{tabular}{|c|c|c|c|c|c|c|}
\hline & $\begin{array}{l}\text { Cond } \mathrm{A} \\
\text { Pyrrolidine (1 equiv), } \\
\mathrm{H}_{2} \mathrm{O} / \mathrm{MeOH} \\
\text { Cond } \mathrm{B} \text { : } \\
\text { yrrolidine ( } 1.2 \text { equiv), } \\
\text { DCM }\end{array}$ & ${ }_{3}$ & & 1 & 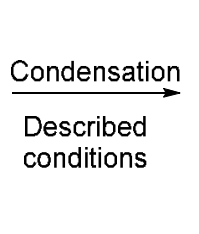 & 3 \\
\hline Ent. & ${\text { Aldehyde } \mathrm{R}^{1}}^{1}$ & $\begin{array}{l}\text { Yield A } \\
(\mathrm{t}(\mathrm{h}))\end{array}$ & $\begin{array}{l}\text { Yield B } \\
(\mathrm{t}(\min ))\end{array}$ & \multicolumn{3}{|c|}{ Condensation methods } \\
\hline 1 & $2-\mathrm{OH}-5-\mathrm{ClC}_{6} \mathrm{H}_{3}$ & $98(6)$ & - & \multirow{3}{*}{\multicolumn{2}{|c|}{$\begin{array}{l}\text { i) } t \text { - } \mathrm{BuNO}_{2}, \mathrm{Zn}, \mathrm{NH}_{4} \mathrm{Cl}, \mathrm{H}_{2} \mathrm{O}, 15 \mathrm{~h} ; \\
\text { ii) } 1 \mathrm{I}+\mathrm{HCl}_{2} \mathrm{H} \mathrm{O} / \mathrm{MeOH}, \text { reflujo; } \mathrm{CC} \\
\text { i) } t-\mathrm{BuNO}_{2}, \mathrm{Zn}, \mathrm{NH}_{4} \mathrm{Cl}, \mathrm{H}_{2} \mathrm{O}, 15 \mathrm{~h} ; \\
\text { ii) } 1 \mathrm{~m}, p-\mathrm{TSA}, \text { Benzene, reflux; } \mathrm{CC} \\
\text { 1h }+t \text { - } \mathrm{BuNO}_{2} \text { ( } 2 \text { equiv), } \mathrm{Zn} \text { ( } 4 \text { equiv), } \mathrm{AcOH} \\
\text { (6 equiv), } 22 \mathrm{~h} ; \mathrm{CC}\end{array}$}} & $99^{1}$ \\
\hline 2 & $2-\mathrm{OH}-5-\mathrm{NO}_{2} \mathrm{C}_{6} \mathrm{H}_{3}$ & $96(6.5)$ & - & & & $75^{1}$ \\
\hline 3 & $o-\mathrm{BrC}_{6} \mathrm{H}_{4}$ & $92(16)$ & - & & & \\
\hline 4 & $i$-Bu & $99(5.5)$ & $92(3)$ & \multicolumn{2}{|c|}{$1 \mathrm{~s}+t-\mathrm{BuNO}_{2}, \mathrm{Zn}, \mathrm{AcOH}, \mathrm{EtOH}: \mathrm{H}_{2} \mathrm{O}$} & $95^{3}$ \\
\hline 5 & $p-\mathrm{NO}_{2} \mathrm{C}_{6} \mathrm{H}_{4}$ & $95(21)$ & $\begin{array}{l}100 \\
(34)\end{array}$ & \multirow{4}{*}{\multicolumn{2}{|c|}{ 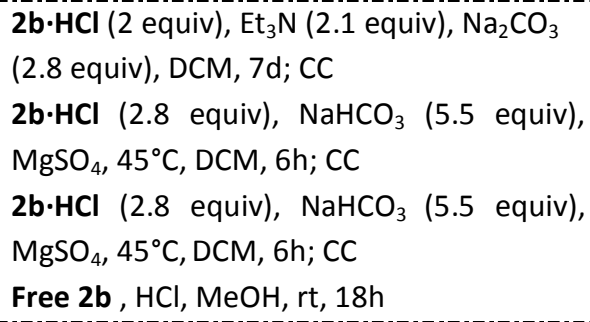 }} & $96^{4}$ \\
\hline 6 & $\mathrm{C}_{6} \mathrm{H}_{4} \mathrm{CH}=\mathrm{CH}-$ & $100(4)$ & $98(60)$ & & & $75^{5}$ \\
\hline 7 & Cy & $99(5.5)$ & $98(3)$ & & & $73^{5}$ \\
\hline 8 & $\mathrm{Ph}(\mathrm{Me}) \mathrm{CH}-$ & $96(5.5)$ & $80(12)$ & & & $71^{6}$ \\
\hline 9 & $\mathrm{C}_{6} \mathrm{H}_{5}$ & $91(6)$ & $97(15)$ & \multirow{2}{*}{\multicolumn{2}{|c|}{$\begin{array}{l}\text { Ball mill, } \mathrm{NaHCO}_{3} \text { ( } 1 \text { equiv), } 2 \mathrm{~h} \\
\mathrm{MW}, 1 \mathrm{c}(1.2 \text { equiv), AcONa (1.2 equiv), } \\
137^{\circ} \mathrm{C}, 3 \mathrm{~min} ; \mathrm{CC}\end{array}$}} & $100^{7}$ \\
\hline 10 & $p-\mathrm{MeOC}_{6} \mathrm{H}_{4}$ & $100(21)$ & $94(45)$ & & & $91^{8}$ \\
\hline 11 & $p-\mathrm{ClC}_{6} \mathrm{H}_{4}$ & $94(16)$ & $98(20)$ & \multicolumn{2}{|c|}{$\begin{array}{l}\mathrm{MW}, 1 \mathrm{a} \text { (1.2 equiv), } \mathrm{AcONa} \text { (1.2 equiv), } \\
143^{\circ} \mathrm{C}, 2 \mathrm{~min} ; \mathrm{CC}\end{array}$} & $85^{8}$ \\
\hline 12 & 2-OH-4-MeOC ${ }_{6} \mathrm{H}_{3}$ & $100(16)$ & $98(3)$ & \multicolumn{2}{|c|}{$\mathrm{MW}$, Ionic liq. (1 equiv), $70^{\circ} \mathrm{C}, 3.5 \mathrm{~min}$; $\mathrm{RC}$} & $99^{9}$ \\
\hline 13 & 2-pyridyl & $96(7)$ & $100(8)$ & \multirow{3}{*}{\multicolumn{2}{|c|}{$\begin{array}{l}\mathrm{MW} \text {, lonic liq. ( } 1 \text { equiv), } 70^{\circ} \mathrm{C}, 5 \text { min; } \mathrm{RC} \\
\mathrm{MW}, \mathbf{2 b} \cdot \mathrm{HCl}\left(2 \text { equiv), } \mathrm{Et}_{3} \mathrm{~N}(2 \text { equiv), }\right. \\
\mathrm{Na}_{2} \mathrm{SO}_{4}\left(3 \text { equiv), } \mathrm{THF}, 80^{\circ} \mathrm{C}, 2 \mathrm{~h} ; \mathrm{CC}\right. \\
\mathrm{MW}, 2 \mathrm{~b} \cdot \mathrm{HCl} \text { ( } 2 \text { equiv), } \mathrm{Et}_{3} \mathrm{~N} \mathrm{(2} \mathrm{equiv),} \\
\mathrm{Na}_{2} \mathrm{SO}_{4} \text { (3 equiv), THF, } 80^{\circ} \mathrm{C}, 33 \mathrm{~h} ; \mathrm{CC}\end{array}$}} & $93^{9}$ \\
\hline 14 & 2-Cl-3-pyridyl & $92(16)$ & - & & & $99^{10}$ \\
\hline 15 & 3-indolyl & $100(29)$ & $92(240)$ & & & $76^{10}$ \\
\hline 16 & $o-\mathrm{ClC}_{6} \mathrm{H}_{4}$ & $100(16)$ & $100(20)$ & \multicolumn{3}{|c|}{ Not described } \\
\hline 17 & $o-\mathrm{MeOC}_{6} \mathrm{H}_{4}$ & $100(16)$ & $100(6)$ & \multicolumn{3}{|c|}{ Not described } \\
\hline 18 & 2-OH-5- $\mathrm{MeC}_{6} \mathrm{H}_{3}$ & $90(7)$ & - & \multicolumn{3}{|c|}{ Not described } \\
\hline 19 & 2-OH-3-MeOC ${ }_{6} \mathrm{H}_{3}$ & $100(8)$ & $100(9)$ & \multicolumn{3}{|c|}{ Not described } \\
\hline 20 & $n-\mathrm{Bu}$ & 100(5.5) & $100(3)$ & \multicolumn{3}{|c|}{ Not described } \\
\hline
\end{tabular}


Table S3. Comparison of methods to prepare $\mathrm{N}$-phenyl and other $\mathrm{N}$-alkyl-nitrones

\begin{tabular}{|c|c|c|c|c|c|c|c|}
\hline $\begin{array}{l}\text { Pyrr } \\
\text { Pyrro }\end{array}$ & $\begin{array}{l}\text { Cond } A \\
\text { lidine (1 equiv), } \\
\mathrm{H}_{2} \mathrm{O} / \mathrm{MeOH} \\
\text { Cond } \mathrm{B} \text { : } \\
\text { idine ( } 1.2 \text { equiv) } \\
\text { DCM }\end{array}$ & , & & 1 & 2a, 2c-e. $\mathrm{HCl}$ & 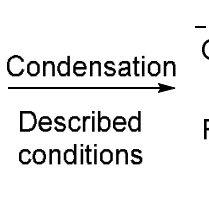 & $\mathrm{R}^{1}{ }_{3}^{-1}$ \\
\hline \multirow[t]{2}{*}{ Entry } & Aldehyde & $2 R^{2}$ & Yield A & Yield B & \multicolumn{3}{|c|}{ Condensation methods } \\
\hline & $\mathrm{R}^{1}$ & & $(t(h))$ & $(t(\min ))$ & Conditions $^{\mathrm{a}}$ & & Yield (\%) \\
\hline 1 & $p-\mathrm{ClC}_{6} \mathrm{H}_{4}$ & $2 \mathrm{cBn}$ & 99 (9) & $99(3)$ & $\begin{array}{l}\text { Free } 2 \mathrm{c} \text { ( } 1.1 \text { equiv) } \\
\text { equiv), DCM, } \mathrm{rt}, 16\end{array}$ & $\begin{array}{l}\mathrm{MgSO}_{4}(1.3 \\
; \mathrm{CC}\end{array}$ & $55^{11}$ \\
\hline 2 & $t-\mathrm{Bu}$ & $2 \mathrm{cBn}$ & $84(6.5)$ & - & Free $2 \mathrm{c}, \mathrm{MgSO}_{4}$, Et & $\mathrm{O}, \mathrm{rt}, 15 \mathrm{~h} ; \mathrm{RC}$ & $85^{12}$ \\
\hline 3 & $p-\mathrm{ClC}_{6} \mathrm{H}_{4}$ & 2d Me & $93(6)$ & $93(3)$ & $\begin{array}{l}\mathrm{Et}_{3} \mathrm{~N} \text { (1 equiv), } \mathrm{NaS} \\
\mathrm{CC}\end{array}$ & $\mathrm{D}_{4}, \mathrm{DCM}, \mathrm{rt}, 4 \mathrm{~h}$ & $93^{13}$ \\
\hline 4 & $\mathrm{PhCH}=\mathrm{CH}$ & 2d Me & $84(3)$ & - & $\begin{array}{l}2 \mathrm{~d} \cdot \mathrm{HCl}(2.8 \text { equiv), } \\
\text { equiv), } \mathrm{MgSO}_{4}, \mathrm{DC}\end{array}$ & $\begin{array}{l}\mathrm{VaHCO}_{3}(5.5 \\
1,45^{\circ} \mathrm{C}, 6 \mathrm{~h} ; \mathrm{CC}\end{array}$ & $82^{5}$ \\
\hline 5 & $p-\mathrm{ClC}_{6} \mathrm{H}_{4}$ & $2 \mathrm{e} C y$ & $97(16)$ & $100(3)$ & Not de & cribed & \\
\hline 6 & $\mathrm{Ph}(\mathrm{Me}) \mathrm{CH}$ & $2 e \mathrm{Cy}$ & - & $94(3)$ & $\begin{array}{l}1 \mathrm{u} \text { (1.1 equiv), AcO } \\
\mathrm{H}_{2} \mathrm{O} / \mathrm{EtOH}, \mathrm{rt}, 24 \mathrm{~h} ;\end{array}$ & $\begin{array}{l}\mathrm{Na}(1.6 \text { equiv), } \\
\mathrm{XC}\end{array}$ & $73^{14}$ \\
\hline 7 & $p-\mathrm{ClC}_{6} \mathrm{H}_{4}$ & 2a Ph & $92(48)$ & $96(15)$ & EtOH, rt, 16h; CC & & $94^{15}$ \\
\hline
\end{tabular}




\section{3. ${ }^{1} \mathrm{H}$ NMR EXPERIMENTS}

+ Liberation of the hydrochloride $\mathbf{2 b} \cdot \mathrm{HCl}$ using pyrrolidine

We observed the instantaneous liberation of hydroxylamine $\mathbf{2} \mathbf{b} \cdot \mathbf{H C l}$ when it was treated with pyrrolidine (1 equiv).

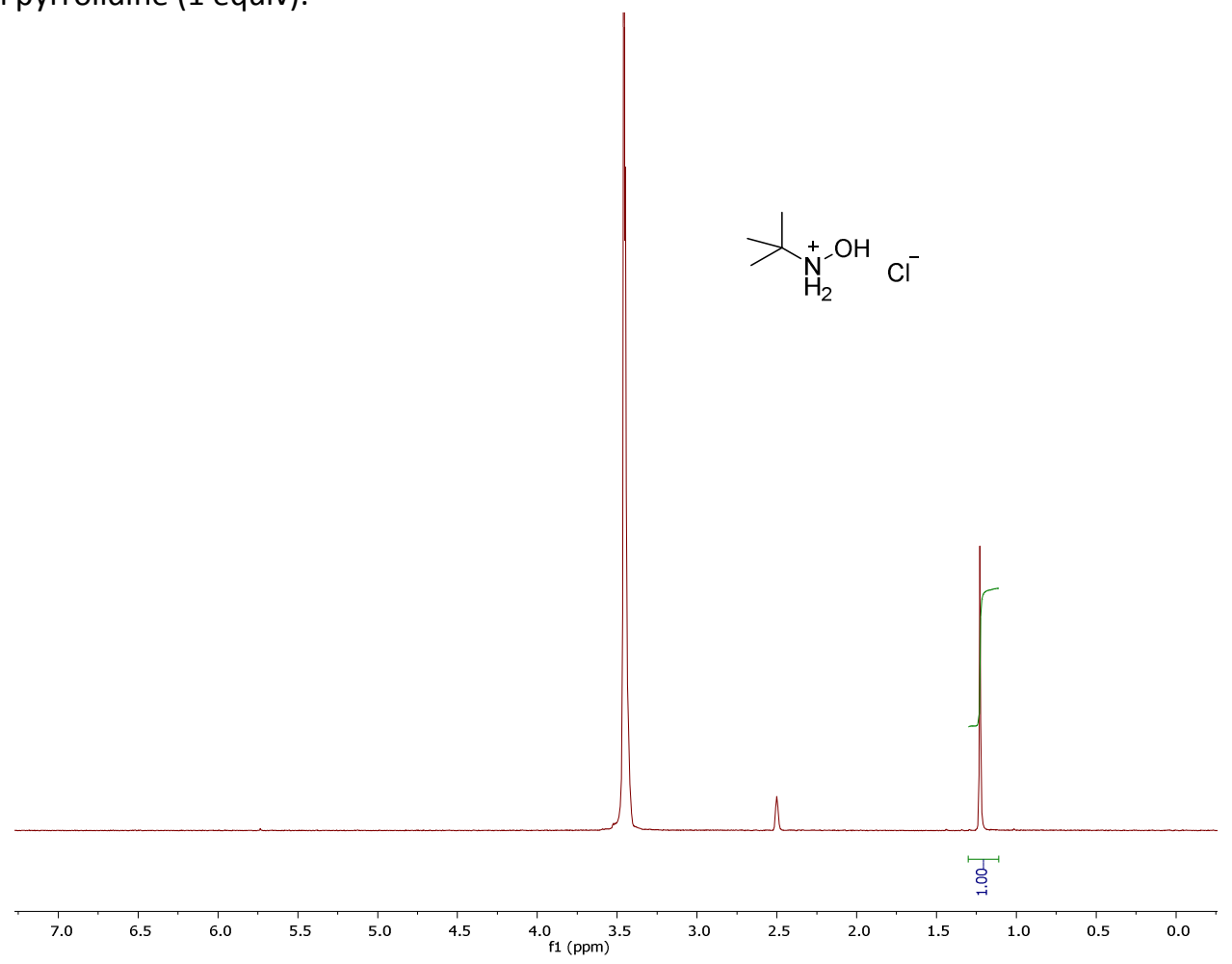

${ }^{1} \mathrm{H}$ NMR recorded in DMSO- $d_{6}$ at $\mathrm{t}=4 \mathrm{~min}$

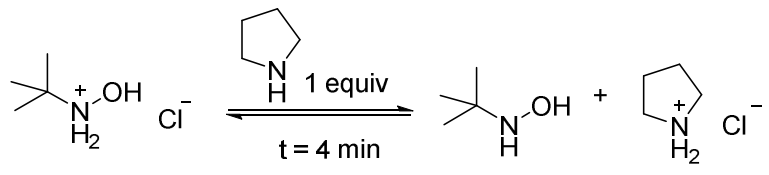

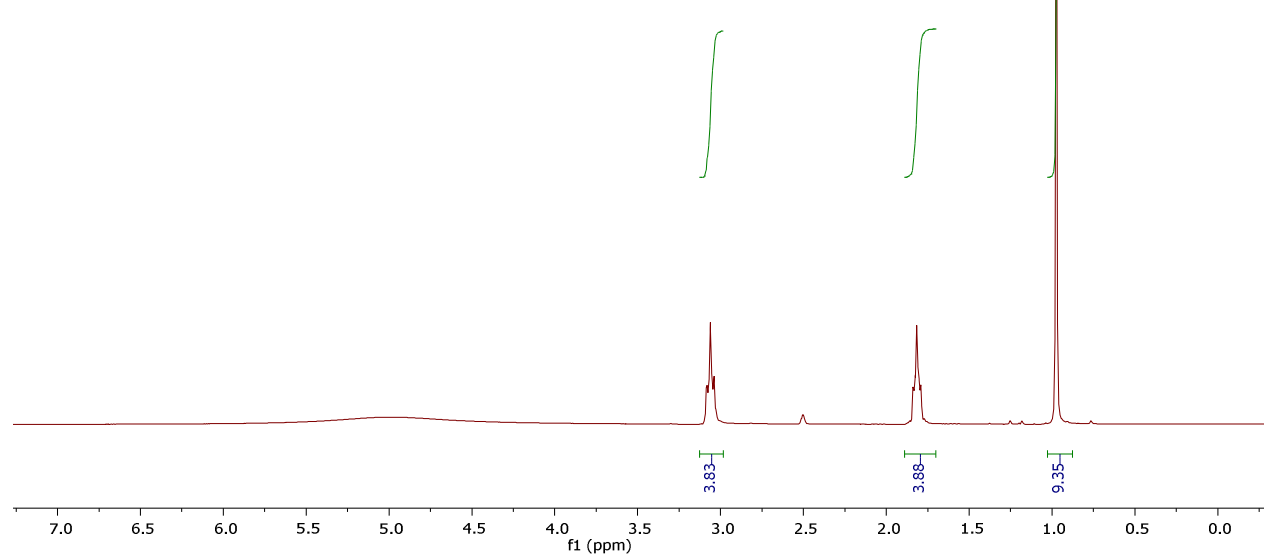


+ Study of the optimal pyrrolidine amount and cooperative pyrrolidine/pyrrolidinium effect.

${ }^{1} \mathrm{H}$ NMR study of the evolution of species in the formation of nitrones $\mathbf{3} a \boldsymbol{b}$ and $\mathbf{3 a a}$.

Table S4 summarized our efforts addressed to confirm the iminium catalysis and to understand several aspects, such as the optimal amount of pyrrolidine and the role of the pyrrolidinium ion generated when hydroxylamine hydrochlorides are used $(\mathbf{2} \cdot \mathbf{H C l})$. For these purposes, we tried to identify the intermediate species by monitoring the evolution of the reaction between $\mathbf{1} \mathbf{a}$ and $\mathbf{2} \mathbf{b}$ or $\mathbf{2} \mathbf{b} \cdot \mathbf{H C l}$ in $\mathrm{MeOH}-d_{4}$ and $\mathrm{CD}_{2} \mathrm{Cl}_{2}$ (respectively used in conditions $\mathrm{A}$ and $\mathrm{B}$ ) by ${ }^{1} \mathrm{H}-\mathrm{NMR}$, in the presence of different amounts of pyrrolidine. Although we paid special attention to the detection of aminal III, corresponding to the attack of $\mathbf{2 b}$ to the iminium ion II (Scheme 1), as shown in Table S4, only the signals corresponding to the hemiacetal IV, hemiaminal $\mathbf{V}$ (both in $\mathrm{MeOH}-d_{4}$ ), and aminal VI (in $\mathrm{CD}_{2} \mathrm{Cl}_{2}$ ) were detected under some conditions. The presence of $\mathbf{V}$ and $\mathbf{V} \mathbf{l}$, again support the formation of the iminium ion as intermediate.

Table S4. Ratio of species in the formation of nitrones $3 a b$ and $3 a a$.

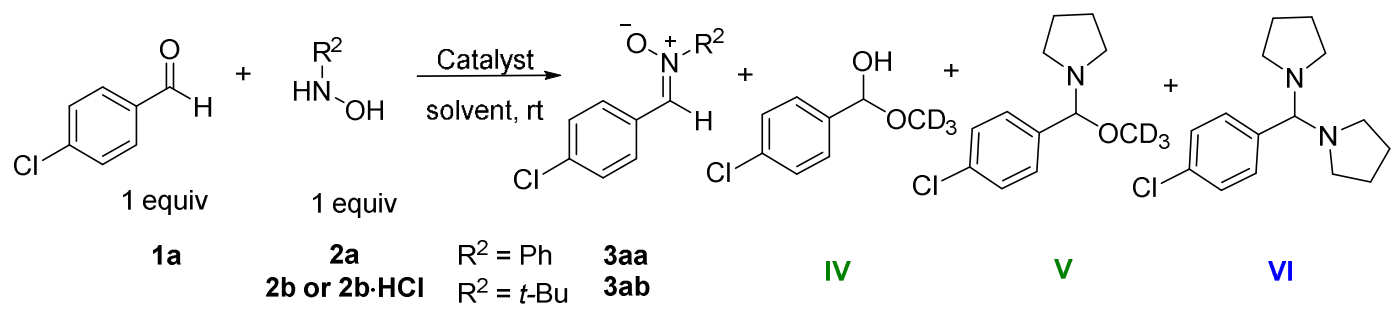

\begin{tabular}{|c|c|c|c|c|c|c|c|c|c|}
\hline \multirow[t]{2}{*}{ Entry } & \multirow[t]{2}{*}{$\mathrm{Nu}$} & \multirow[t]{2}{*}{ Solvent } & \multirow{2}{*}{$\begin{array}{l}\text { Catalyst } \\
\text { (equiv) }\end{array}$} & \multirow{2}{*}{$\begin{array}{l}\text { Time } \\
(\min )\end{array}$} & \multicolumn{5}{|c|}{ Ratio (\%) } \\
\hline & & & & & 1a & 3 & IV & v & VI \\
\hline 1 & $2 \mathbf{b}$ & $\mathrm{MeOH}-d_{4}$ & -- & 24 & 80 & 0 & 20 & & \\
\hline 2 & $2 b$ & $\mathrm{MeOH}-d_{4}$ & -- & 64 & 80 & 0 & 20 & & \\
\hline 3 & $2 b$ & $\mathrm{MeOH}-d_{4}$ & Pyrrolidine (0.2) & 24 & 52 & 21 & 15 & 12 & \\
\hline 4 & $2 b$ & $\mathrm{MeOH}-d_{4}$ & Pyrrolidine (0.2) & 64 & 27 & 54 & 7 & 12 & \\
\hline 5 & $2 \mathrm{~b} \cdot \mathrm{HCl}$ & $\mathrm{MeOH}-d_{4}$ & Pyrrolidine (1) & 11 & 26 & 68 & 6 & nd & -- \\
\hline 6 & $2 \mathrm{~b} \cdot \mathrm{HCl}$ & $\mathrm{MeOH}-d_{4}$ & Pyrrolidine (1.2) & 4 & 15 & 79 & 3 & 3 & -- \\
\hline 7 & $2 b \cdot \mathrm{HCl}$ & $\mathrm{MeOH}-d_{4}$ & Pyrrolidine (2) & 2 & 26 & 56 & nd & 18 & -- \\
\hline 8 & $2 \mathrm{~b} \cdot \mathrm{HCl}$ & $\mathrm{MeOH}-d_{4}$ & Pyrrolidine (2) & 30 & 7 & 87 & nd & 6 & \\
\hline 9 & $2 \mathrm{~b} \cdot \mathrm{HCl}$ & $\mathrm{CD}_{2} \mathrm{Cl}_{2}$ & Pyrrolidine (1) & 11 & 36 & 64 & - & - & nd \\
\hline 10 & $2 b \cdot \mathrm{HCl}$ & $\mathrm{CD}_{2} \mathrm{Cl}_{2}$ & Pyrrolidine (1.2) & 4 & 15 & 85 & - & - & nd \\
\hline 11 & $2 \mathrm{~b} \cdot \mathrm{HCl}$ & $\mathrm{CD}_{2} \mathrm{Cl}_{2}$ & Pyrrolidine (2) & 2 & 21 & 67 & - & - & 12 \\
\hline 12 & $2 \mathrm{~b} \cdot \mathrm{HCl}$ & $\mathrm{CD}_{2} \mathrm{Cl}_{2}$ & Pyrrolidine (2) & 24 & 5 & 93 & & & 2 \\
\hline 13 & $2 a$ & $\mathrm{CD}_{2} \mathrm{Cl}_{2}$ & --- & 15 & & 22 & & & \\
\hline 14 & $2 a$ & $\mathrm{CD}_{2} \mathrm{Cl}_{2}$ & Pyrrolidine (0.2) & 15 & -- & 75 & -- & -- & -- \\
\hline 15 & $2 a$ & $\mathrm{CD}_{2} \mathrm{Cl}_{2}$ & $\begin{array}{l}\text { Pyrrolidine } \cdot \mathrm{HCl}(1 \\
+ \text { Pyrrolidine }(0.2)\end{array}$ & 15 & -- & 100 & -- & -- & -- \\
\hline
\end{tabular}


The formation of nitrone $\mathbf{3} \mathbf{a b}$ with the previously liberated $N$ - $t$-butyl hydroxylamine $\mathbf{2} \mathbf{b}^{16}$ was not observed in $\mathrm{MeOH}-d_{4}$, detecting exclusively signals of the starting compounds (1a and $\mathbf{2} \mathbf{b}$ ) along with those of the hemiacetal IV after 24-64 min (entries 1-2) (see spectrum below). The catalytic effect via iminium activation is evidenced in entry 3 , as the use of 0.2 equiv of pyrrolidine allows the detection of intermediate $\mathbf{V}$ and the formation of the nitrone 3ab. The composition of this mixture evolved with time, and, after 64 minutes, the proportion of the nitrone 3aa increased (54\%) whereas those of the starting aldehyde 1a and the hemiacetal IV decreased, indicating the progress of the reaction (entry 4) (see spectrum below).

Starting from the corresponding hydrochloride $\mathbf{2} \mathbf{b} \cdot \mathbf{H C l}$, the use of 1 equiv of pyrrolidine (entry 5) should produce 1 equiv of pyrrolidinium in the reaction media. These conditions visibly increase the effectiveness towards nitrone evolution; $3 a b$ is formed with $68 \%$ conversion after $11 \mathrm{~min}$ and also a decrease of the ratio of the species IV and $\mathbf{V}$ was observed. The coexistence of 1 equiv of pyrrolidinium and 0.2 equiv of pyrrolidine (entry 6) was clearly the most efficient catalytic system, reaching $79 \%$ of conversion towards nitrone 3ab in just 4 min. The comparison of results in entries 3-6 put forward a cooperative pyrrolidine/pyrrolidinium effect.

An increase of the amount of pyrrolidine to 2 equiv (entry 7) drives to a fast disappearance of the aldehyde but not a complete conversion to the nitrone. The largest ratio of hemiaminal $\mathbf{V}$ (24\% after $2 \mathrm{~min}$ ), suggests a greater iminium ion concentration, in turn is due to the higher concentration of free pyrrolidine, but also involved longer reaction times to achieve complete formation of the nitrone $\mathbf{3} \mathbf{a b}$ (after $30 \mathrm{~min}, 6 \%$ of $\mathbf{V}$ is still observed, entry 8 ). In a similar way, using $\mathrm{CD}_{2} \mathrm{Cl}_{2}$ as solvent, aminal VI was only detected when 2 equiv. of pyrrolidine were added (entry 11), which evolved slowly to $3 a b$ (after $24 \mathrm{~min}, 2 \%$ of $\mathbf{V I}$ is still detected, entry 12). Therefore, 1.2 equiv (entry 10 ) is the optimal amount of pyrrolidine, because it minimizes the formation of the specie VI, which is out of the catalytic cycle, in equilibrium with the iminium ion, slowing down the complete transformation of the aldehyde 1a into the desired nitrone 3ab. We reasoned that the longer reaction times observed in $\mathrm{MeOH}$ (compare entries 6 and 10) could be partially due to the formation of hemiacetal IV, which is also out of the catalytic cycle.

The cooperative effect of pyrrolidinium/ pyrrolidine was also confirmed with experiments shown in entries 11-13 using free $N$-phenyl hydroxylamine 2a. Entry 15 shows the result obtained when trying to mimic the optimized conditions $B$ as if the reaction was performed with the corresponding hydrochloride using a mixture of 1 equiv of pyrrolidinium chloride (preformed) and 0.2 of pyrrolidine as catalysts. The presence of pyrrolidinium chloride provided a complete conversion into 3aa in only 15 minutes (compare time with entry 14). 
+ Table S5. ${ }^{1} \mathrm{H}$ NMR monitorization of 3ab formation using $\mathrm{Et}_{3} \mathrm{~N}$ and pyrrolidine in $\mathrm{MeOH}-\mathrm{d} 4$.

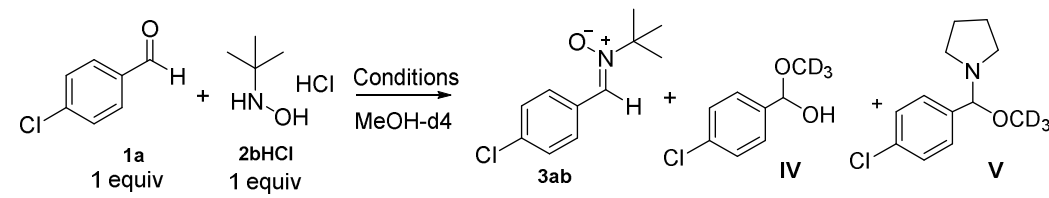

\begin{tabular}{|c|c|c|c|c|c|c|}
\hline Entry & $\begin{array}{l}\text { Reagent } \\
\text { (equiv) }\end{array}$ & Time & $\begin{array}{l}3 a b \\
(\%) \\
\end{array}$ & $\begin{array}{l}1 a \\
(\%) \\
\end{array}$ & $\begin{array}{l}\text { IV } \\
(\%) \\
\end{array}$ & $\begin{array}{l}V \\
\text { (\%) } \\
\end{array}$ \\
\hline 1 & Pyrrolidine (1) & $4 \min$ & 68 & 27 & 5 & nd \\
\hline 2 & Pyrrolidine (1) & $11 \mathrm{~min}$ & 69 & 26 & 6 & nd \\
\hline 3 & Pyrrolidine (1) & $21 \mathrm{~min}$ & 70 & 25 & 5.5 & nd \\
\hline 4 & Pyrrolidine (1) & $2 \mathrm{~h} 40 \mathrm{~min}$ & 83 & 14 & 3 & nd \\
\hline 5 & Pyrrolidine (1) & $6.5 \mathrm{~h}$ & 100 & 0 & 0 & \\
\hline 6 & Pyrrolidine (1.2) & $4 \mathrm{~min}$ & 79 & 15 & 3 & 3 \\
\hline 7 & Pyrrolidine (1.2) & $3.5 \mathrm{~h}$ & 100 & 0 & 0 & 0 \\
\hline 8 & $\mathrm{Et}_{3} \mathrm{~N}(1)$ & $4 \mathrm{~min}$ & 0 & 80 & 20 & \\
\hline 9 & $\mathrm{Et}_{3} \mathrm{~N}(1)$ & $55 \mathrm{~min}$ & 0 & 79 & 21 & \\
\hline 10 & $\mathrm{Et}_{3} \mathrm{~N}(1)$ & 4h $20 \mathrm{~min}$ & 6 & 77 & 16 & \\
\hline 11 & $\mathrm{Et}_{3} \mathrm{~N}(1)$ & $6.5 \mathrm{~h}$ & 17 & 69 & 14 & \\
\hline 12 & $\mathrm{Et}_{3} \mathrm{~N}(1)$ & $24 \mathrm{~h}$ & 37 & 52 & 11 & \\
\hline 13 & $\mathrm{Et}_{3} \mathrm{~N}(1)$ & $80 \mathrm{~h}$ & 83 & 13 & 4 & \\
\hline 14 & $\mathrm{Et}_{3} \mathrm{~N}(1)$ & 7 days & 89 & 8 & 3 & \\
\hline 15 & $\mathrm{Et}_{3} \mathrm{~N}(1)$ & 9 days & 94 & 4 & 2 & \\
\hline 16 & $\mathrm{Et}_{3} \mathrm{~N}(1)$ & 12 days & 98 & 2 & - & \\
\hline 17 & $\mathrm{Et}_{3} \mathrm{~N}(1)$ & 13 days & 100 & - & & \\
\hline 18 & $\mathrm{Et}_{3} \mathrm{~N}(1.2)$ & $3.5 \mathrm{~h}$ & 2 & 77 & 21 & \\
\hline
\end{tabular}


+ Representative ${ }^{1} \mathrm{H}$ NMR spectra of monitorization of synthesis of 3ab under different conditions.

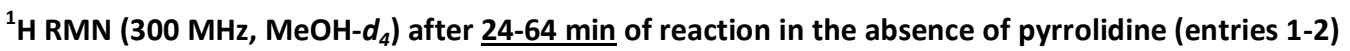

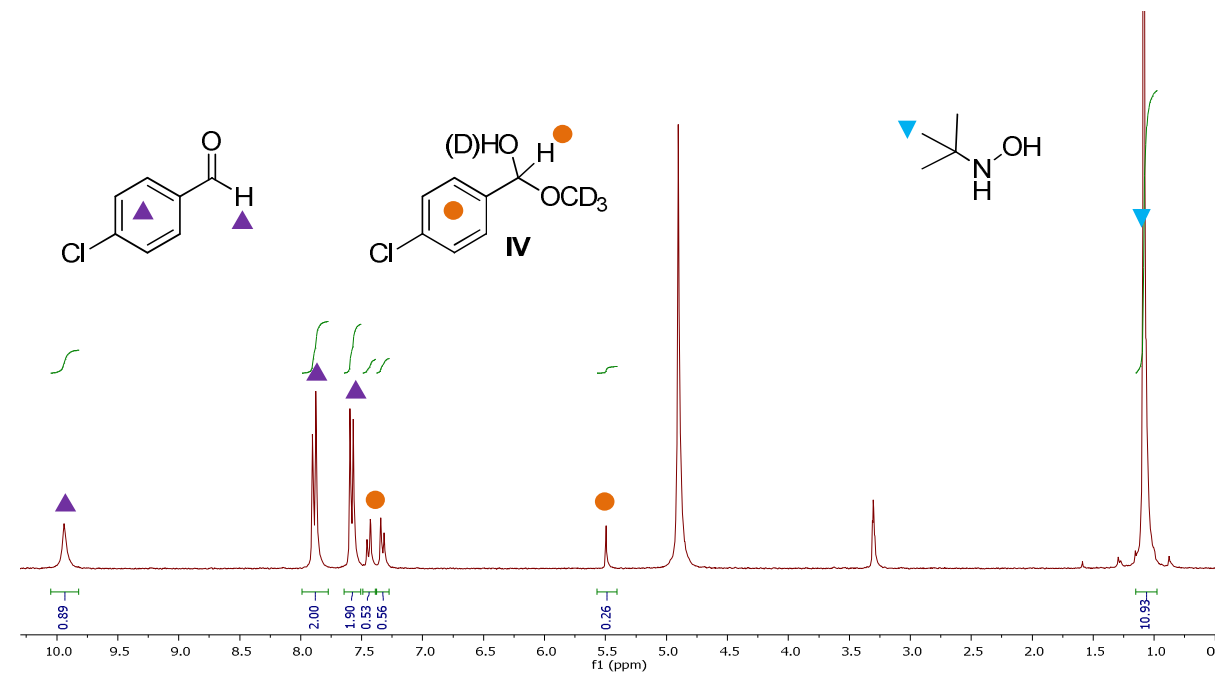

${ }^{1} \mathrm{H} \mathrm{RMN} \mathrm{(300} \mathrm{MHz}, \mathrm{MeOH}-d_{4}$ ) after $24 \mathrm{~min}$ of reaction using pyrrolidine (20 mol\%) (entry 3)

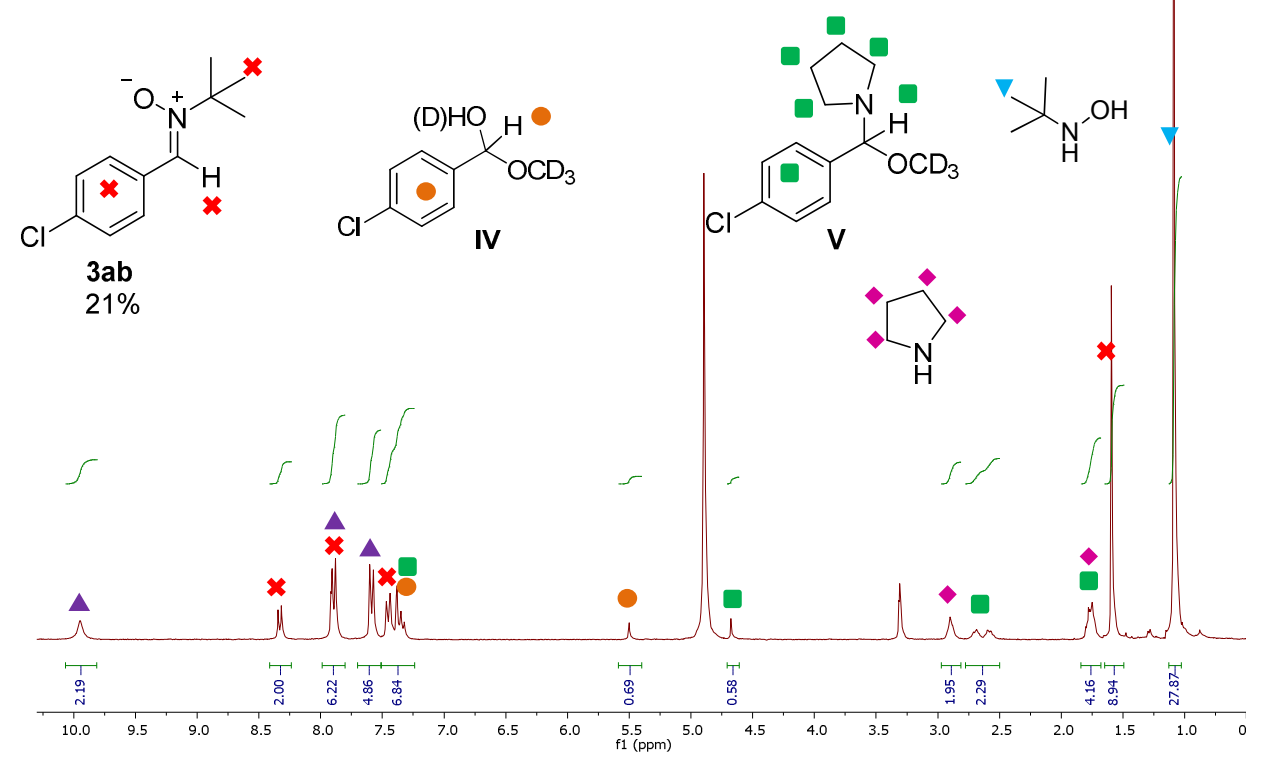


${ }^{1} \mathrm{H}$ RMN (300 MHz, MeOH- $d_{4}$ ) after 64 min of reaction using pyrrolidine (20 mol\%) (entry 4)
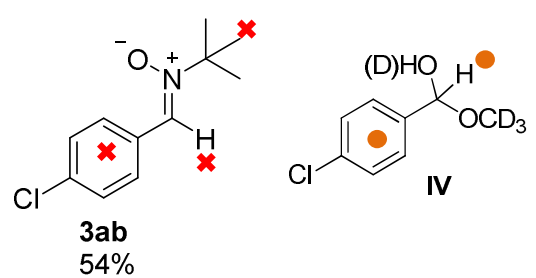

$54 \%$

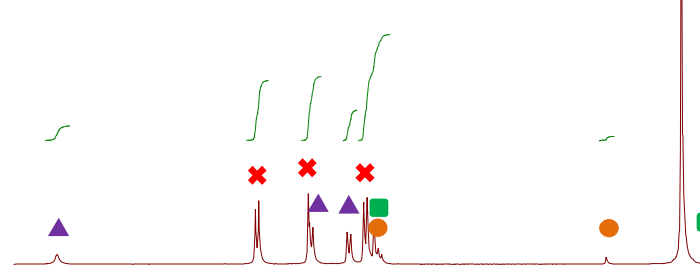

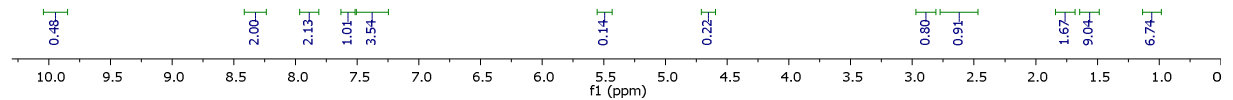

${ }^{1} \mathrm{H}$ RMN (300 MHz, MeOH- $d_{4}$ ) after $\underline{4 \mathrm{~min}}$ of reaction using con $2 \mathrm{~b} \cdot \mathrm{HCl}$ and pyrrolidine (1.2 equiv) (entry 6)

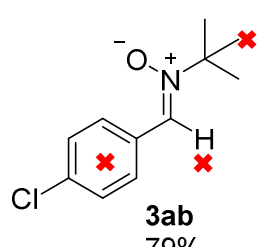

$79 \%$

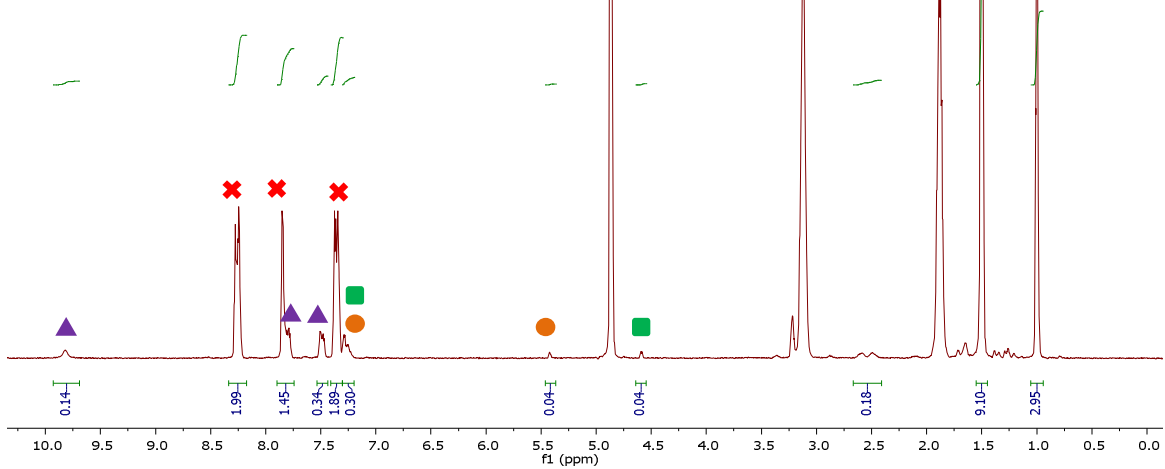


${ }^{1} \mathrm{H} \mathrm{RMN} \mathrm{(300} \mathrm{MHz,} \mathrm{CD}_{2} \mathrm{Cl}_{2}$ ) after $\underline{4 \mathrm{~min}}$ of reaction using con $2 \mathrm{~b} \cdot \mathrm{HCl}$ and pyrrolidine (1.2 equiv) (entry 10)
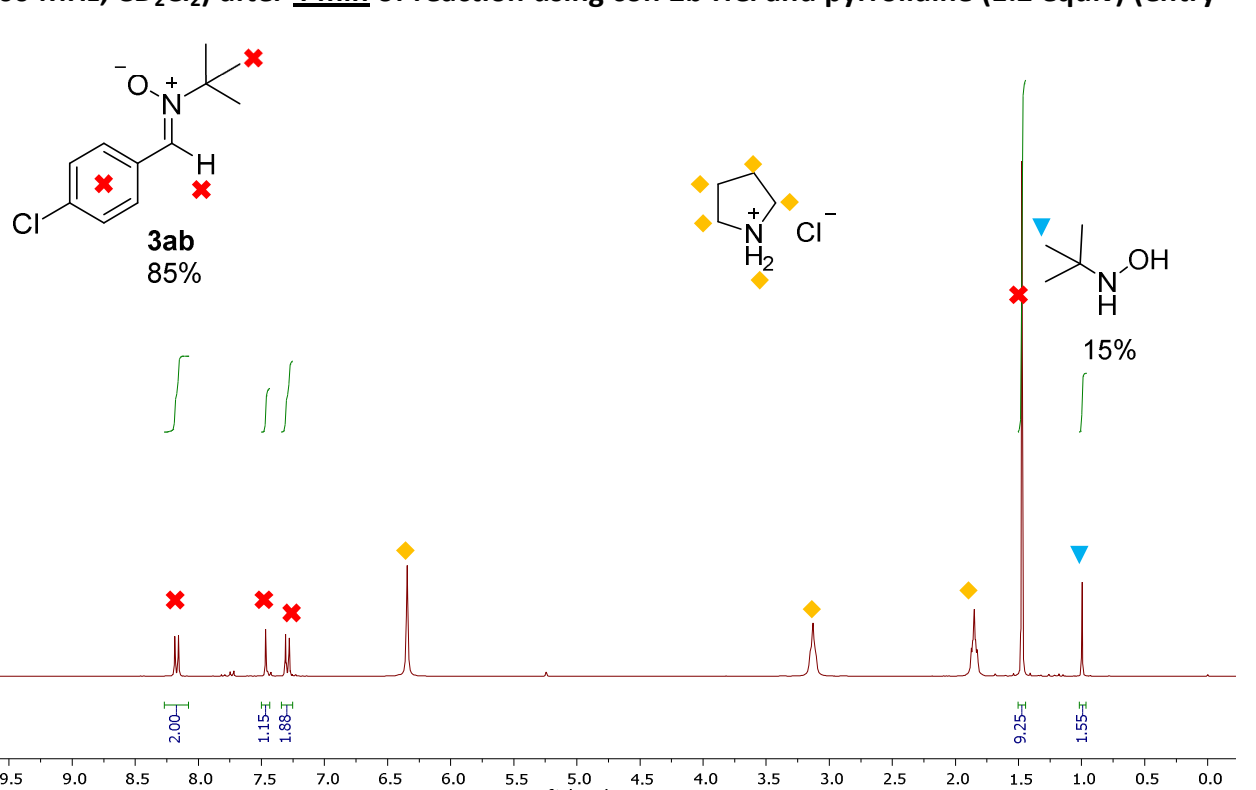

$\left.{ }^{1} \mathrm{H} \mathrm{RMN} \mathrm{(300} \mathrm{MHz}, \mathrm{CD}_{2} \mathrm{Cl}_{2}\right)$ after $\underline{\mathbf{2} \min }$ of reaction using con $2 \mathrm{~b} \cdot \mathrm{HCl}$ and pyrrolidine (2 equiv) (entry 11)

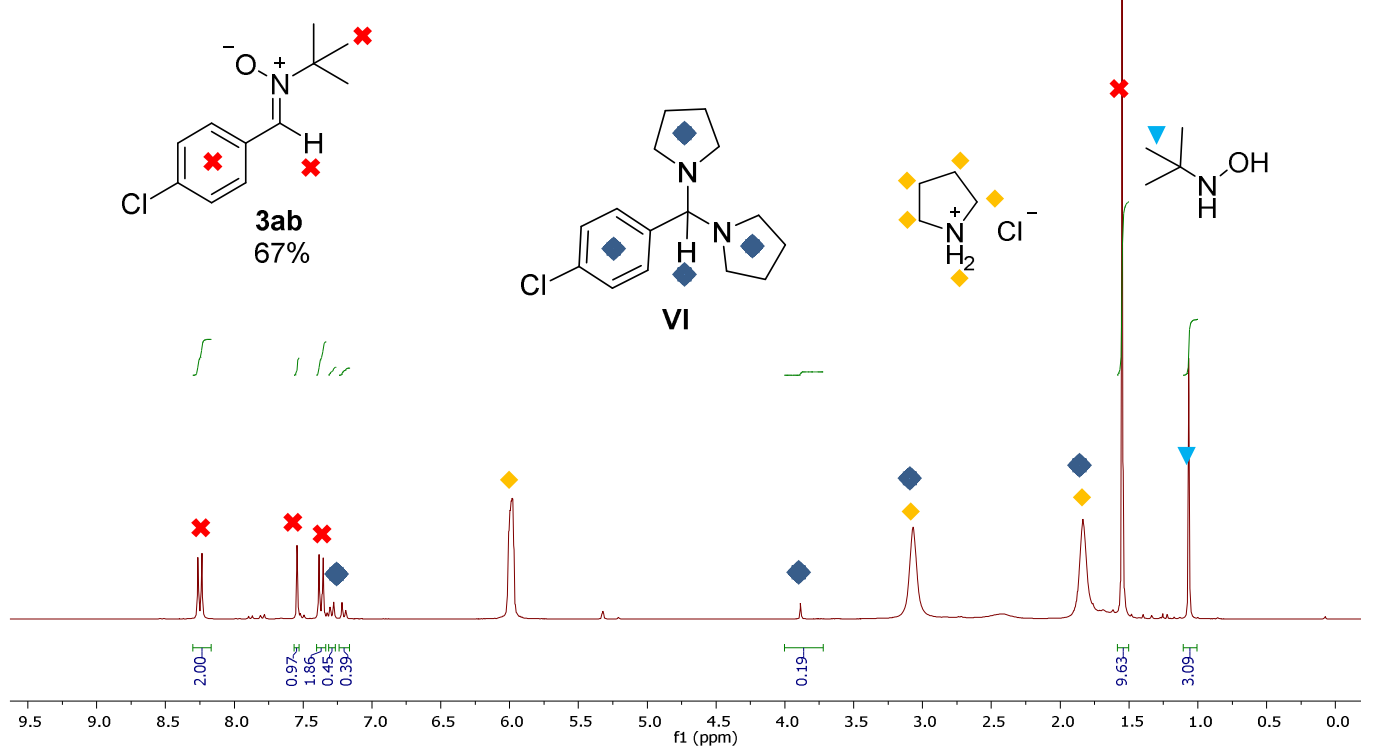


We have characterized hemiacetal IV and hemiaminal $\mathbf{V}$ as they were also formed by treating aldehyde 1a with $\mathrm{MeOH}-d_{4}$ and treating aldehyde 1a with pyrrolidine in $\mathrm{MeOH}-d_{4}$, respectively (see ${ }^{1} \mathrm{H}$ RMN spectra below).
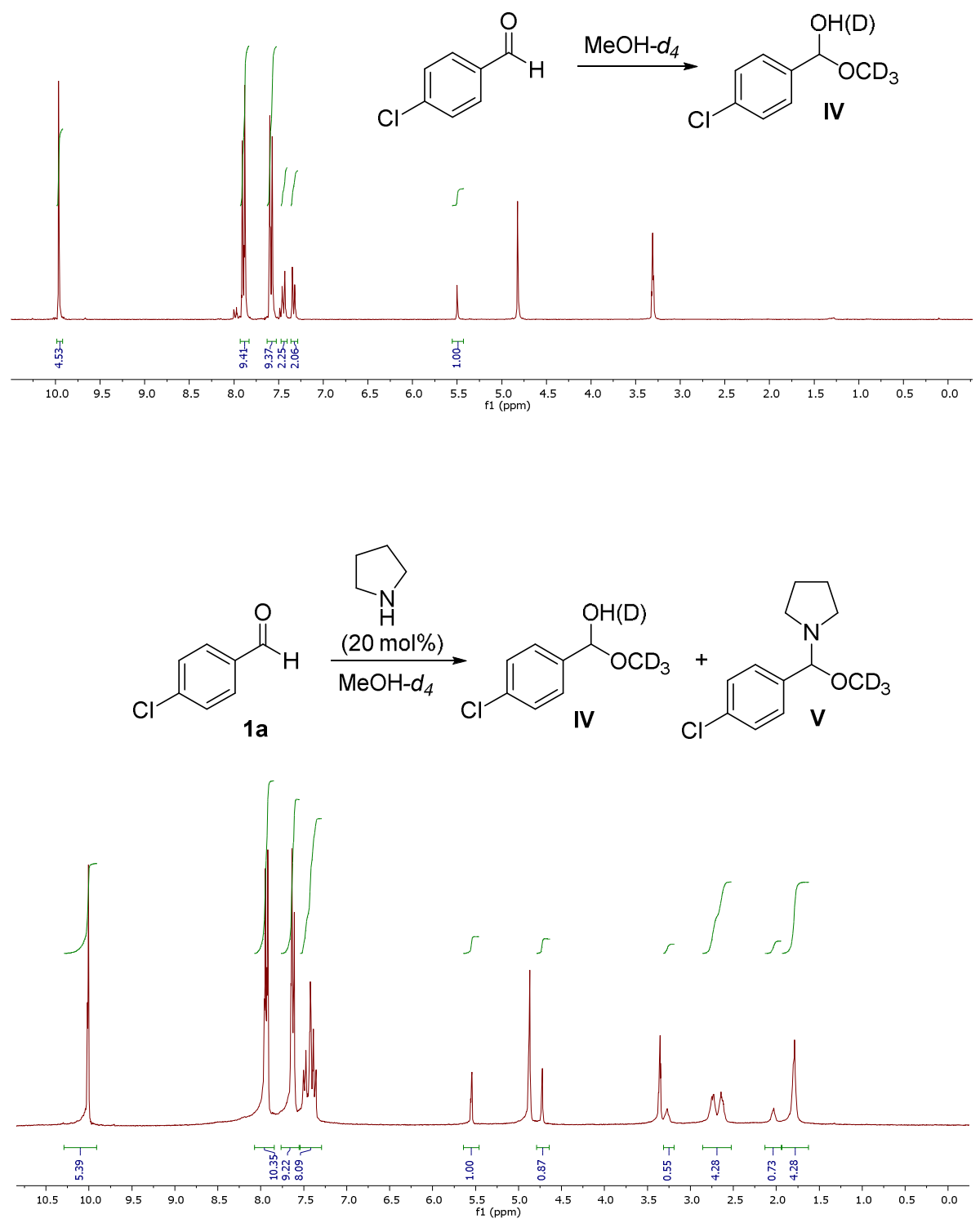
+ Monitorization of the evolution of iminium ion 4 in the presence of $2 \mathrm{~b} \cdot \mathrm{HCl}$.

Preparation of the pyrrolidinium salt of p-chlorobenzaldehyde 4

1-(4-chlorobenzylidene)pyrrolidin-1-ium chloride 4 was prepared using the described procedure for the phenyl derivative under slight modifications. ${ }^{17}$

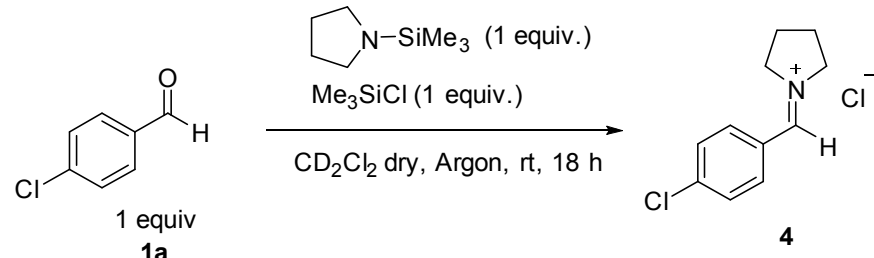

To a solution of $p$-chlorobenzaldehyde ${ }^{18} 1 \mathrm{a}(14.5 \mathrm{mg}, 0.103 \mathrm{mmol})$ in $0.4 \mathrm{~mL}$ of $\mathrm{CD}_{2} \mathrm{Cl}_{2}$ under an argon atmosphere in a NMR tube, trimethylsilyl pyrrolidine $(18 \mu \mathrm{L}, 0.103 \mathrm{mmol})$ and trimethylchlorosilane $(13 \mu \mathrm{L}, 0.103 \mathrm{mmol})$ were successively added. After $20 \mathrm{~h}$, a mixture of 1-(4chlorobenzylidene)pyrrolidin-1-ium chloride 4, p-chorobenzaldehyde $1 \mathbf{1 a}$ and pyrrolidinium chloride was obtained (see ${ }^{1} \mathrm{H}$ NMR below). This mixture was directly used in the following reaction.

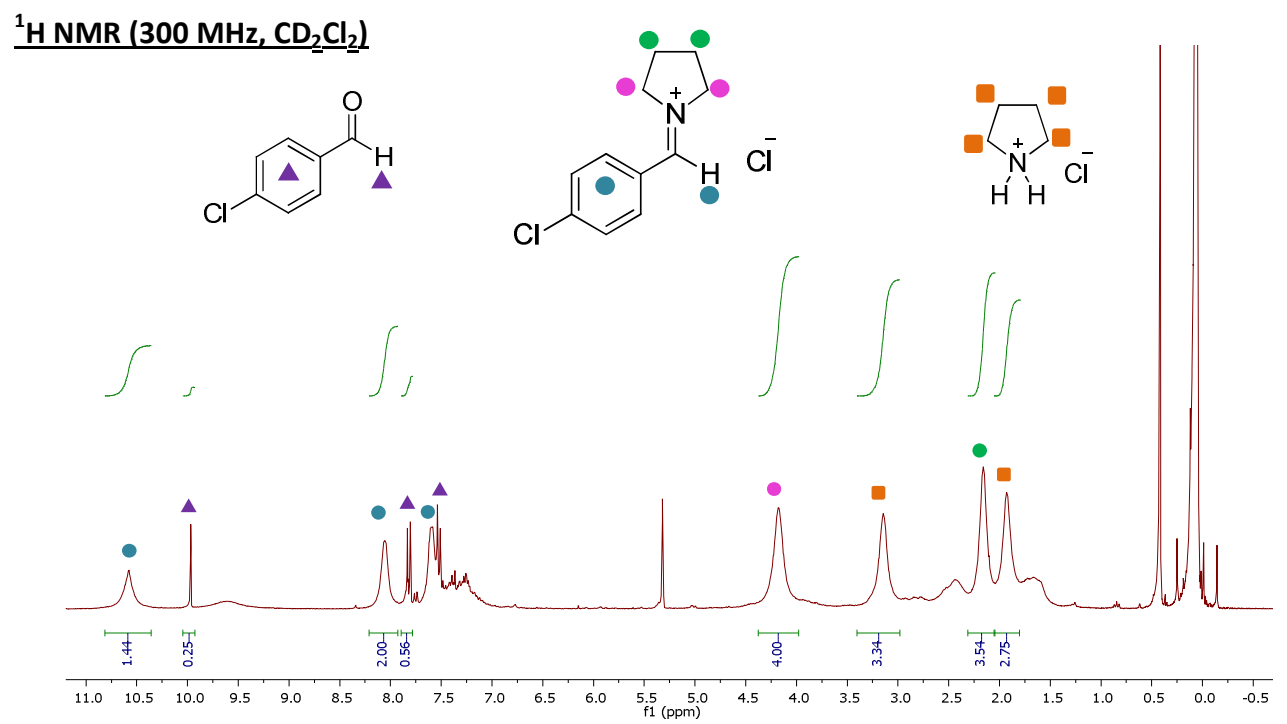


Reaction of pyrrolidinium salt of $p$-chlorobenzaldehyde 4 with $N$-t-butylhydroxylamine hydrochloride $\mathbf{2 b} \cdot \mathbf{H C l}$

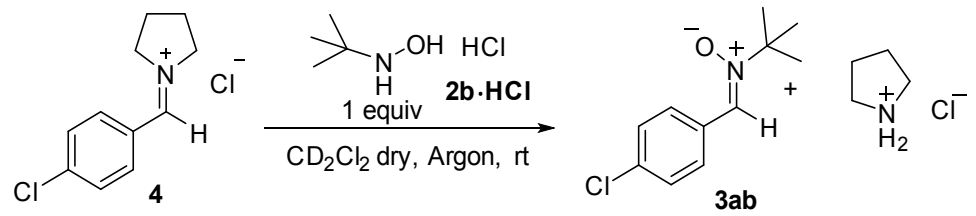

$12.9 \mathrm{mg}$ of $\mathrm{N}$-t-butylhydroxylamine hydrochloride $\mathbf{2 b} \cdot \mathbf{H C l}(0.103 \mathrm{mmol})$ solved in $0.4 \mathrm{~mL}$ of $\mathrm{CD}_{2} \mathrm{Cl}_{2}$ were added to the previous reaction carried out on the NMR tube under inert conditions. We could detect the instantaneous formation of the corresponding nitrone 3ab, result of the attack of $\mathbf{2} \mathbf{b} \cdot \mathbf{H C l}$ to the iminium ion and an increase of the leaving pyrrolidinium chloride $\left(\right.$ see ${ }^{1} \mathrm{H}$ NMR below). It is worth noting that the formation of nitrone is not completed due to the difficulties in the addition of $\mathbf{2} \mathbf{b} \cdot \mathbf{H C l}$ in $\mathrm{CD}_{2} \mathrm{Cl}_{2}$ to the NMR tube.

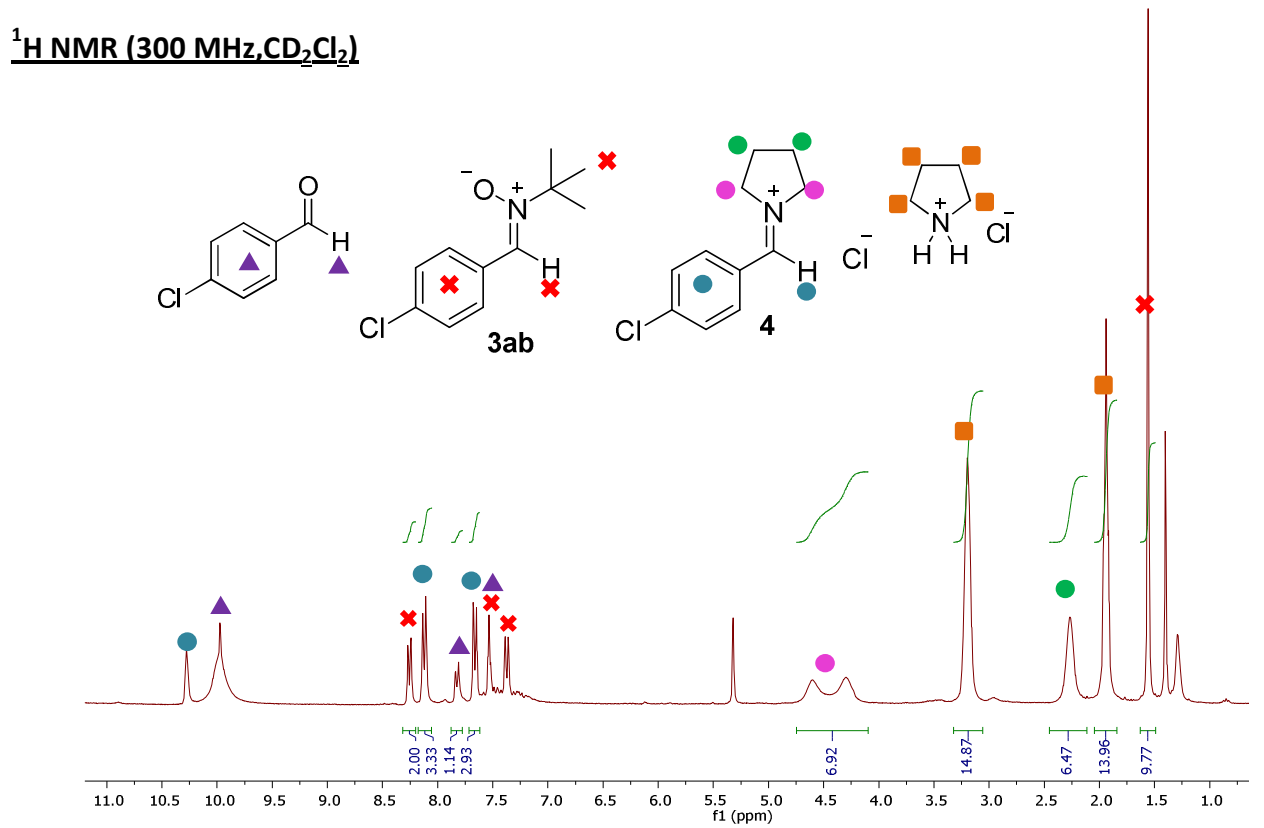




\section{EXPERIMENTAL SECTION}

\section{+ General remarks}

NMR spectra were acquired using $C \mathrm{CDCl}_{3}, \mathrm{MeOH}-d_{4}, \mathrm{CD}_{2} \mathrm{Cl}_{2}$ and $\mathrm{D}_{2} \mathrm{O}$ as solvents, running at 300 and $75 \mathrm{MHz}$ for ${ }^{1} \mathrm{H}$ and ${ }^{13} \mathrm{C}$ respectively. Chemical shifts $(\delta)$ are reported in ppm relative to residual solvent signals $\left(\mathrm{CHCl}_{3}, 7.26 \mathrm{ppm}\right.$ for ${ }^{1} \mathrm{H} \mathrm{NMR} ; \mathrm{CDCl}_{3}, 77.0 \mathrm{ppm}$ for ${ }^{13} \mathrm{C} \mathrm{NMR} ; \mathrm{MeOH}, 3.31$ ppm for ${ }^{1} \mathrm{H}$ NMR; $\mathrm{CH}_{2} \mathrm{Cl}_{2}, 5.32 \mathrm{ppm}$ and $\mathrm{H}_{2} \mathrm{O}, 4.79$ ppm for ${ }^{1} \mathrm{H}$ NMR). In all ${ }^{1} \mathrm{H}$ NMR spectra, multiplicity is indicated as follows: bs (broad singlet), s (singlet), $d$ (doublet), $t$ (triplet), $q$ (quartet) or $\mathrm{m}$ (multiplet). Coupling constant values (in Hertz) and number of protons for each signal are also indicated.

Melting points were measured using Büchi B-540 apparatus in open capillary vials.

For thin layer chromatography (TLC), Supelco silica gel plates with fluorescence indicator 254 $\mathrm{nm}$ were used and compounds were visualized by irradiation with UV light and/or by treatment with a solution of phosphomolybdic acid in EtOH followed by heating.

Silica gel pore $60 \AA, 40-63 \mu \mathrm{m}$ and $\mathrm{Al}_{2} \mathrm{O}_{3}$ (basic brockmann I, standard grade, ca. 150 mesh) were used for filtration.

Mass spectra were obtained in a VG AutoSpec Spectrometer in positive electrospray ionisation (ESI+). Obtained data are expressed in mass/charge $(\mathrm{m} / \mathrm{z})$ units. Values between parentheses indicate relative intensities with regard to the base peak.

All solvent were used without previous treatment. EtOAc was used in analytical grade and dichloromethane stabilized with approx. 50ppm of amylene. Although we have proven that tap water can be used, the results presented herein have been obtained using deionized water.

All starting materials were purchased from commercial suppliers. Aldehydes were washed with aqueous saturated $\mathrm{NaHCO}_{3}$ to remove acids traces. Pyrrolidine $>99.5 \%$ pure was used. 
+ Synthesis of nitrones: general procedures

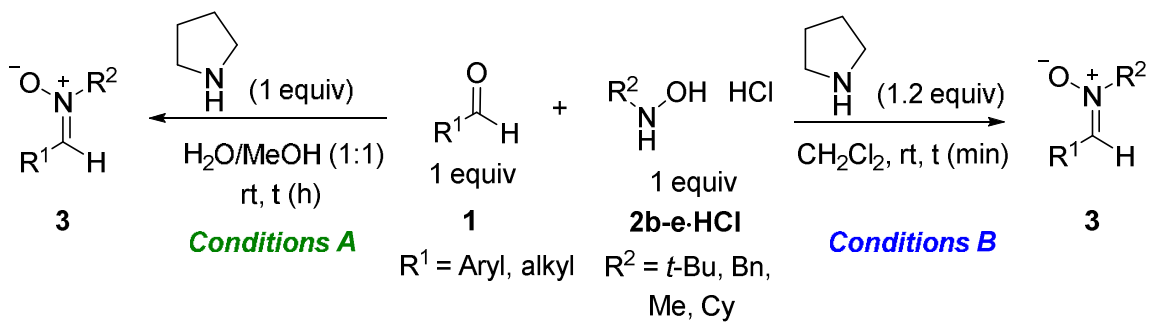

Conditions A: To a $0.33 \mathrm{M}$ solution of an equimolar amount of $\mathrm{N}$-hydroxylamine hydrochloride $\mathbf{2 b}$-e $\cdot \mathbf{H C l}(0.2 \mathrm{mmol})$ and the corresponding aldehyde (1) in $\mathrm{H}_{2} \mathrm{O} / \mathrm{MeOH}(1: 1)$, pyrrolidine (16.7 $\mu \mathrm{L}, 0.2 \mathrm{mmol}, 1$ equiv) was added.

Conditions B: To a $0.33 \mathrm{M}$ solution of an equimolar amount of $\mathrm{N}$-hydroxylamine hydrochloride $\mathbf{2 b}$-e $\cdot \mathbf{H C l}(0.2 \mathrm{mmol})$ and the corresponding aldehyde (1) in $\mathrm{CH}_{2} \mathrm{Cl}_{2}$, pyrrolidine (20 $\mu \mathrm{L}, 0.24 \mathrm{mmol}, 1.2$ equiv) was added. ${ }^{19}$

For both conditions, the mixture was stirred at room temperature during the time indicated in each case. Then, the reaction was filtered through a short pad of either silica gel or basic $\mathrm{Al}_{2} \mathrm{O}_{3}$ using a glass frit with $\mathrm{EtOAC}^{20}$ to obtain the final nitrones without any further purification.

It is worth noting that pyrrolidinium chloride stays trapped in either silica gel or in basic $\mathrm{Al}_{2} \mathrm{O}_{3}$ during filtration. Silica gel was used for filtration of aromatic nitrones and basic $\mathrm{Al}_{2} \mathrm{O}_{3}$ was used for the aliphatic and $\alpha, \beta$-insaturated ones. Unless otherwise stated, the following nitrones were prepared according with general methods and characterized by comparison of their spectroscopic data with those reported.

\section{(Z)-N-(4-Chlorobenzylidene)-2-methylpropan-2-amine oxide (3ab) ${ }^{21}$}<smiles>CC(C)(C)[N+]([O-])=Cc1ccc(Cl)cc1</smiles>

Conditions A: Reaction time: 16h. Yield: 94\%.

Nitrone 3ab was scaled up to $7.9 \mathrm{mmol}$ of nucleophile $(1 \mathrm{~g})$ at $0.66 \mathrm{M}$ under conditions $\mathbf{A}$. The reaction was stirred overnight at room temperature, whereupon water $(5 \mathrm{~mL})$ was added and then extracted with EtOAc $(3 \times 15 \mathrm{~mL})$ and dried over $\mathrm{MgSO}_{4}$. After removal of the solvent under reduced pressure, nitrone 3ab was obtained without purification in $92 \%$ isolated yield.

Conditions B: Reaction time: 20 min. Yield: 98\%

${ }^{1} \mathrm{H}$ NMR $\left(300 \mathrm{MHz}, \mathrm{CDCl}_{3}\right) \delta 8.25(\mathrm{~d}, J=8.6 \mathrm{~Hz}, 2 \mathrm{H}), 7.53(\mathrm{~s}, 1 \mathrm{H}), 7.38(\mathrm{~d}, J=8.6 \mathrm{~Hz}, 2 \mathrm{H}), 1.61(\mathrm{~s}$, $9 \mathrm{H})$. 
(Z)-N-Benzylidene-2-methylpropan-2-amine oxide (3bb) ${ }^{21}$<smiles>CC(C)(C)[N+](=O)/C=C1/C=CC=CC1</smiles>

Conditions A: Reaction time: 6 h. Yield: 91\%

Conditions B: Reaction time: 15 min. Yield: 97\%

${ }^{1} \mathrm{H}$ NMR $\left(300 \mathrm{MHz}, \mathrm{CDCl}_{3}\right) \delta 8.29(\mathrm{~d}, J=7.6,2 \mathrm{H}), 7.55(\mathrm{~s}, 1 \mathrm{H}), 7.35-7.47(\mathrm{~m}, 3 \mathrm{H})$,

$1.62(\mathrm{~s}, 9 \mathrm{H})$.

\section{(Z)-N-(4-Methoxybenzylidene)-2-methylpropan-2-amine oxide (3cb) ${ }^{21}$}

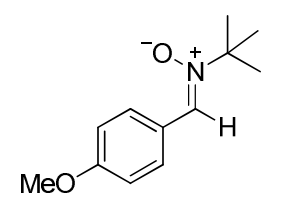

Conditions A: Reaction was carried out with 1.1 equivalents of pyrrolidine in $\mathrm{MeOH}$. Reaction time: 8h. Yield: 90\%. Alternatively, we carried out the reaction using 1 equiv of pyrrolidine in $\mathrm{MeOH}$, obtaining quantitative isolated yield after $21 \mathrm{~h}$.

Conditions B: Reaction time: 45 min. Yield: $94 \%$

${ }^{1} \mathrm{H}$ NMR $\left(300 \mathrm{MHz}, \mathrm{CDCl}_{3}\right) \delta 8.28(\mathrm{~d}, J=8.8 \mathrm{~Hz}, 2 \mathrm{H}), 7.47(\mathrm{~s}, 1 \mathrm{H}), 6.93(\mathrm{~d}, J=8.8 \mathrm{~Hz}, 2 \mathrm{H}), 3.85(\mathrm{~s}$, $3 \mathrm{H}), 1.61(\mathrm{~s}, 9 \mathrm{H})$.

(Z)-N-(4-Nitrobenzylidene)-2-methyl-propan-2-amine oxide (3db) $)^{21}$

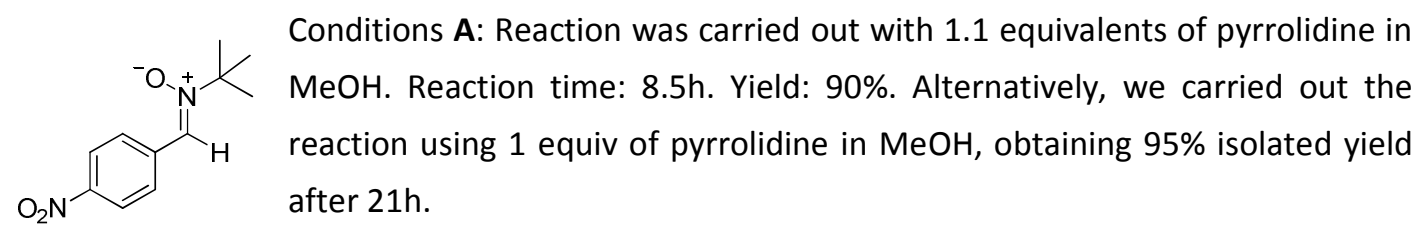

Conditions B: Reaction time: 34 min. Yield: 100\%

${ }^{1} \mathrm{H}$ NMR $\left(300 \mathrm{MHz}, \mathrm{CDCl}_{3}\right) \delta 8.45(\mathrm{~d}, J=9.0 \mathrm{~Hz}, 2 \mathrm{H}), 8.25(\mathrm{~d}, J=9.1 \mathrm{~Hz}, 2 \mathrm{H}), 7.71(\mathrm{~s}, 1 \mathrm{H}), 1.64(\mathrm{~s}$, $9 \mathrm{H})$.

(Z)-N-(2-Chlorobenzylidene)-2-methylpropan-2-amine oxide (3eb)

Conditions A: Reaction time: $16 \mathrm{~h}$. Quantitative yield.
Conditions B: Reaction time: 20 min. Quantitative yield. $7.34(\mathrm{~d}, \mathrm{~J}=7.4 \mathrm{~Hz}, 1 \mathrm{H}), 7.30-7.17(\mathrm{~m}, 2 \mathrm{H}), 1.56(\mathrm{~s}, 9 \mathrm{H})$.

${ }^{13} \mathrm{C}$ NMR $\left(75 \mathrm{MHz}, \mathrm{CDCl}_{3}\right) \delta 133.3(\mathrm{CH}=\mathrm{N}), 130.7(\mathrm{CH}), 129.3(\mathrm{CH}), 129.1(\mathrm{CH}), 128.6(\mathrm{C}), 127.0$ $(\mathrm{CH}), 125.9(\mathrm{C}), 71.9(\mathrm{C}), 28.3\left(\mathrm{CH}_{3}\right)$.

MS (ESI+): m/z $212\left(\mathrm{M}^{+}+1\right)(82), 158$ (25), $156(100)$. HRMS (ESI+): calculated for $\mathrm{C}_{11} \mathrm{H}_{15} \mathrm{CINO}$ $\left(M^{+}+1\right)$ : 212.0836; found: 212.0843 . 
(Z)-N-(2-Bromobenzylidene)-2-methylpropan-2-amine oxide (3fb) ${ }^{2}$

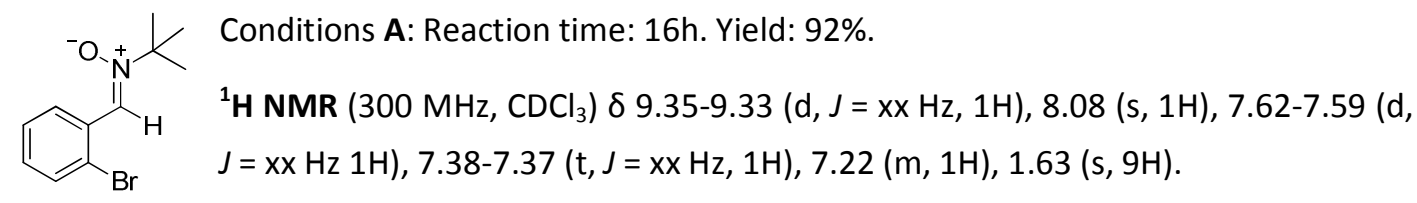

(Z)-N-(2-Methoxybenzylidene)-2-methylpropan-2-amine oxide (3gb) $)^{22}$

Conditions A: Reaction time: 16h. Quantitative yield.
${ }^{1} \mathrm{H} \mathrm{NMR}\left(300 \mathrm{MHz}, \mathrm{CDCl}_{3}\right): \delta 9.36(\mathrm{~d}, J=7.8 \mathrm{~Hz}, 1 \mathrm{H}), 8.05(\mathrm{~s}, 1 \mathrm{H}), 7.35-7.33(\mathrm{~m}, 1$ H), 7.04-6.86 (m, 2 H), $3.87(\mathrm{~s}, 3 \mathrm{H}), 1.61(\mathrm{~s}, 9 \mathrm{H})$.

(Z)-N-(2-hydroxybenzylidene)-2-methylpropan-2-amine oxide (3hb $)^{23}$

Conditions B: Reaction time: 1 min. Quantitative yield
${ }_{\mathrm{H}} \mathrm{H} \mathrm{NMR}\left(300 \mathrm{MHz}, \mathrm{CD}_{2} \mathrm{Cl}_{2}\right) \delta=7.77(\mathrm{~s}, 1 \mathrm{H}), 7.39(\mathrm{t}, J=7.3 \mathrm{~Hz}, 1 \mathrm{H}), 7.18(\mathrm{~d}, J=7.7$

(Z)-N-(2-Hydroxy-3-methoxybenzylidene)-2-methylpropan-2-amine oxide (3ib)

Conditions A: Reaction time: 8h. Quantitative yield.
Conditions B: Reaction time: 9 min. Quantitative yield. $9 \mathrm{H})$.

${ }^{13} \mathrm{C} \mathrm{NMR}\left(75 \mathrm{MHz}, \mathrm{CDCl}_{3}\right) \delta 150.8$ (C), 150.3 (C), $137.2(\mathrm{CH}=\mathrm{N}), 123.5(\mathrm{CH}), 118.5(\mathrm{CH}), 117.3(\mathrm{C})$, $114.6(\mathrm{CH}), 70.1(\mathrm{C}), 56.3\left(\mathrm{CH}_{3}\right), 28.3\left(3 \times \mathrm{CH}_{3}\right)$.

MS (ESI+): m/z $224\left(M^{+}+1\right)(100), 168$ (58). HRMS (ESI+): calculated for $\mathrm{C}_{12} \mathrm{H}_{18} \mathrm{NO}_{3}\left(\mathrm{M}^{+}+1\right)$ : 224.1281; found: 224.1290 . 
(Z)-N-(2-hydroxy-4-methoxybenzylidene)-2-methylpropan-2-amine oxide (3jb) ${ }^{1}$

Conditions A: Reaction time: 16h. Quantitative yield.
${ }^{1} \mathrm{H}$ NMR $\left(300 \mathrm{MHz} C D C l_{3}\right) \delta 7.53(\mathrm{~s}, 1 \mathrm{H}), 6.94(\mathrm{~d}, J=8.6 \mathrm{~Hz}, 1 \mathrm{H}), 6.43-6.37(\mathrm{~m}$, $2 \mathrm{H}), 3.77(\mathrm{~s}, 3 \mathrm{H}), 1.58(\mathrm{~s}, 9 \mathrm{H})$.

(Z)-N-(2-Hydroxy-5-methylbenzylidene)-2-methylpropan-2-amine oxide (3kb)

Yellow solid. m.p. $132-134^{\circ} \mathrm{C} .{ }^{1} \mathrm{H} N \mathrm{NMR}\left(300 \mathrm{MHz}, \mathrm{CDCl}_{3}\right) \delta 7.66(\mathrm{~s}, 1 \mathrm{H}), 7.17(\mathrm{~d}, J$
$=8.3 \mathrm{~Hz}, 1 \mathrm{H}), 6.93-6.77(\mathrm{~m}, 2 \mathrm{H}), 2.24(\mathrm{~s}, 3 \mathrm{H}), 1.61(\mathrm{~s}, 9 \mathrm{H})$.

${ }^{13}$ C NMR (75 MHz, CDCl $)$ ) 157.4 (C), 136.9 (CH=N), $134.5(\mathrm{CH}), 131.9(\mathrm{CH}), 127.8(\mathrm{C}), 119.9(\mathrm{CH})$, 116.7 (C), 69.9 (C), $28.3\left(3 \times \mathrm{CH}_{3}\right), 20.1\left(\mathrm{CH}_{3}\right)$.

MS (ESI+): m/z $208\left(M^{+}+1\right)(100), 152(46)$. HRMS (ESI+): calculated for $\mathrm{C}_{12} \mathrm{H}_{18} \mathrm{NO}_{2}\left(\mathrm{M}^{+}+1\right)$ : 208.1332; found: 208.1337.

(Z)-N-(5-Chloro-2-hydroxybenzylidene)-2-methylpropan-2-amine oxide (3lb)<smiles>CC(C)(C)N=Cc1cc(Cl)ccc1O</smiles>

Conditions A: Reaction time: $6 \mathrm{~h}$. Yield: $98 \%$.

Yellow solid. m.p. $138-140^{\circ} \mathrm{C} .{ }^{1} \mathrm{H}$ NMR $\left(300 \mathrm{MHz}, \mathrm{CDCl}_{3}\right) \delta 7.58(\mathrm{~s}, 1 \mathrm{H}), 7.22$ (dd, $J=8.9 \mathrm{~Hz}, 2.6 \mathrm{~Hz}, 1 \mathrm{H}), 6.99(\mathrm{~d}, J=2.6 \mathrm{~Hz}, 1 \mathrm{H}), 6.82(\mathrm{~d}, J=8.9 \mathrm{~Hz}, 1 \mathrm{H}), 1.55$ (s, $9 \mathrm{H})$.

${ }^{13} \mathrm{C} \mathrm{NMR}\left(75 \mathrm{MHz}, \mathrm{CDCl}_{3}\right) \delta 158.4(\mathrm{C}), 135.9(\mathrm{CH}=\mathrm{N}), 133.2(\mathrm{CH}), 130.9(\mathrm{CH}), 123.4(\mathrm{C}), 121.6(\mathrm{CH})$, 118.3 (C), 70.6 (C), $28.3\left(3 \times \mathrm{CH}_{3}\right)$.

MS (ESI+): m/z $228\left(M^{+}+1\right)$ (100), 174 (12), 172 (51). HRMS (ESI+): calculated for $\mathrm{C}_{11} \mathrm{H}_{15} \mathrm{CINO}_{2}$ $\left(M^{+}+1\right): 228.0785$; found: 228.0776 .

(Z)-N-(2-Hydroxy-5-nitrobenzylidene)-2-methylpropan-2-amine oxide (3mb) ${ }^{1}$<smiles></smiles>

Conditions A: Reaction time: $6.5 \mathrm{~h}$. Yield: $96 \%$.

${ }^{1} \mathrm{H}$ NMR $\left(300 \mathrm{MHz}, \mathrm{CDCl}_{3}\right) \delta=8.17(\mathrm{~d}, J=9.2 \mathrm{~Hz}, 1 \mathrm{H}), 8.10(\mathrm{~s}, 1 \mathrm{H}), 7.78(\mathrm{~s}, 1 \mathrm{H})$, $6.92(\mathrm{~d}, J=9.1 \mathrm{~Hz}, 1 \mathrm{H}), 1.64(\mathrm{~s}, 9 \mathrm{H})$. 


\section{(Z)-N-(Pyridin-2-ylmethylene)-2-methylpropan-2-amine oxide (3nb) ${ }^{7}$}<smiles>CC(C)(C)[N+](=O)/C=N/c1ccccn1</smiles>

Conditions A: Reaction time: 7h. Yield: 96\%.

Conditions B: Reaction time: 8 min. Yield: $100 \%$.

${ }^{1}$ H NMR $\left(300 \mathrm{MHz}, \mathrm{CDCl}_{3}\right) \delta 9.22(\mathrm{~d}, J=8.1 \mathrm{~Hz}, 1 \mathrm{H}), 8.65-8.61(\mathrm{~m}, 1 \mathrm{H}), 7.92(\mathrm{~s}$, $1 \mathrm{H}), 7.79(\mathrm{t}, J=7.7 \mathrm{~Hz}, 1 \mathrm{H}), 7.30-7.26(\mathrm{~m}, 1 \mathrm{H}), 1.64(\mathrm{~s}, 9 \mathrm{H})$.

\section{(Z)-N-((2-Chloropyridin-3-yl)methylene)-2-methylpropan-2-amine oxide (3ob) ${ }^{10}$}<smiles>CC(C)(C)/N=C/c1cccnc1Cl</smiles>

Conditions A: Reaction time: $16 \mathrm{~h}$. Yield: $92 \%$.

${ }^{1} \mathbf{H}$ NMR $\left(300 \mathrm{MHz}, \mathrm{CDCl}_{3}\right) \delta 9.67(\mathrm{~d}, J=7.9 \mathrm{~Hz}, 1 \mathrm{H}), 8.30(\mathrm{~d}, J=4.2 \mathrm{~Hz}, 1 \mathrm{H}), 8.05$ (s, $1 \mathrm{H}), 7.29$ (dd, $J=7.9,4.2 \mathrm{~Hz}, 1 \mathrm{H}), 1.61(\mathrm{~s}, 9 \mathrm{H})$.

\section{(Z)-N-((1H-Indol-3-yl)methylene)-2-methylpropan-2-amine oxide (3pb) ${ }^{10}$}

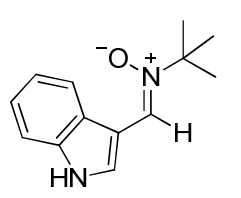

Conditions A: Reaction was carried out with 1.1 equiv of pyrrolidine in $\mathrm{MeOH}$. Reaction time: $29 \mathrm{~h}$. When the crude was filtered through a short pad of silica gel, the product decomposed. Nevertheless, the isolation by extraction with EtOAc $(3 \times 5 \mathrm{~mL})$, dried with $\mathrm{MgSO}_{4}$ and removal of the solvent under vacuum, provided nitrone $\mathbf{3 p b}$ in quantitative yield. The reaction in other solvents (EtOAc and $\mathrm{CH}_{2} \mathrm{Cl}_{2}$ ) were performed following the same procedure above mentioned, obtaining $100 \%$ and $91 \%$ yield respectively after $15 \mathrm{~h}$.

Conditions B: Reaction time: $4 \mathrm{~h}$. The isolation of the product was the same as conditions A. Yield: 92\%.

${ }^{1} \mathrm{H}$ NMR $\left(300 \mathrm{MHz}, \mathrm{CDCl}_{3}\right) \delta 9.01(\mathrm{~d}, J=2.7 \mathrm{~Hz}, 1 \mathrm{H}), 8.98$ (bs, $\left.1 \mathrm{H}\right), 7.98(\mathrm{~s}, 1 \mathrm{H}), 7.67(\mathrm{~d}, J=8.8 \mathrm{~Hz}$, $1 \mathrm{H}), 7.39(\mathrm{~d}, J=8.8 \mathrm{~Hz}, 1 \mathrm{H}), 7.28-7.06(\mathrm{~m}, 2 \mathrm{H}), 1.64(\mathrm{~s}, 9 \mathrm{H})$.

\section{(Z)-N-((E)-3-Phenylallylidene)-2-methylpropan-2-amine oxide (3qb)}

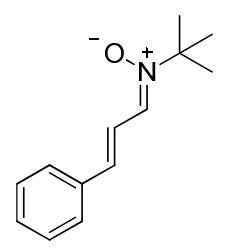

Conditions A: Reaction was carried out in $\mathrm{MeOH}$ as solvent. Reaction time: $4 \mathrm{~h}$. The crude of the reaction was filtered through basic $\mathrm{Al}_{2} \mathrm{O}_{3}$ instead of silica gel. Quantitative yield.

Conditions B: Reaction time: $1 \mathrm{~h}$. The crude of the reaction was filtered through basic $\mathrm{Al}_{2} \mathrm{O}_{3}$. Yield: $98 \%$.

Yellow solid. ${ }^{1} \mathbf{H}$ NMR $\left(300 \mathrm{MHz}, \mathrm{CDCl}_{3}\right) \delta$ 7.53-7.32 (m, 4H), 7.32-7.16 (m, 3H), $6.94(\mathrm{~d}, J=15.9$ $\mathrm{Hz}, 1 \mathrm{H}), 1.65-1.37(\mathrm{~m}, 9 \mathrm{H}) .{ }^{13} \mathrm{C} \mathrm{NMR}\left(75 \mathrm{MHz}, \mathrm{CDCl}_{3}\right) \delta 138.0(\mathrm{CH}=\mathrm{N}), 136.5(\mathrm{CH}), 132.2(\mathrm{C}), 129.0$ (CH), $128.9(2 \times \mathrm{CH}), 127.3(2 \times \mathrm{CH}), 119.6(\mathrm{CH}), 69.4(\mathrm{C}), 28.2\left(3 \times \mathrm{CH}_{3}\right)$. 
MS (ESI+): m/z $204\left(M^{+}+1\right)(100), 148(48)$. HRMS (ESI+): calculated for $\mathrm{C}_{13} \mathrm{H}_{18} \mathrm{NO}\left(\mathrm{M}^{+}+1\right)$ : 204.1382; found: 204.1387.

\section{(Z)-N-(3-Methylbutylidene)-2-methylpropan-2-amine oxide (3sb)}

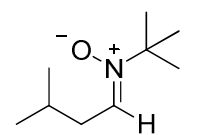

Conditions A: Reaction time: $5.5 \mathrm{~h}$. The crude of the reaction was filtered through basic $\mathrm{Al}_{2} \mathrm{O}_{3}$ instead of silica gel .Yield: $99 \%$.

Conditions B: Reaction time: $3 \mathrm{~min}$. The crude of the reaction was filtered through basic $\mathrm{Al}_{2} \mathrm{O}_{3}$. Yield: 92\%.

${ }^{1} \mathrm{H}$ NMR $\left(300 \mathrm{MHz}, \mathrm{CDCl}_{3}\right) \delta 6.84(\mathrm{t}, J=5.6 \mathrm{~Hz}, 1 \mathrm{H}), 2.55-2.26(\mathrm{~m}, 2 \mathrm{H}), 2.06-1.80(\mathrm{~m}, 1 \mathrm{H}), 1.50$ $(\mathrm{s}, 9 \mathrm{H}), 0.97(\mathrm{~d}, J=6.6 \mathrm{~Hz}, 6 \mathrm{H})$.

\section{(Z)-2-Methyl-N-pentylidenepropan-2-amine- $\mathrm{N}$-oxide (3rb)}

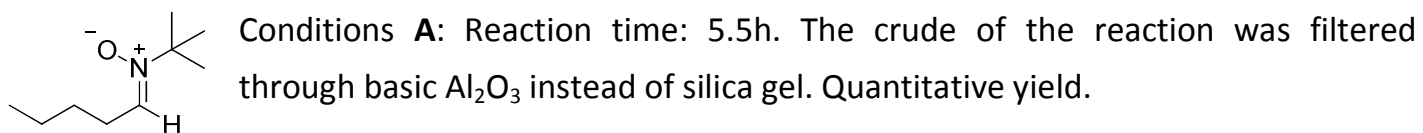

Conditions B: Reaction time: $3 \mathrm{~min}$. The crude of the reaction was filtered through basic $\mathrm{Al}_{2} \mathrm{O}_{3}$. Yield: $100 \%$

Unstable compound. Colourless oil. ${ }^{1} \mathbf{H}$ NMR $\left(300 \mathrm{MHz}, \mathrm{CDCl}_{3}\right) \delta 6.76(\mathrm{t}, J=5.5 \mathrm{~Hz}, 1 \mathrm{H}), 2.55-2.35$ $(\mathrm{m}, 2 \mathrm{H}), 1.60-1.26(\mathrm{~m}, 13 \mathrm{H}), 0.92(\mathrm{t}, J=7.1 \mathrm{~Hz}, 3 \mathrm{H})$.

${ }^{13} \mathrm{C}$ NMR $\left(75 \mathrm{MHz}, \mathrm{CDCl}_{3}\right) \delta 135.0(\mathrm{CH}=\mathrm{N}), 68.9$ (C), $28.1\left(3 \times \mathrm{CH}_{3}\right), 27.9\left(\mathrm{CH}_{2}\right), 26.9\left(\mathrm{CH}_{2}\right), 22.7$ $\left(\mathrm{CH}_{2}\right), 13.9\left(\mathrm{CH}_{3}\right)$.

MS (ESI+): m/z $158\left(\mathrm{M}^{+}+1\right)(100), 180\left(\mathrm{M}^{+}+\mathrm{Na}\right)$ (53), 102 (38). HRMS (ESI+): calculated for $\mathrm{C}_{9} \mathrm{H}_{20} \mathrm{NO}\left(\mathrm{M}^{+}+1\right)$ : 158.1539; found: 158.1541 .

\section{(Z)-N-(Cyclohexylmethylene)-2-methylpropan-2-amine oxide (3tb) ${ }^{24}$}

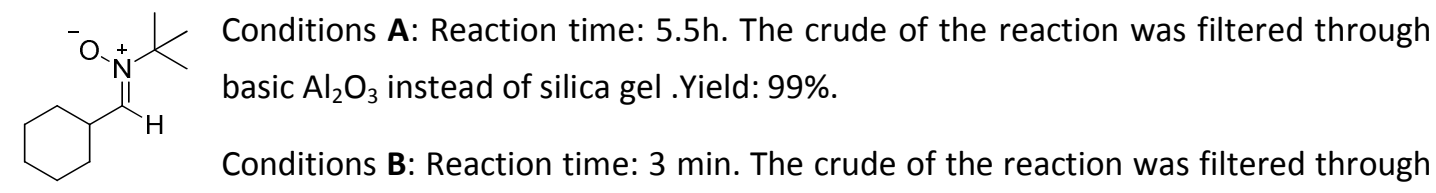
basic $\mathrm{Al}_{2} \mathrm{O}_{3}$. Yield: $98 \%$.

${ }^{1}$ H NMR $\left(300 \mathrm{MHz}, \mathrm{CDCl}_{3}\right) \delta 6.61(\mathrm{~d}, J=7.2 \mathrm{~Hz}, 1 \mathrm{H}), 3.05-2.93(\mathrm{~m}, 1 \mathrm{H}), 1.92-1.85(\mathrm{~m}, 2 \mathrm{H})$, $1.75-1.64(\mathrm{~m}, 3 \mathrm{H}), 1.48(\mathrm{~s}, 9 \mathrm{H}), 1.43-1.33(\mathrm{~m}, 2 \mathrm{H}), 1.30-1.21(\mathrm{~m}, 1 \mathrm{H}), 1.19-1.08(\mathrm{~m}, 2 \mathrm{H})$. 


\section{(Z)-N-(2-Phenylpropylidene)-2-methylpropan-2-amine oxide (3ub) ${ }^{24}$}

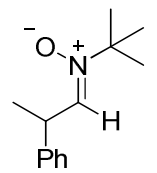

Conditions A: Reaction time: 5.5h. Yield: 96\%.

Conditions B: Reaction time: 12 min. Yield: $80 \%$.

${ }^{1}$ H NMR $\left(300 \mathrm{MHz}, \mathrm{CDCl}_{3}\right) \delta 7.03-7.33(\mathrm{~m}, 5 \mathrm{H}), 6.86(\mathrm{~d}, J=7.5 \mathrm{~Hz}, \mathrm{HC}=\mathrm{N}), 4.35$ (q, $J=$ $7.5 \mathrm{~Hz}, 1 \mathrm{H}), 1.47(\mathrm{~s}, 9 \mathrm{H}), 1.43(\mathrm{~d}, J=5 \mathrm{~Hz}, 3 \mathrm{H})$.

(Z)-N-(4-Chlorobenzylidene)-1-phenylmethanamine oxide (3ac) ${ }^{11}$

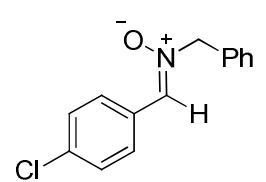

Conditions A: Reaction time: 9h. Yield: 99\%.

Conditions B: Reaction time: 3 min. Yield: 99\%

${ }^{1}$ H-NMR (300 MHz, CDCl $) \delta 8.17(\mathrm{~d}, J=8.8 \mathrm{~Hz}, 2 \mathrm{H}), 7.34-7.49(\mathrm{~m}, 8 \mathrm{H}), 5.05$

$(s, 2 \mathrm{H})$.

(Z)-N-(2,2-Dimethylpropylidene)-1-phenylmethanamine oxide (3vc) ${ }^{25}$

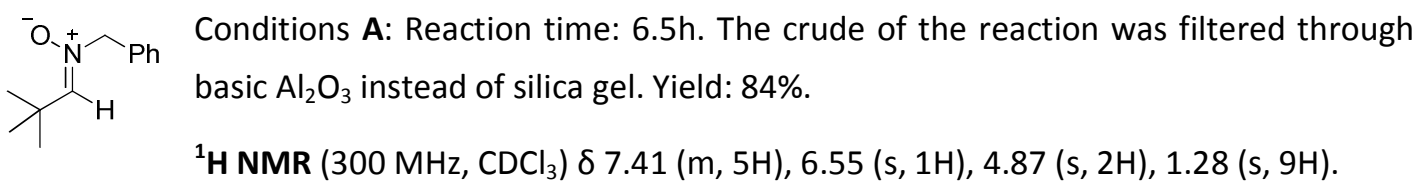

\section{(Z)-N-(4-Chlorobenzylidene)methanamine oxide (3ad) ${ }^{26}$}

Conditions $\mathrm{A}$ : Reaction time: $6 \mathrm{~h}$. The crude of the reaction was filtered through
Under conditions $\mathrm{A}$, we scaled up the formation of nitrone $3 \mathrm{ad}$ to $72 \mathrm{mmol}(\mathbf{6 g}$ of nucleophile) at $0.72 \mathrm{M}$. The reaction was stirred at room temperature during $7 \mathrm{~h}$, whereupon water $(15 \mathrm{~mL})$ was added and the mixture extracted with EtOAc $(3 \times 20 \mathrm{~mL})$ and dried over $\mathrm{MgSO}_{4}$. After removal of the solvent under reduced pressure, nitrone 3ad was obtained without purification in $93 \%$ isolated yield.

Conditions B: Reaction time: $3 \mathrm{~min}$. The crude of the reaction was filtered through basic $\mathrm{Al}_{2} \mathrm{O}_{3}$ instead of silica gel. Yield: 93\%.

Under conditions B, we scaled up the formation of nitrone 3 ad to $14.3 \mathrm{mmol}$ (19 of nucleophile) at $0.66 \mathrm{M}$. The reaction was stirred at room temperature during $15 \mathrm{~min}$. Then, the solvent was evaporated and the crude of reaction was extracted with EtOAc $(4 \times 12 \mathrm{~mL})$ and dried over $\mathrm{MgSO}_{4}$. After removal of the solvent under reduced pressure, nitrone 3ad was obtained without purification in $92 \%$ isolated yield. 
${ }^{1} \mathrm{H}$ NMR $\left(300 \mathrm{MHz}, \mathrm{CDCl}_{3}\right) \delta 8.08(\mathrm{~d}, J=8.5 \mathrm{~Hz}, 2 \mathrm{H}), 7.28(\mathrm{~d}, J=8.5 \mathrm{~Hz}, 2 \mathrm{H}), 7.27(\mathrm{~s}, 1 \mathrm{H}), 3.78(\mathrm{~s}$, $3 \mathrm{H})$.

$(Z)-N-((E)-3-P h e n y l a l l y l i d e n e)$ methanamine oxide (3qd $)^{27}$

- ${ }_{-}^{+}-$Conditions $\mathrm{A}$ : Reaction time: $3 \mathrm{~h}$. The crude of the reaction was filtered through basic $\mathrm{Al}_{2} \mathrm{O}_{3}$ instead of silica gel. Yield: $84 \%$.

Ph ${ }^{1} \mathrm{H}$ NMR $\left(300 \mathrm{MHz}, \mathrm{CDCl}_{3}\right) \delta 7.50(\mathrm{~d}, J=7.4 \mathrm{~Hz}, 2 \mathrm{H}), 7.43(\mathrm{~m}, 1 \mathrm{H}), 7.36-7.28(\mathrm{~m}, 3 \mathrm{H})$, $7.23(\mathrm{~d}, J=9.5 \mathrm{~Hz}, 1 \mathrm{H}), 6.95(\mathrm{~d}, J=16.3 \mathrm{~Hz}, 1 \mathrm{H}), 3.74(\mathrm{~s}, 3 \mathrm{H})$.

\section{(Z)-N-(4-Chlorobenzylidene)cyclohexanamine oxide (3ae)}

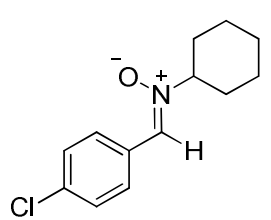

Conditions A: Reaction time: $16 \mathrm{~h}$. Yield: $97 \%$.

Conditions B: Reaction time: 3 min. Yield: $100 \%$

White solid. m.p. $98-99^{\circ} \mathrm{C} .{ }^{1} \mathrm{H}$ NMR $\left(300 \mathrm{MHz}^{\mathrm{CDCl}} 3\right) \delta 8.21$ (d, J = $9.2 \mathrm{~Hz}$, $2 \mathrm{H}), 7.48-7.31(\mathrm{~m}, 3 \mathrm{H}), 3.98-3.72(\mathrm{~m}, 1 \mathrm{H}), 2.20-1.84(\mathrm{~m}, 6 \mathrm{H}), 1.71(\mathrm{~d}, J=11.5$

$\mathrm{Hz}, 1 \mathrm{H}), 1.50-1.14(\mathrm{~m}, 3 \mathrm{H})$.

${ }^{13} \mathrm{C}$ NMR $\left(75 \mathrm{MHz}, \mathrm{CDCl}_{3}\right) \delta 135.4(\mathrm{CH}=\mathrm{N}), 131.2(\mathrm{C}), 129.8(2 \times \mathrm{CH}), 129.4(\mathrm{C}), 128.8(2 \times \mathrm{CH}), 75.9$ $(\mathrm{CH}), 31.2(2 \mathrm{CH}), 25.1(3 \mathrm{CH})$.

MS (ESI+): m/z $238\left(\mathrm{M}^{+}+1\right)(100), 260\left(\mathrm{M}^{+}+\mathrm{Na}\right)$ (20). HRMS (ESI+): calculated for $\mathrm{C}_{13} \mathrm{H}_{16} \mathrm{NONaCl}$ $\left(\mathrm{M}^{+}+\mathrm{Na}\right): 260.0812$; found: 260.0811 .

\section{(Z)-N-(2-Phenylpropylidene)cyclohexanamine oxide (3ue $)^{14}$}

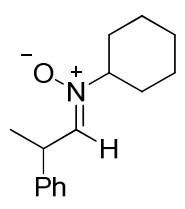

Reaction performed only under conditions B: Reaction time: $3 \mathrm{~min}$. The crude of the reaction was filtered through basic $\mathrm{Al}_{2} \mathrm{O}_{3}$ instead of silica gel. Yield: $94 \%$.

${ }^{1} \mathbf{H}$ NMR $\left(300 \mathrm{MHz}, \mathrm{CDCl}_{3}\right) \delta 7.44-7.16(\mathrm{~m}, 5 \mathrm{H}), 6.84(\mathrm{~d}, J=7.3 \mathrm{~Hz}, 1 \mathrm{H}), 4.55-$ $4.32(\mathrm{~m}, 1 \mathrm{H}), 3.78-3.54(\mathrm{~m}, 1 \mathrm{H}), 2.12-1.77(\mathrm{~m}, 6 \mathrm{H}), 1.75-1.59(\mathrm{~m}, 1 \mathrm{H}), 1.49(\mathrm{~d}$, $J=7.1 \mathrm{~Hz}, 3 \mathrm{H}), 1.42-1.10(\mathrm{~m}, 3 \mathrm{H})$. 


\section{(Z)-1-(4-Chlorophenyl)- $N$-phenylmethanimine oxide (3aa) ${ }^{28}$}

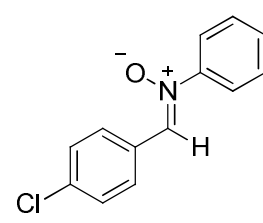

$\mathrm{N}$-phenylhydroxylamine $\mathbf{2 a}$ is commercially available and therefore the reaction was carried out with under catalytic conditions.

The title compound has been successfully synthetized under different conditions:

- Reaction was performed by mixing an equimolecular amount of reagents $(0.2 \mathrm{mmol})$ and $(L)$-proline (10 mol\%) as catalyst instead of pyrrolidine in $\mathrm{MeOH}$ as solvent. After stirring the reaction mixture for $48 \mathrm{~h}$, nitrone 3 aa was purified by flash chromatography (Cyclohexane/EtOAc; 3:1) and obtained in a 92\% isolated yield.

- Reaction was performed by mixing an equimolecular amount of reagents $(0.2 \mathrm{mmol})$ and pyrrolidine (20 mol\%) as catalyst in $\mathrm{CH}_{2} \mathrm{Cl}_{2}$ as solvent. After stirring the reaction mixture for $5 \mathrm{~h}$, nitrone 3aa was filtered through silica gel with EtOAc and obtained in a $94 \%$ isolated yield.

- In order to mimic the optimized conditions B (as if the reaction was performed with the corresponding hydrochloride), reaction was carried out by mixing an equimolecular amount of reagents $(0.2 \mathrm{mmol})$ and a combination of pyrrolidinum chloride $(21.5 \mathrm{mg}$, $0.2 \mathrm{mmol}, 1$ equiv) and pyrrolidine (20 mol\%) as catalyst in dichloromethane. After stirring the reaction during $15 \mathrm{~min}$, the crude of the reaction was filtered through silica gel yielding nitrone $3 a a$ in $96 \%$.

${ }^{1} \mathrm{H}$ NMR $\left(300 \mathrm{MHz}, \mathrm{CDCl}_{3}\right) \delta 8.36(\mathrm{~d}, J=8.8 \mathrm{~Hz}, 2 \mathrm{H}), 7.90(\mathrm{~s}, 1 \mathrm{H}), 7.80-7.71(\mathrm{~m}, 2 \mathrm{H}), 7.51-$ $7.39(\mathrm{~m}, 5 \mathrm{H})$.

Pyrrolidinum chloride was prepared by mixing pyrrolidine $(455,3 \mu \mathrm{L}, 5.45 \mathrm{mmol})$ with $6 \mathrm{~mL}$ of a solution of $\mathrm{HCl} / \mathrm{Et}_{2} \mathrm{O}(1 \mathrm{M}, 1.1$ equiv) and stirring the mixture at room temperature during 2 h. Then, solvent was removed under vacuum and the final product was used without further purification.

\section{Synthesis of cerovive (NXY-059) $)^{29}$}
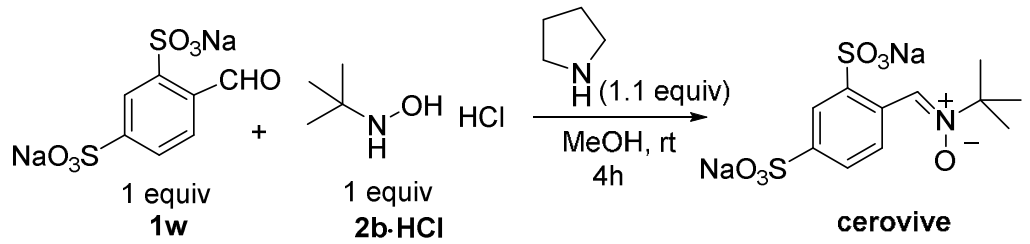

To a solution (0.4 M) of an equimolar amount of $\mathrm{N}$-hydroxylamine hydrochloride $\mathbf{2 b} \cdot \mathbf{H C l}(0.8$ $\mathrm{mmol}$ ) and aldehyde $\mathbf{1} \mathbf{w}$ in $\mathrm{MeOH}, 1.1$ equiv of pyrrolidine $(73.1 \mu \mathrm{L})$ was added. The mixture was stirred at room temperature for $4 \mathrm{~h}$. Then, 1 equiv of $\mathrm{Na}_{2} \mathrm{CO}_{3} \cdot \mathrm{H}_{2} \mathrm{O}(99 \mathrm{mg})$ was added to the mixture and stirred for $1 \mathrm{~h}$, whereupon it was filtered through a small cotton to remove the solid 
$\mathrm{NaHCO}_{3}$. Cool $\mathrm{Et}_{2} \mathrm{O}$ was added to the filtrate and the resulting precipitate was filtered and washed with $2 \times 5 \mathrm{~mL}$ of a cool mixture of $\mathrm{MeOH} / \mathrm{Et}_{2} \mathrm{O}(1: 2)$ to provide cerovive in $90 \%$ yield.

\section{Effect of water and the amount of pyrrolidine in the formation of $\mathbf{3} \mathbf{c b}, \mathbf{3} \mathbf{d b}$ and $\mathbf{3 p b}$}

We observed that the use of water as co-solvent in the formation of nitrones $\mathbf{3} \mathbf{c b}, \mathbf{3} \mathbf{d b}$ and $3 \mathrm{pb}$, was detrimental to reach complete conversion.

The reaction of $p$-anisaldehyde $\mathbf{1 c}$ or $p$-nitrobenzaldehyde $\mathbf{1 d}$ with $N$-tert butylhydroxylamine hydrochloride $\mathbf{2} \mathbf{b} \cdot \mathbf{H C l}$ using 1 equiv of pyrrolidine in $\mathrm{MeOH}$ provided complete conversions after $21 \mathrm{~h}$ (entries 1 and 5). Nevertheless, when water was used as co-solvent, lower conversions were obtained for both aldehydes $\mathbf{1 c}$ (entry 2) and 1d (entry 6). The use of 1.1 equiv of pyrrolidine accelerates the reaction. This effect is larger when $\mathrm{MeOH}$ was used as solvent (compare reaction times of entries 1 and 5 and 4 y 8 ).

Remarkably, the reaction with 3-indolylcarboxaldehyde $3 p$ did not provided the final product in $\mathrm{H}_{2} \mathrm{O} / \mathrm{MeOH}$, not even with excess of pyrrolidine (entries 9, 10). However, the final nitrone $\mathbf{3 p b}$ was obtained in quantitative yield using $\mathrm{MeOH}$ as solvent and 1.1 equiv of pyrrolidine (entry 11).

Table S5. Effect of water and the amount of pyrrolidine

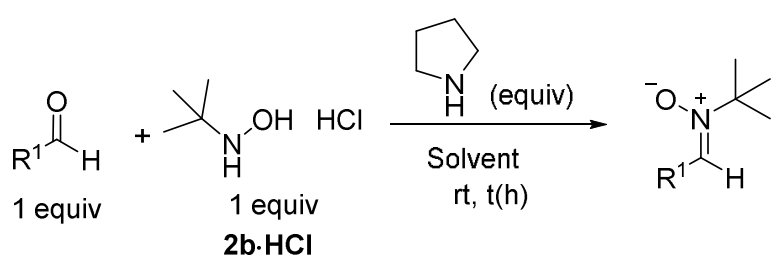

\begin{tabular}{|c|c|c|c|c|c|c|}
\hline Entry & $\begin{array}{c}\text { Pyrrolidine } \\
\text { (equiv) }\end{array}$ & $1, R^{1}$ & Solvent & $t(h)$ & Conv. (\%) & $\begin{array}{l}\text { Yield } \\
(\%)\end{array}$ \\
\hline 1 & 1 & 1c, $p-\mathrm{MeOC}_{6} \mathrm{H}_{4}$ & $\mathrm{MeOH}$ & 21 & $3 c b 100$ & 100 \\
\hline 2 & 1 & 1c, $p-\mathrm{MeOC}_{6} \mathrm{H}_{4}$ & $\mathrm{H}_{2} \mathrm{O} / \mathrm{MeOH}$ & 26 & $3 c b 66$ & nd \\
\hline 3 & 1.1 & 1c, $p-\mathrm{MeOC}_{6} \mathrm{H}_{4}$ & $\mathrm{H}_{2} \mathrm{O} / \mathrm{MeOH}$ & 23 & $3 c b 75$ & nd \\
\hline 4 & 1.1 & 1c, $p-\mathrm{MeOC}_{6} \mathrm{H}_{4}$ & $\mathrm{MeOH}$ & 8 & $3 c b 100$ & 90 \\
\hline 5 & 1 & 1d, $p-\mathrm{NO}_{2} \mathrm{C}_{6} \mathrm{H}_{4}$ & $\mathrm{MeOH}$ & 21 & $3 \mathbf{d b} 100$ & 95 \\
\hline 6 & 1 & 1d, $p-\mathrm{NO}_{2} \mathrm{C}_{6} \mathrm{H}_{4}$ & $\mathrm{H}_{2} \mathrm{O} / \mathrm{MeOH}$ & 26 & $3 d b 87$ & nd \\
\hline 7 & 1.1 & 1d, $p-\mathrm{NO}_{2} \mathrm{C}_{6} \mathrm{H}_{4}$ & $\mathrm{H}_{2} \mathrm{O} / \mathrm{MeOH}$ & 23 & $3 d b 82$ & nd \\
\hline 8 & 1.1 & $1 \mathrm{~d}, p-\mathrm{NO}_{2} \mathrm{C}_{6} \mathrm{H}_{4}$ & $\mathrm{MeOH}$ & 8.5 & $3 \mathrm{db} 100$ & 90 \\
\hline 9 & 1 & 1p, 3-indolyl & $\mathrm{H}_{2} \mathrm{O} / \mathrm{MeOH}$ & 16 & $3 p b 0$ & - \\
\hline 10 & 1.1 & 1p, 3-indolyl & $\mathrm{H}_{2} \mathrm{O} / \mathrm{MeOH}$ & 21 & $3 p b 0$ & - \\
\hline 11 & 1.1 & 1p, 3-indolyl & $\mathrm{MeOH}$ & 29 & $3 p b 100$ & 100 \\
\hline
\end{tabular}

nd: not determined. 


\section{REFERENCES}

1. Waterbury, L. D.; Carney, J. M.; Wilcox, A. L. PCT Int. Appl. 2000003977, 2000

2. Prakash, G. K. S.; Zhang, Z.; Wang, F.; Rahm, M.; Ni, C.; Iuliucci, M.; Haiges, R.; Olah, G. A. Chem. Eur. J. 2014, 20, 831-838.

3. Gautheron-Chapoulaud, V.; Pandya, S. U.; Cividino, P.; Masson, G.; Py, S.; Vallée, Y. Synlett 2001, 12811283.

4. Kim, S.; Vilela, G. V. M.; Bouajila, J.; Dias, A. G.; Cyrino, F.; Bouskela, E.; Costa, P. R.; Nepveu, F. Bioorg. Med. Chem. 2007, 15, 3572-3578.

5. Canterbury, D. P.; Herrick, I. R.; Um, J.; Houk, K. N.; Frontier, A. J. Tetrahedron 2009, 65, 3165-3179.

6. Khan, M. A.; Upasani, R. B.; Wood, P. L. PCT Int. Appl. 2004034999, 2004.

7. Colacino, E.; Nun, P.; Colacino, M. F.; Martinez, J.; Lamaty, F. Tetrahedron 2008, 64, 5569-5576

8. Andrade, M. M.; Barros, M. T.; Pinto, R. C. Tetrahedron 2008, 64, 10521-10530.

9. Valizadeh, H. Heteroat. Chem. 2010, 21, 78-83.

10. Samadi, A.; Soriano, E.; Revuelta, J.; Valderas, C.; Chioua, M.; Garrido, I.; Bartolomé, B.; Tomassolli, I.; Ismaili, L.; González-Lafuente, L.; Villarroya, M.; García, A G.; Oset-Gasque, M. J.; Marco-Contelles, J. Bioorg. Med. Chem. 2011, 19, 951-960.

11. Evans, D. A.; Song, H.-J.; Fandrick, K. R. Org. Lett. 2006, 8, 3351-3354.

12. Aschwanden, P.; Kvrno, L.; Geisser, R. W.; Kleinbeck, F.; Carreira, E. M. Org. Lett. 2005, 7, 5741-5742.

13. Dias, A. G.; Santos, C. E. V.; Cyrino, F. Z. G. A.; Bouskela, E.; Costa, P. R. R. Bioorg. Med. Chem. 2009, 17, 3995- 3998.

14. Coates, R. M.; Cummins, C. H. J. Org. Chem. 1986, 51, 1383-1389.

15. Liard, A.; Nguyen, T.-H.; Djelloul Smir, A. I.; Vaultier, M.; Derdour, A.; Mortier, J. Chem. Eur. J. 2003, 9, 1000-1007.

16. Blixt, J. PCT Int. Appl. 2000002848, 2000.

17. U. Jahn and W. Schroth, Tetrahedron Lett. 1993, 34, 5863-5866.

18. $p$-Chlorobenzaldehyde was washed with $\mathrm{NaHCO}_{3}$ before using it to remove acids traces.

19. Under these conditions, reactions were monitored by ${ }^{1} \mathrm{H}$ NMR until complete conversion.

20. It is important to wash several times with EtOAc for recovering all the mass.

21. Murray, R. W.; Singh, M. J. Org. Chem. 1990, 55, 2954-2957.

22. Xing, D.; Xu, X.; Yang, L. Synthesis 2009, 3399-3404.

23. Chavarria, C.; Pérez, D. I.; Pérez, C.; Morales García, J. A.; Alonso-Gil, S.; Pérez-Castillo, A.; Gil, C.; Souza, J. M.; Porcal, W. Eur. J. Med. Chem. 2012, 58, 44-49.

24. Cummins, C. H.; Coates, R. M. J. Org. Chem. 1983, 48, 2070-2076.

25. Kano, T.; Hashimoto, T.; Maruoka, K. J. Am. Chem. Soc. 2005, 127, 11926-11927.

26. Tyrrell, E.; Allen, J.; Jones, K.; Beauchet, R. Synthesis 2005, 2393-2329.

27. Zheng, H.; McDonald, R.; Hall, D. G. Chem. Eur. J. 2010, 16, 5454-5460.

28. Murray, R. W.; Singh, M. J. Org. Chem. 1990, 55, 2954-2957.

29. Blixt, J.; Kruk, H.; Mcginley, J.; Pouhov, S.; Vajdaal, J. PCT Int. Appl. 2001051460, 2001. 


\section{THEORETICAL CALCULATIONS}

All calculations were performed with Gaussian 09 at DFT level. ${ }^{30}$ The geometries of all the intermediates and transition states were fully optimized in methanol or dichloromethane, with $\mathrm{CPCM}^{31}$ model, at the DFT ( $\left.\mathrm{wB97xd}\right)^{32}$ level using TZVP ${ }^{33}$ basis set for all the atoms. The frecuencies and thermoquimic corrections at $298 \mathrm{~K}$ and $1 \mathrm{~atm}$ were also computed at the same level of theory.

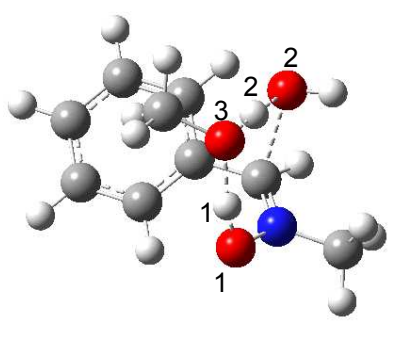

TSIMeOH (24.5)
$\mathrm{C}-\mathrm{N}: 1.30$

C-O2: 2.23

$\mathrm{O}^{1}-\mathrm{H}^{1}: 1.05$

$\mathrm{O}^{3}-\mathrm{H}^{1}: 1.47$

$\mathrm{O}^{3}-\mathrm{H}^{2}: 1.11$

$\mathrm{O}^{2}-\mathrm{H}^{2}: 1.33$

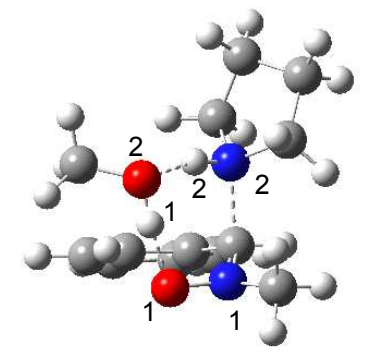

TSIIIMeOH (19.5)
C-N $\mathrm{N}^{1}: 1.35$

C-N²: 1.85

$\mathrm{O}^{1}-\mathrm{H}^{1}: 1.54$

$\mathrm{O}^{2}-\mathrm{H}^{1}: 1.02$

$\mathrm{O}^{2}-\mathrm{H}^{2}: 1.84$

$\mathrm{N}^{2}-\mathrm{H}^{2}: 1.03$

Figure S1. Optimized geometries for transition states TSI and TSIII including one molecule of $\mathrm{MeOH}$ acting as proton relay. Relevant distances are indicated in $\AA$. Free activation barriers $\left(\mathrm{kcal} \cdot \mathrm{mol}^{-1}\right)$ from hemiaminal I and aminal III are also indicated in parentheses.

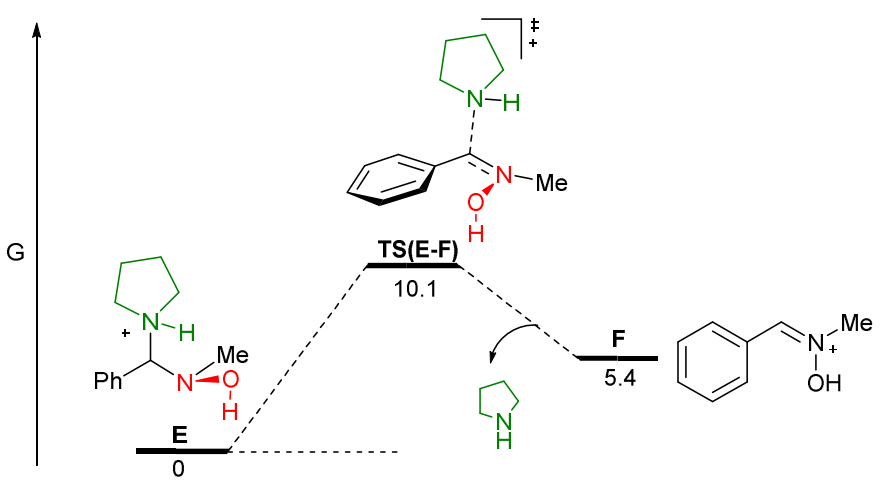

Figure S2. Calculated free energy profile $\left(\mathrm{CPCM}_{\mathrm{MeOH}} \mathrm{WB97xd} / \mathrm{tzvp}\right.$, energies in $\mathrm{kcal} \mathrm{mol}^{-1}$ at 298 K) for the transformation of intermediate $\mathbf{E}$ in $\mathbf{F}$.

30 Gaussian 09, Revision D.01, Frisch, M. J.; Trucks, G. W.; Schlegel, H. B.; Scuseria, G. E.; Robb, M. A.; Cheeseman, J. R.; Scalmani, G.; Barone, V.; Mennucci, B.; Petersson, G. A.; Nakatsuji, H.; Caricato, M.; Li, X.; Hratchian, H. P.; Izmaylov, A. F.; Bloino, J.; Zheng, G.; Sonnenberg, J. L.; Hada, M.; Ehara, M.; Toyota, K.; Fukuda, R.; Hasegawa, J.; Ishida, M.; Nakajima, T.; Honda, Y.; Kitao, O.; Nakai, H.; Vreven, T.; Montgomery, J. A. Jr., Peralta, J. E.; Ogliaro, F.; Bearpark, M.; Heyd, J. J.; Brothers, E.; Kudin, K. N.; Staroverov, V. N.; Keith, T.; Kobayashi, R.; Normand, J.; Raghavachari, K.; Rendell, A.; Burant, J.C.; lyengar, S. S.; Tomasi, J.; Cossi, M.; Rega, N.; Millam, J. M.; Klene, M.; Knox, J. E.; Cross, J. B.; Bakken, V.; Adamo, C.; Jaramillo, J.; Gomperts, R.; Stratmann, R. E.; Yazyev, O.; Austin, A. J.; Cammi, R.; Pomelli, C.; Ochterski, J. W.; Martin, R, L.; Morokuma, K.; Zakrzewski, V. G.; Voth, G. A.; Salvador, P.; Dannenberg, J. J.; Dapprich, S.; Daniels, A. D.; Farkas, O.; Foresman, J. B.; Ortiz, J. V.; Cioslowski, J.; Fox, D. J. Gaussian, Inc., Wallingford CT, 2013.

31 (a) Barone, V.; Cossi, M. J. Phys. Chem. A 1998, 102, 1995-2001. (b) Cossi, M.; Rega, N.; Scalmani G.; Barone, V. J. Comput. Chem. 2003, 24, 669-681.

32 Chai, J.-D.; Head-Gordon, M. Phys. Chem. Phys., 2008, 10, 6615-6620.

33 Schaefer,A.; Huber, C.; Ahlrichs, R. J. Chem. Phys., 1994, 100, 5829-5835. 


\section{Cartesian coordinates $(\AA)$ and energies (hartrees) of all the optimized structures.}

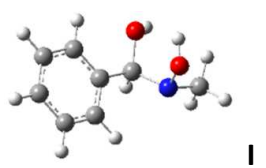

$E($ wB97xd / tzvp $)=-516.628183306$

$H($ correction $)=0.195601$

$\mathrm{G}($ correction $)=0.148060$

Imaginary frequencies: 0

\begin{tabular}{lrrrr}
6 & 0 & -0.989281 & -0.299216 & -0.626951 \\
1 & 0 & -1.163274 & -0.075013 & -1.683273 \\
6 & 0 & 0.482799 & -0.115021 & -0.317680 \\
7 & 0 & -1.791383 & 0.661418 & 0.112890 \\
8 & 0 & -1.361506 & -1.639511 & -0.347702 \\
6 & 0 & 1.046088 & 1.152198 & -0.445674 \\
6 & 0 & 1.290269 & -1.181175 & 0.054528 \\
8 & 0 & -1.695332 & 0.372079 & 1.495501 \\
6 & 0 & -3.195078 & 0.663853 & -0.270434 \\
1 & 0 & -2.092518 & -1.889866 & -0.915667 \\
6 & 0 & 2.395509 & 1.350325 & -0.200076 \\
1 & 0 & 0.417471 & 1.986289 & -0.732815 \\
6 & 0 & 2.643090 & -0.982027 & 0.306110 \\
1 & 0 & 0.858762 & -2.167897 & 0.148893 \\
1 & 0 & -1.934802 & -0.563584 & 1.586196 \\
1 & 0 & -3.695627 & -0.293991 & -0.073335 \\
1 & 0 & -3.708108 & 1.444704 & 0.287459 \\
1 & 0 & -3.265569 & 0.890979 & -1.334752 \\
6 & 0 & 3.199749 & 0.281518 & 0.179446 \\
1 & 0 & 2.822688 & 2.340212 & -0.304782 \\
1 & 0 & 3.262212 & -1.820619 & 0.601151 \\
1 & 0 & 4.254263 & 0.435587 & 0.372686 \\
\hline & & & & \\
\hline & & &
\end{tabular}

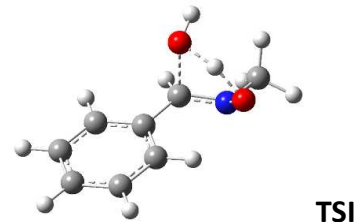

$E(w B 97 x d / \operatorname{tzvp})=-516.574911233$

$\mathrm{H}($ correction $)=0.190355$

$\mathrm{G}($ correction $)=0.143171$

Imaginary frequencies: $1\left(-532.6700 \mathrm{~cm}^{-1}\right)$

$\begin{array}{lllll}6 & 0 & -0.911401 & -0.592450 & -0.385751\end{array}$

$\begin{array}{llrrr}1 & 0 & -1.093641 & -1.584653 & -0.784944 \\ 6 & 0 & 0.497277 & -0.228350 & -0.229067 \\ 7 & 0 & -1.921491 & 0.239425 & -0.523752 \\ 8 & 0 & -1.195251 & -0.664976 & 1.765648 \\ 6 & 0 & 0.953996 & 1.087120 & -0.124068 \\ 6 & 0 & 1.422380 & -1.273210 & -0.256177 \\ 8 & 0 & -1.938291 & 1.239955 & 0.419635 \\ 6 & 0 & -3.247105 & -0.227996 & -0.888624 \\ 1 & 0 & -1.944326 & -1.241134 & 1.939267 \\ 6 & 0 & 2.312250 & 1.341199 & -0.035304 \\ 1 & 0 & 0.244163 & 1.901320 & -0.106439 \\ 6 & 0 & 2.779156 & -1.014601 & -0.155220 \\ 1 & 0 & 1.074407 & -2.294945 & -0.352740 \\ 1 & 0 & -1.671341 & 0.597287 & 1.240280 \\ 1 & 0 & -3.755023 & -0.630902 & -0.009216 \\ 1 & 0 & -3.809001 & 0.616767 & -1.279792 \\ 1 & 0 & -3.160486 & -0.989696 & -1.660240 \\ 6 & 0 & 3.226605 & 0.294844 & -0.043994 \\ 1 & 0 & 2.660625 & 2.363296 & 0.043761 \\ 1 & 0 & 3.487510 & -1.832980 & -0.170185 \\ 1 & 0 & 4.286937 & 0.500502 & 0.033493---\end{array}$

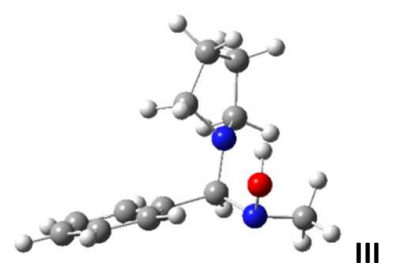

$E($ wB97xd / tzvp $)=-652.788090119$

$\mathrm{H}$ (correction) $=0.305944$

$\mathrm{G}$ (correction) $=0.250078$

Imaginary frequencies: 0

$\begin{array}{lllll}7 & 0 & -1.265404 & -0.216262 & -0.044419 \\ 6 & 0 & -2.100401 & -0.910430 & -1.022875 \\ 6 & 0 & -0.887906 & -1.244115 & 0.925463 \\ 6 & 0 & -0.251422 & 0.688763 & -0.583018 \\ 6 & 0 & -2.977521 & -1.832643 & -0.167493 \\ 1 & 0 & -1.480201 & -1.503928 & -1.712170 \\ 1 & 0 & -2.674553 & -0.198076 & -1.616010 \\ 1 & 0 & -0.138809 & -1.934267 & 0.511156 \\ 1 & 0 & -0.467166 & -0.799232 & 1.826895 \\ 6 & 0 & -2.208244 & -1.974406 & 1.167799 \\ 1 & 0 & -0.377273 & 0.728639 & -1.666700\end{array}$ 


$\begin{array}{lllll}6 & 0 & 1.171256 & 0.236903 & -0.323654 \\ 7 & 0 & -0.494839 & 2.071514 & -0.127567 \\ 1 & 0 & -3.958044 & -1.386914 & -0.002069 \\ 1 & 0 & -3.131044 & -2.792637 & -0.659241 \\ 1 & 0 & -2.043866 & -3.013911 & 1.449299 \\ 1 & 0 & -2.758563 & -1.493387 & 1.976971 \\ 6 & 0 & 1.734152 & -0.690361 & -1.196946 \\ 6 & 0 & 1.919898 & 0.675541 & 0.764094 \\ 6 & 0 & 3.014970 & -1.181074 & -0.985706 \\ 1 & 0 & 1.164342 & -1.033982 & -2.053586 \\ 6 & 0 & 3.202311 & 0.187711 & 0.976018 \\ 1 & 0 & 1.491381 & 1.395769 & 1.447899 \\ 6 & 0 & 3.753166 & -0.743702 & 0.105287 \\ 1 & 0 & 3.436742 & -1.900994 & -1.676411 \\ 1 & 0 & 3.773875 & 0.537198 & 1.827469 \\ 1 & 0 & 4.753673 & -1.122866 & 0.273689 \\ 1 & 0 & -1.498361 & 1.333687 & 1.304076 \\ 6 & 0 & -1.618748 & 2.680317 & -0.827922 \\ 1 & 0 & -2.550475 & 2.110791 & -0.705910 \\ 1 & 0 & -1.767785 & 3.684847 & -0.435604 \\ 1 & 0 & -1.377804 & 2.751919 & -1.889123 \\ 8 & 0 & -0.822930 & 2.036944 & 1.252123 \\ -------------------------------------------------------\end{array}$

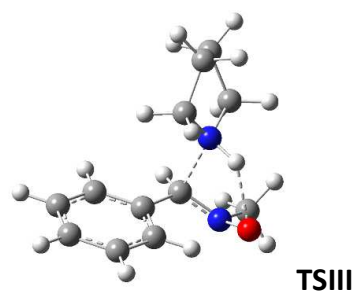

$E(w B 97 x d /$ tzvp $)=-652.750598771$

$\mathrm{H}$ (correction) $=0.304846$

$\mathrm{G}($ correction $)=0.249309$

Imaginary frequencies: $1\left(-369.7144 \mathrm{~cm}^{-1}\right)$

$\begin{array}{lllll}7 & 0 & 1.297480 & -0.271552 & -0.319715 \\ 6 & 0 & 2.649402 & -0.348649 & 0.290270 \\ 6 & 0 & 0.843080 & -1.641135 & -0.647393 \\ 6 & 0 & 0.151106 & 0.897278 & 0.588819 \\ 6 & 0 & 2.929748 & -1.840649 & 0.466487 \\ 1 & 0 & 2.668724 & 0.194663 & 1.234605 \\ 1 & 0 & 3.370978 & 0.119022 & -0.382614 \\ 1 & 0 & 0.159670 & -1.980449 & 0.133894 \\ 1 & 0 & 0.301289 & -1.637230 & -1.590937 \\ 6 & 0 & 2.114001 & -2.481985 & -0.654485 \\ 1 & 0 & 0.471690 & 0.749981 & 1.616198 \\ 6 & 0 & -1.192050 & 0.315293 & 0.319751 \\ 7 & 0 & 0.494366 & 2.101833 & 0.098696 \\ 1 & 0 & 3.994308 & -2.061068 & 0.408999\end{array}$

$\begin{array}{llrrr}1 & 0 & 2.563683 & -2.181537 & 1.437257 \\ 1 & 0 & 1.908971 & -3.538388 & -0.487071 \\ 1 & 0 & 2.631102 & -2.377973 & -1.610891 \\ 6 & 0 & -1.804686 & -0.395955 & 1.351182 \\ 6 & 0 & -1.851274 & 0.438757 & -0.903695 \\ 6 & 0 & 1.620119 & 2.810370 & 0.673562 \\ 8 & 0 & 0.413059 & 2.246562 & -1.260213 \\ 6 & 0 & -3.056030 & -0.967782 & 1.172228 \\ 1 & 0 & -1.299149 & -0.502648 & 2.304453 \\ 6 & 0 & -3.103885 & -0.130686 & -1.078133 \\ 1 & 0 & -1.361269 & 0.990900 & -1.693305 \\ 1 & 0 & 2.560628 & 2.500590 & 0.200955 \\ 1 & 0 & 1.483750 & 3.876174 & 0.489917 \\ 1 & 0 & 1.675282 & 2.640260 & 1.749802 \\ 1 & 0 & 1.294082 & 0.333484 & -1.146312 \\ 6 & 0 & -3.709094 & -0.835722 & -0.045235 \\ 1 & 0 & -3.519161 & -1.515030 & 1.983886 \\ 1 & 0 & -3.609479 & -0.027342 & -2.030620 \\ 1 & 0 & -4.685111 & -1.282683 & -0.189520\end{array}$

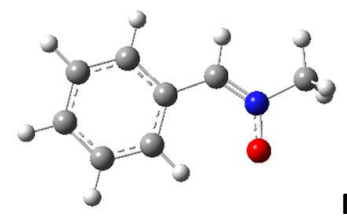

Model nitrone

$E(w B 97 x d /$ tzvp $)=-440.180273532$

$\mathrm{H}($ correction $)=0.166161$

$\mathrm{G}($ correction $)=0.122065$

Imaginary frequencies: 0

$\begin{array}{llrrr}1 & 0 & -0.030356 & 1.821837 & 0.000032 \\ 6 & 0 & -0.758017 & 1.025827 & 0.000011 \\ 6 & 0 & -2.621433 & -1.044040 & -0.000046 \\ 6 & 0 & -0.313554 & -0.302839 & -0.000010 \\ 6 & 0 & -2.118030 & 1.303932 & 0.000003 \\ 6 & 0 & -3.051864 & 0.278601 & -0.000026 \\ 6 & 0 & -1.269114 & -1.330744 & -0.000038 \\ 1 & 0 & -2.448512 & 2.335481 & 0.000019 \\ 1 & 0 & -4.110981 & 0.504805 & -0.000032 \\ 1 & 0 & -0.940340 & -2.363805 & -0.000054 \\ 1 & 0 & -3.343243 & -1.851151 & -0.000068 \\ 6 & 0 & 1.079286 & -0.713919 & -0.000003 \\ 1 & 0 & 1.291655 & -1.772928 & -0.000015 \\ 7 & 0 & 2.123128 & 0.060804 & 0.000017 \\ 6 & 0 & 3.471652 & -0.528527 & 0.000035 \\ 1 & 0 & 3.984837 & -0.169044 & 0.889202 \\ 1 & 0 & 3.984908 & -0.168919 & -0.889039 \\ 1 & 0 & 3.421194 & -1.613631 & -0.000043 \\ 8 & 0 & 2.101923 & 1.340248 & 0.000039\end{array}$




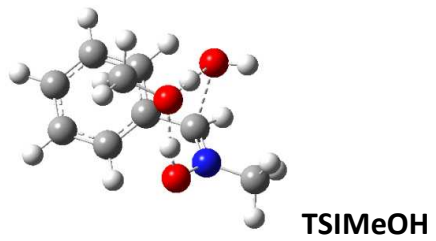

$E($ wB97xd / tzvp $)=-632.339374506$

$\mathrm{H}$ (correction) $=0.246844$

$\mathrm{G}($ correction $)=0.191171$

Imaginary frequencies: $1\left(-437.5409 \mathrm{~cm}^{-1}\right)$

$\begin{array}{llrrc}6 & 0 & -0.772624 & -0.988497 & 0.602206 \\ 1 & 0 & -0.976386 & -1.512378 & 1.523432 \\ 6 & 0 & 0.630603 & -0.697055 & 0.274352 \\ 7 & 0 & -1.748028 & -1.001780 & -0.253434 \\ 8 & 0 & -1.117186 & 0.868866 & 1.792281 \\ 6 & 0 & 1.144056 & -0.889627 & -1.008184 \\ 6 & 0 & 1.486699 & -0.316923 & 1.307872 \\ 8 & 0 & -1.675737 & -0.298064 & -1.426903 \\ 6 & 0 & -3.095810 & -1.424998 & 0.077488 \\ 1 & 0 & -1.986677 & 0.782356 & 2.190284 \\ 1 & 0 & -1.226802 & 1.530148 & 0.640298 \\ 6 & 0 & 2.492542 & -0.680011 & -1.252510 \\ 1 & 0 & 0.491568 & -1.197196 & -1.812194 \\ 6 & 0 & 2.829732 & -0.098455 & 1.055163 \\ 1 & 0 & 1.073127 & -0.157368 & 2.293731 \\ 1 & 0 & -3.704690 & -0.543918 & 0.285154 \\ 1 & 0 & -3.506822 & -1.961940 & -0.774290 \\ 1 & 0 & -3.069342 & -2.077109 & 0.946707 \\ 6 & 0 & 3.335808 & -0.277177 & -0.226541 \\ 1 & 0 & 2.884801 & -0.831370 & -2.250147 \\ 1 & 0 & 3.485156 & 0.211068 & 1.859337 \\ 1 & 0 & 4.386800 & -0.106035 & -0.423542 \\ 1 & 0 & -1.502709 & 0.684168 & -1.114729 \\ 8 & 0 & -1.280552 & 1.942689 & -0.387021 \\ 6 & 0 & -0.089017 & 2.636882 & -0.682577 \\ 1 & 0 & 0.102566 & 3.413783 & 0.065120 \\ 1 & 0 & -0.178383 & 3.118386 & -1.659011 \\ 1 & 0 & 0.779852 & 1.967091 & -0.706584 \\ ----------------------------------------------------\end{array}$

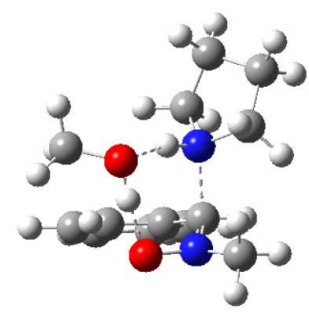

TSIIIMeOH
$E(w B 97 x d /$ tzvp $)=-768.511841322$

$\mathrm{H}$ (correction) $=0.362861$

$\mathrm{G}$ (correction) $=0.297709$

Imaginary frequencies: $1\left(-271.8311 \mathrm{~cm}^{-1}\right)$

$\begin{array}{lllll}6 & 0 & -0.072821 & -0.160339 & -1.207717\end{array}$

$\begin{array}{llllll}1 & 0 & 0.100004 & 0.545245 & -2.015245\end{array}$

$\begin{array}{llllll}6 & 0 & -1.408837 & 0.024511 & -0.568276\end{array}$

$\begin{array}{llllll}7 & 0 & 0.351520 & -1.395072 & -1.532463\end{array}$

$\begin{array}{llllll}7 & 0 & 1.082422 & 0.629081 & 0.005177\end{array}$

$\begin{array}{llllll}6 & 0 & -1.854820 & -0.735461 & 0.511942\end{array}$

$\begin{array}{llllll}6 & 0 & -2.230928 & 1.033657 & -1.067959\end{array}$

$\begin{array}{llllll}8 & 0 & 0.220298 & -2.426347 & -0.634350\end{array}$

$\begin{array}{llllll}6 & 0 & 1.475925 & -1.565852 & -2.426495\end{array}$

$\begin{array}{llllll}6 & 0 & 0.557544 & 1.770699 & 0.784819\end{array}$

$\begin{array}{llllll}6 & 0 & 2.354868 & 1.071100 & -0.630747\end{array}$

$\begin{array}{llllll}6 & 0 & -3.099153 & -0.490042 & 1.072547\end{array}$

$\begin{array}{llllll}1 & 0 & -1.225337 & -1.526006 & 0.889467\end{array}$

$\begin{array}{llllll}6 & 0 & -3.477035 & 1.276407 & -0.507838\end{array}$

$\begin{array}{llllll}1 & 0 & -1.893512 & 1.636853 & -1.903185\end{array}$

$\begin{array}{llllll}1 & 0 & 1.145035 & -2.139664 & 0.569051\end{array}$

$\begin{array}{llllll}1 & 0 & 2.424726 & -1.566420 & -1.876156\end{array}$

$\begin{array}{llllll}1 & 0 & 1.370239 & -2.528411 & -2.926346\end{array}$

$\begin{array}{llllll}1 & 0 & 1.493590 & -0.774167 & -3.176401\end{array}$

$\begin{array}{llllll}6 & 0 & 1.802629 & 2.532865 & 1.212241\end{array}$

$\begin{array}{lllll}1 & 0 & -0.060149 & 1.406960 & 1.602743\end{array}$

$\begin{array}{llllll}1 & 0 & -0.064970 & 2.384561 & 0.129768\end{array}$

$\begin{array}{llllll}6 & 0 & 2.675239 & 2.453838 & -0.041659\end{array}$

$\begin{array}{llllll}1 & 0 & 3.124397 & 0.332854 & -0.406909\end{array}$

$\begin{array}{llllll}1 & 0 & 2.231047 & 1.112517 & -1.712635\end{array}$

$\begin{array}{llllll}6 & 0 & -3.913479 & 0.515068 & 0.567130\end{array}$

$\begin{array}{llllll}1 & 0 & -3.434088 & -1.087520 & 1.911655\end{array}$

$\begin{array}{llllll}1 & 0 & -4.103929 & 2.062559 & -0.909964\end{array}$

$\begin{array}{llllll}8 & 0 & 1.743773 & -1.799831 & 1.322972\end{array}$

$\begin{array}{lllll}1 & 0 & 1.579934 & 3.555824 & 1.512466\end{array}$

$\begin{array}{lllll}1 & 0 & 2.282209 & 2.024649 & 2.051851\end{array}$

$\begin{array}{llllll}1 & 0 & 3.737181 & 2.569001 & 0.167952\end{array}$

$\begin{array}{llllll}1 & 0 & 2.385576 & 3.235382 & -0.745742\end{array}$

$\begin{array}{llllll}1 & 0 & -4.883805 & 0.703650 & 1.009697\end{array}$

$\begin{array}{llllll}6 & 0 & 1.149849 & -2.084460 & 2.569771\end{array}$

$\begin{array}{llllll}1 & 0 & 1.296970 & -0.154248 & 0.638045\end{array}$

$\begin{array}{llllll}1 & 0 & 0.188985 & -1.570580 & 2.699842\end{array}$

$\begin{array}{llllll}1 & 0 & 1.820843 & -1.744968 & 3.360589\end{array}$

$\begin{array}{llllll}1 & 0 & 0.981015 & -3.158663 & 2.704919\end{array}$ 
$3 \mathrm{H}_{2} \mathrm{O}$

$E($ wB97xd / tzvp $)=-76.443217585$

$\mathrm{H}$ (correction) $=0.025316$

$\mathrm{G}($ correction $)=0.003901$

Imaginary frequencies: 0

$\begin{array}{lllll}8 & 0 & 0.000000 & 0.000000 & 0.117327\end{array}$

$\begin{array}{llllll}1 & 0 & 0.000000 & 0.758651 & -0.469309\end{array}$

$\begin{array}{llllll}1 & 0 & 0.000000 & -0.758651 & -0.469309--\end{array}$

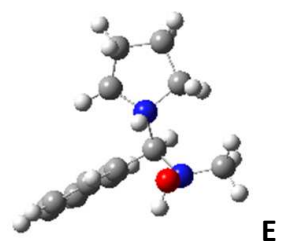

$E($ wB97xd / tzvp $)=-653.246465618$

$\mathrm{H}($ correction $)=0.321262$

$G($ correction $)=0.264113$

Imaginary frequencies: 0

$\begin{array}{rrrrr}7 & 0 & 1.233479 & -0.283610 & -0.235452 \\ 6 & 0 & 2.670902 & -0.112896 & 0.230860 \\ 6 & 0 & 0.934165 & -1.759540 & -0.156035 \\ 1 & 0 & 1.190857 & -0.001284 & -1.216697 \\ 6 & 0 & 0.220921 & 0.622675 & 0.468543 \\ 6 & 0 & 3.244049 & -1.526058 & 0.359021 \\ 1 & 0 & 2.662181 & 0.418093 & 1.177503 \\ 1 & 0 & 3.187476 & 0.490120 & -0.510739 \\ 1 & 0 & 0.586804 & -1.964185 & 0.856110 \\ 1 & 0 & 0.149189 & -2.006465 & -0.862943 \\ 6 & 0 & 2.276209 & -2.404385 & -0.431173 \\ 1 & 0 & 0.451162 & 0.556928 & 1.530661 \\ 6 & 0 & -1.189011 & 0.120864 & 0.240999 \\ 7 & 0 & 0.369809 & 1.997467 & 0.075221 \\ 1 & 0 & 4.262189 & -1.572897 & -0.020132 \\ 1 & 0 & 3.256065 & -1.827218 & 1.406334 \\ 1 & 0 & 2.287226 & -3.443577 & -0.110041 \\ 1 & 0 & 2.497421 & -2.373371 & -1.499409 \\ 6 & 0 & -1.967231 & -0.163734 & 1.357172 \\ 6 & 0 & -1.734653 & -0.043591 & -1.030557 \\ 6 & 0 & 1.441259 & 2.789539 & 0.659713 \\ 8 & 0 & 0.471641 & 2.060069 & -1.336431 \\ 6 & 0 & -3.276876 & -0.598514 & 1.210039 \\ 1 & 0 & -1.549045 & -0.045582 & 2.349933 \\ 6 & 0 & -3.042189 & -0.480175 & -1.176355 \\ 1 & 0 & -1.145217 & 0.156366 & -1.916515 \\ 1 & 0 & 2.427455 & 2.557789 & 0.248835\end{array}$

$\begin{array}{lllll}1 & 0 & 1.225841 & 3.839943 & 0.471750 \\ 1 & 0 & 1.442957 & 2.627207 & 1.737030 \\ 1 & 0 & -0.327437 & 2.512356 & -1.623627 \\ 6 & 0 & -3.816941 & -0.756940 & -0.057139 \\ 1 & 0 & -3.871313 & -0.817368 & 2.087983 \\ 1 & 0 & -3.456469 & -0.608034 & -2.168292 \\ 1 & 0 & -4.837108 & -1.099841 & -0.175209\end{array}$

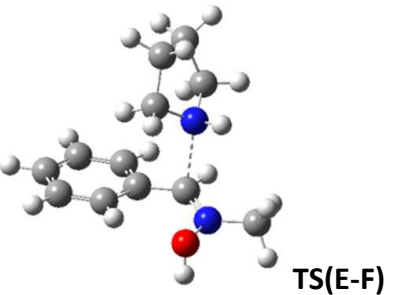

$E($ wB97xd / tzvp $)=-653.224759964$

$\mathrm{H}$ (correction) $=0.318105$

$\mathrm{G}$ (correction) $=0.258439$

Imaginary frequencies: $1\left(-48.9328 \mathrm{~cm}^{-1}\right)$

$\begin{array}{llllll}7 & 0 & 1.637776 & 0.219971 & -0.077187\end{array}$

$\begin{array}{llllll}6 & 0 & 2.296049 & -0.524704 & 1.008403\end{array}$

$\begin{array}{llllll}6 & 0 & 1.497334 & -0.736850 & -1.178670\end{array}$

$\begin{array}{llllll}1 & 0 & 2.232868 & 0.984081 & -0.375235\end{array}$

$\begin{array}{llllll}6 & 0 & -0.379061 & 1.213917 & 0.684417\end{array}$

$\begin{array}{llllll}6 & 0 & 3.112656 & -1.664009 & 0.349717\end{array}$

$\begin{array}{lllll}1 & 0 & 1.522790 & -0.943452 & 1.657923\end{array}$

$\begin{array}{llllll}1 & 0 & 2.901935 & 0.152350 & 1.608365\end{array}$

$\begin{array}{llllll}1 & 0 & 0.631906 & -1.372859 & -0.971318\end{array}$

$\begin{array}{llllll}1 & 0 & 1.317686 & -0.215956 & -2.118034\end{array}$

$\begin{array}{llllll}6 & 0 & 2.783579 & -1.563531 & -1.151585\end{array}$

$\begin{array}{lllll}1 & 0 & 0.126538 & 1.187329 & 1.638341\end{array}$

$\begin{array}{llllll}6 & 0 & -1.219402 & 0.070241 & 0.363649\end{array}$

$\begin{array}{llllll}7 & 0 & -0.394976 & 2.401186 & 0.155442\end{array}$

$\begin{array}{llllll}1 & 0 & 4.180045 & -1.549847 & 0.534307\end{array}$

$\begin{array}{llllll}1 & 0 & 2.809866 & -2.629307 & 0.755628\end{array}$

$\begin{array}{llllll}1 & 0 & 2.662331 & -2.537829 & -1.624112\end{array}$

$\begin{array}{llllll}1 & 0 & 3.574012 & -1.028473 & -1.681689\end{array}$

$\begin{array}{lllll}6 & 0 & -1.340916 & -0.890722 & 1.373866\end{array}$

$\begin{array}{llllll}6 & 0 & -1.846642 & -0.148015 & -0.867860\end{array}$

$\begin{array}{llllll}6 & 0 & 0.465144 & 3.495852 & 0.575698\end{array}$

$\begin{array}{llllll}8 & 0 & -0.968604 & 2.590924 & -1.080247\end{array}$

$\begin{array}{llllll}6 & 0 & -2.084777 & -2.039102 & 1.166134\end{array}$

$\begin{array}{llllll}1 & 0 & -0.856976 & -0.732816 & 2.330039\end{array}$

$\begin{array}{llllll}6 & 0 & -2.585874 & -1.300248 & -1.067662\end{array}$

$\begin{array}{llllll}1 & 0 & -1.740345 & 0.567941 & -1.666514\end{array}$

$\begin{array}{llllll}1 & 0 & 1.264033 & 3.618620 & -0.154991\end{array}$

$\begin{array}{llllll}1 & 0 & -0.126361 & 4.407539 & 0.632693\end{array}$

$\begin{array}{llllll}1 & 0 & 0.880625 & 3.266130 & 1.552717\end{array}$ 


$\begin{array}{lllll}1 & 0 & -1.754845 & 3.131945 & -0.926148 \\ 6 & 0 & -2.707266 & -2.245449 & -0.056839 \\ 1 & 0 & -2.173810 & -2.773304 & 1.955908 \\ 1 & 0 & -3.064374 & -1.465483 & -2.024180 \\ 1 & 0 & -3.283635 & -3.146378 & -0.225119\end{array}$

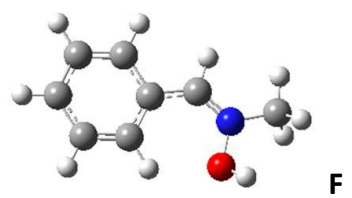

$E(w B 97 x d /$ tzvp $)=-440.616168384$

$\mathrm{H}$ (correction) $=0.179601$

$\mathrm{G}($ correction $)=0.134499$

Imaginary frequencies: 0

$\begin{array}{rrrrr}6 & 0 & -1.017490 & -0.724642 & -0.005602 \\ 1 & 0 & -1.194065 & -1.792911 & -0.018190 \\ 6 & 0 & 0.356410 & -0.292444 & -0.011699 \\ 7 & 0 & -2.106798 & -0.040754 & 0.016664 \\ 6 & 0 & 1.300089 & -1.333014 & -0.001663 \\ 6 & 0 & 0.801170 & 1.039790 & -0.014262 \\ 8 & 0 & -2.049504 & 1.318273 & -0.078456 \\ 6 & 0 & -3.453797 & -0.606013 & 0.000149 \\ 6 & 0 & 2.652468 & -1.053139 & 0.010729 \\ 1 & 0 & 0.965467 & -2.363232 & -0.002033 \\ 6 & 0 & 2.156544 & 1.306214 & 0.000471 \\ 1 & 0 & 0.095043 & 1.853147 & -0.030150 \\ 1 & 0 & -2.666880 & 1.668182 & 0.579359 \\ 1 & 0 & -3.982352 & -0.271884 & 0.891856 \\ 1 & 0 & -3.957734 & -0.254495 & -0.897193 \\ 1 & 0 & -3.381474 & -1.688650 & 0.001236 \\ 6 & 0 & 3.080657 & 0.267730 & 0.012827 \\ 1 & 0 & 3.371686 & -1.861012 & 0.018481 \\ 1 & 0 & 2.497355 & 2.333117 & -0.000616 \\ 1 & 0 & 4.140264 & 0.489930 & 0.022540\end{array}$




\section{NMR SPECTRA $\left(\mathrm{CDCl}_{3}\right)$}

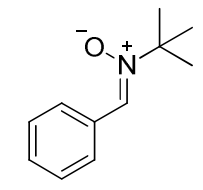

(Z)-N-Benzylidene-2-methylpropan-2-amine oxide
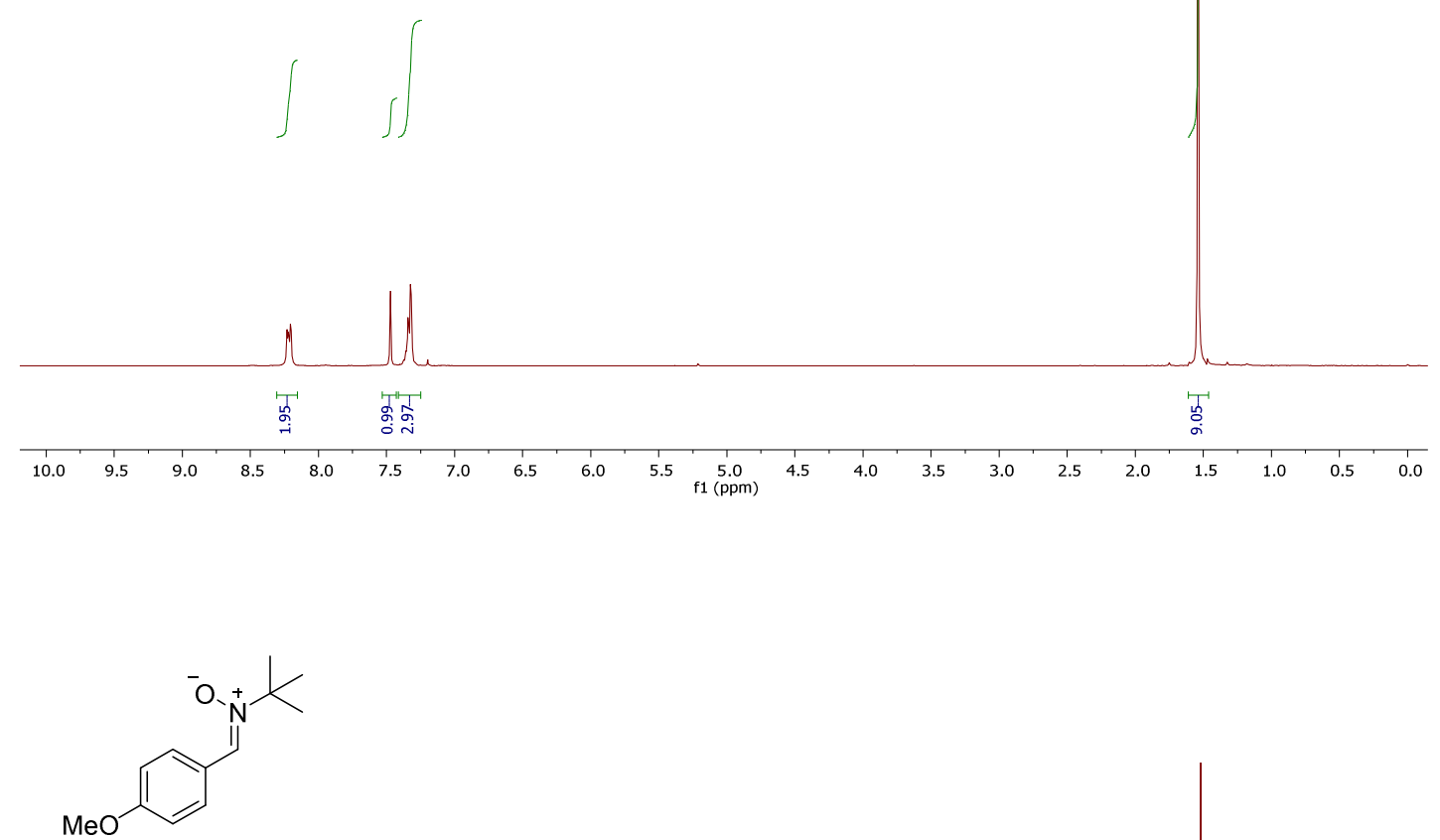

(Z)-N-(4-Methoxybenzylidene)-2-methylpropan-2-amine oxide

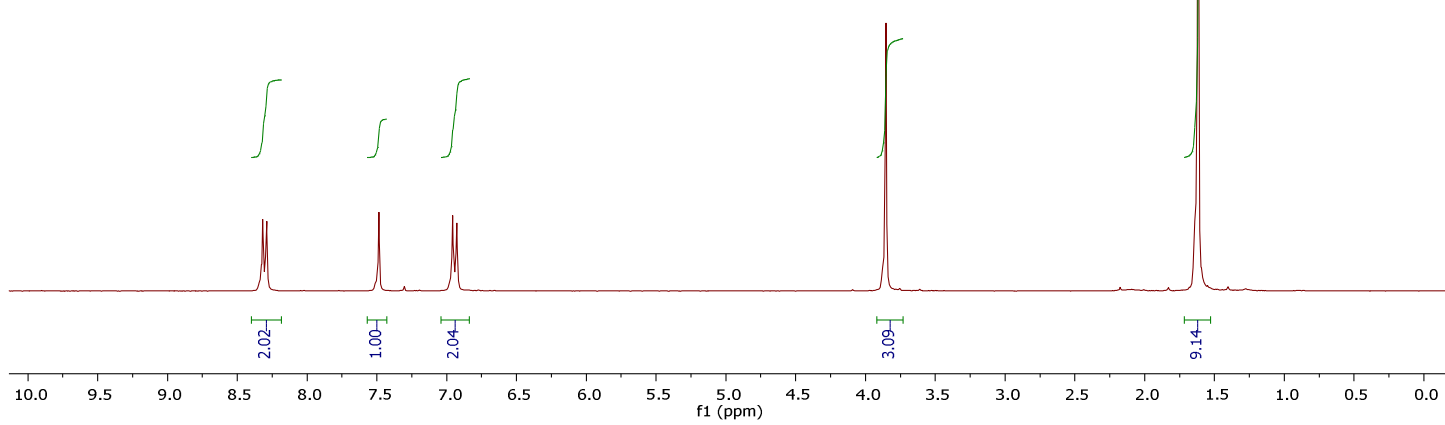




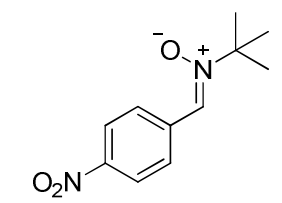

(Z)-N-(4-Nitrobenzylidene)2-methylpropan-2-amine oxide
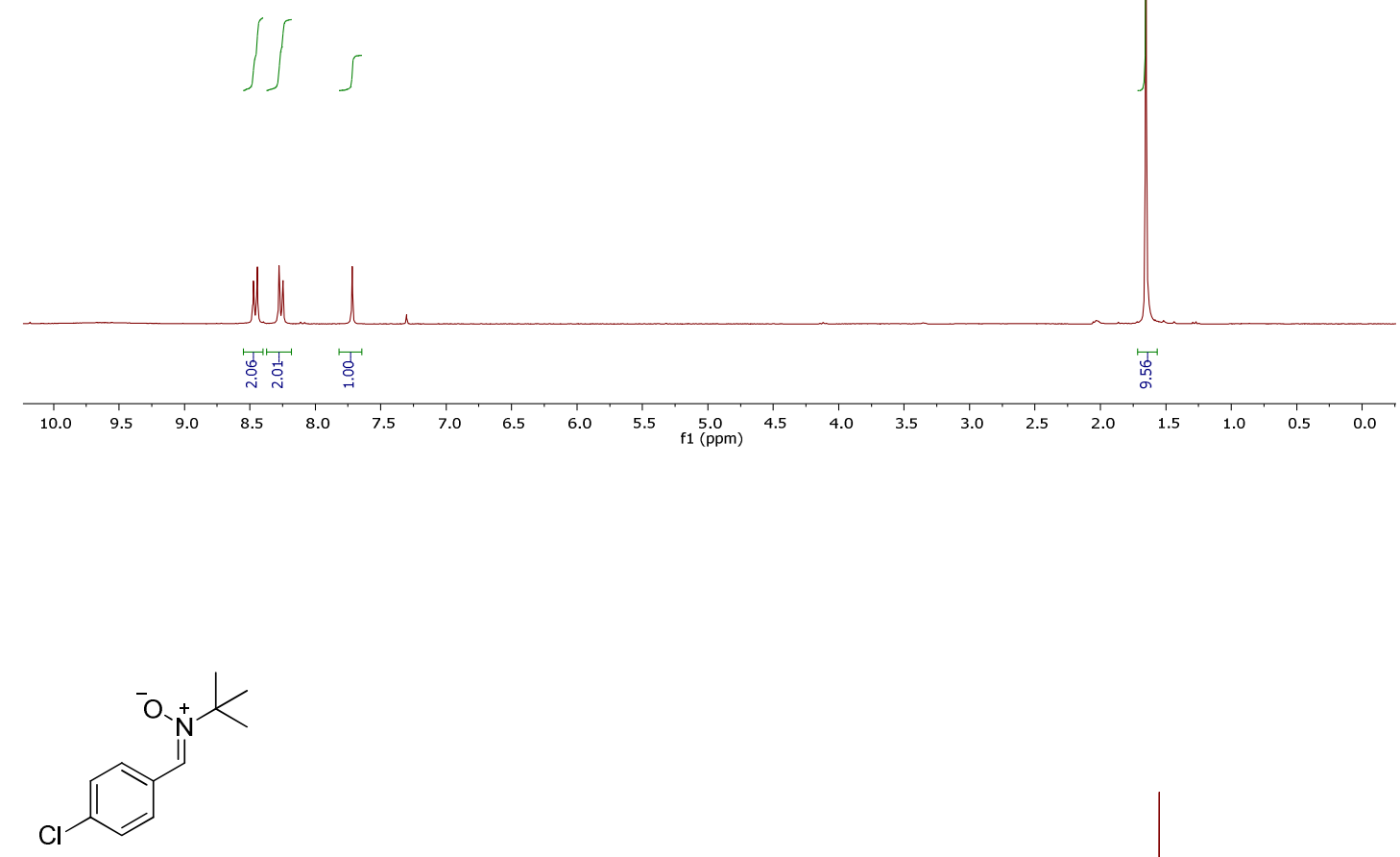

(Z)-N-(4-Chlorobenzylidene)-2-methylpropan-2-amine oxide

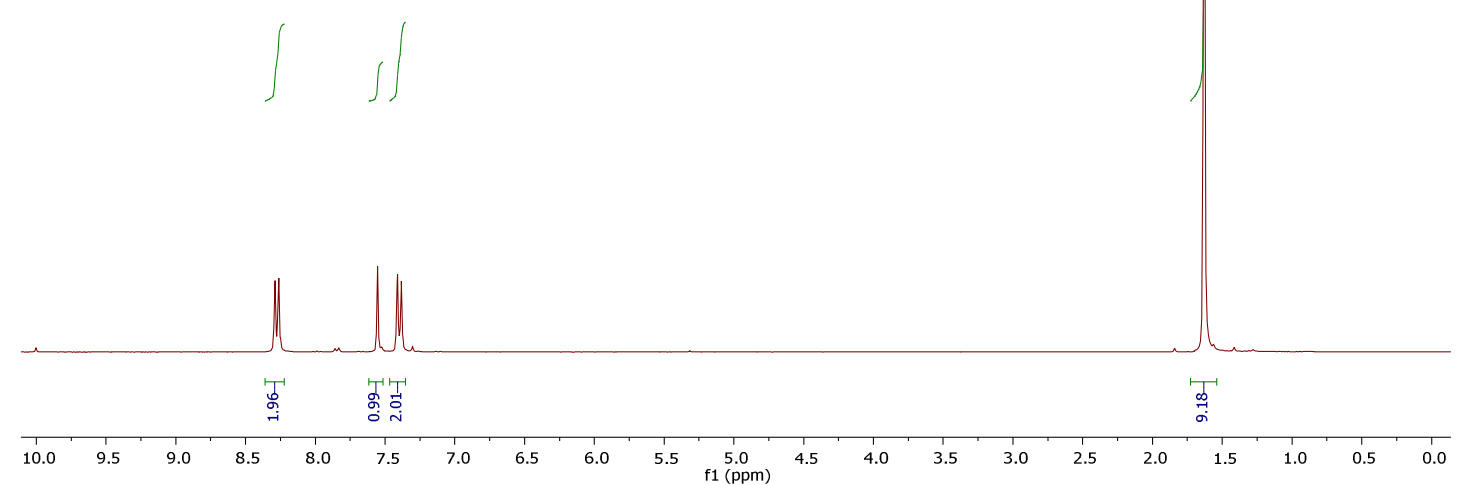




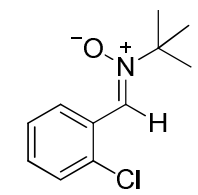

(Z)-N-(2-Chlorobenzylidene)-2-methylpropan-2-amine oxide
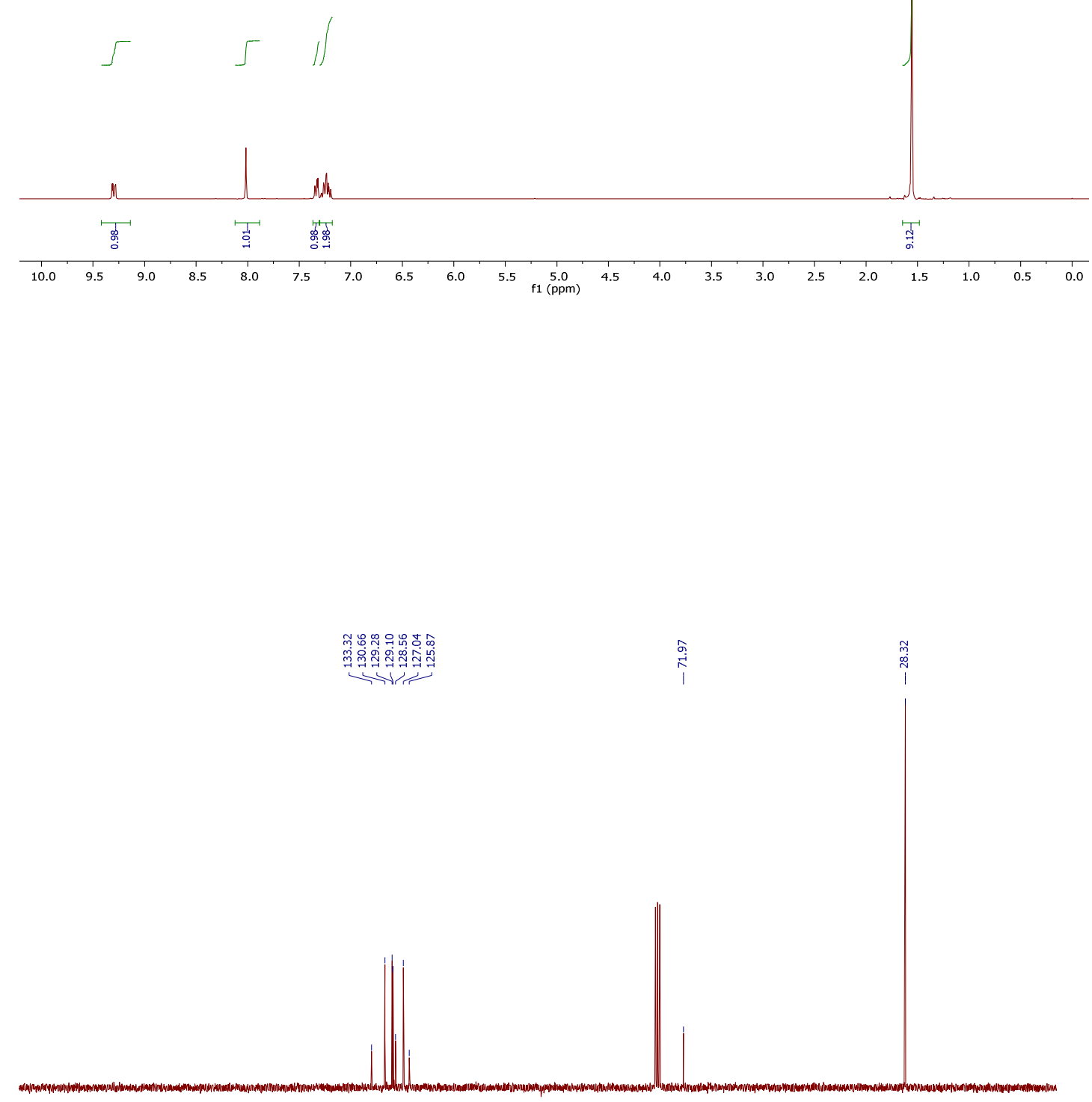


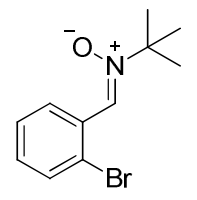

(Z)-N-(2-Bromobenzylidene)-2-methylpropan-2-amine oxide
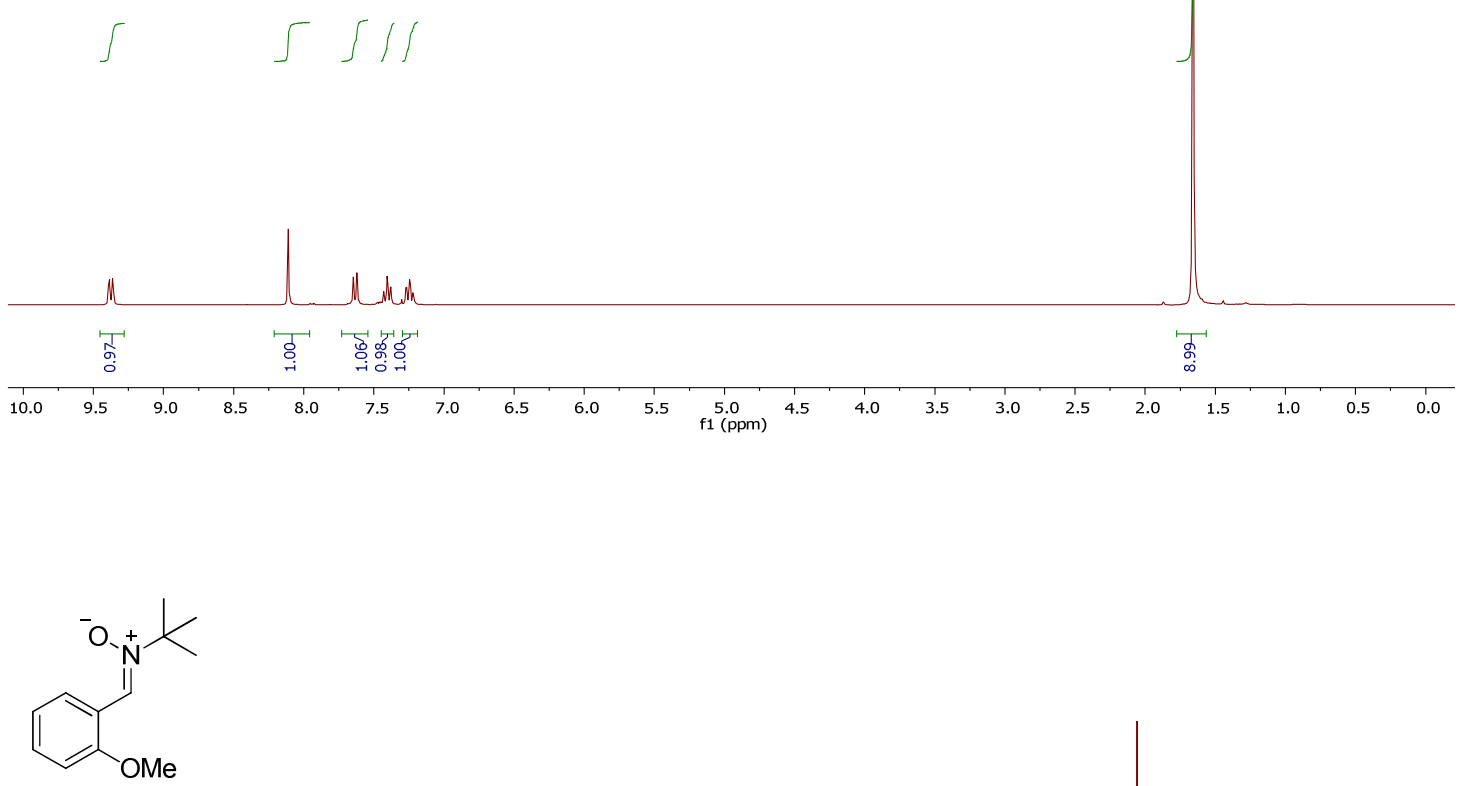

(Z)-N-(2-Methoxybenzylidene)-2-methylpropan-2-amine oxide

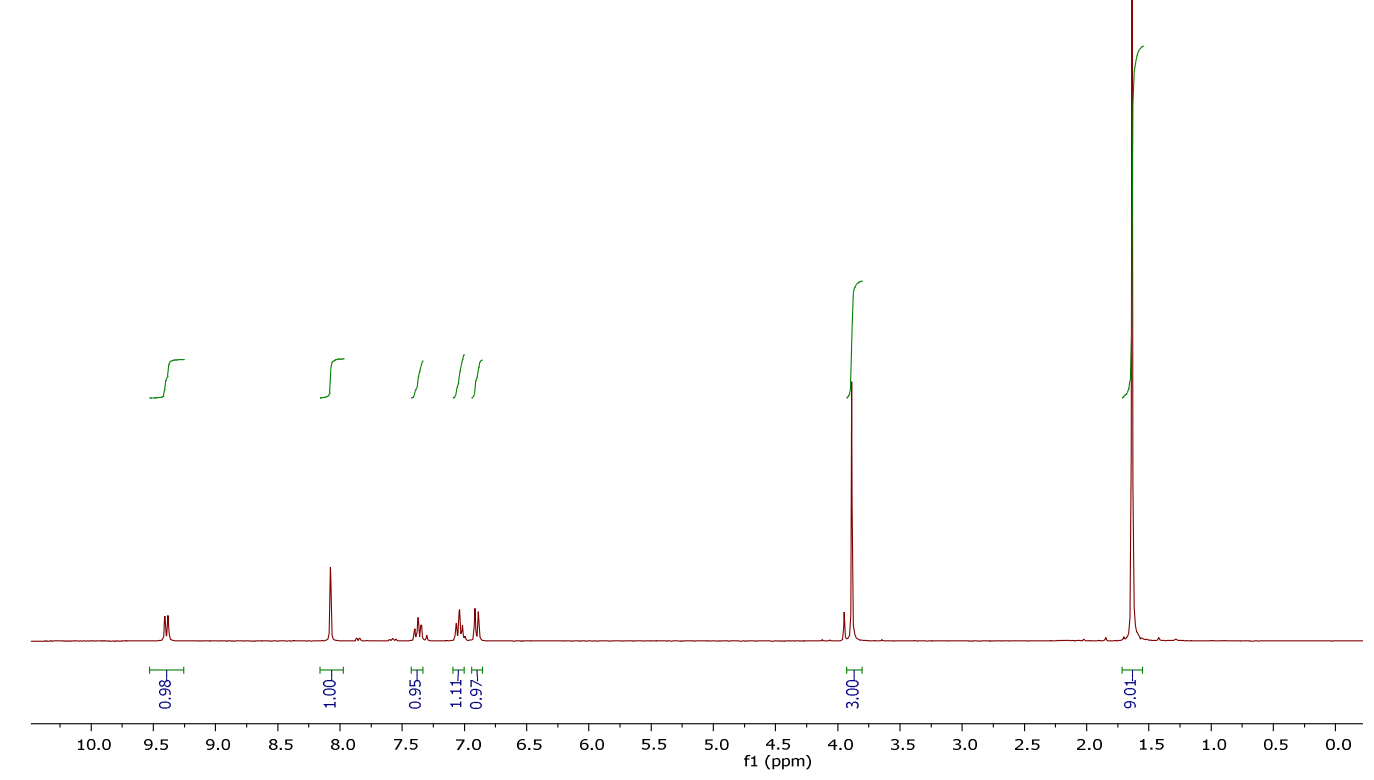




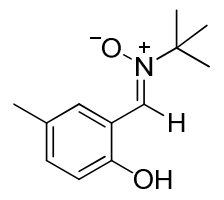

(Z)-N-(2-Hydroxy-5-methylbenzylidene)-2-methylpropan-2-amine oxide

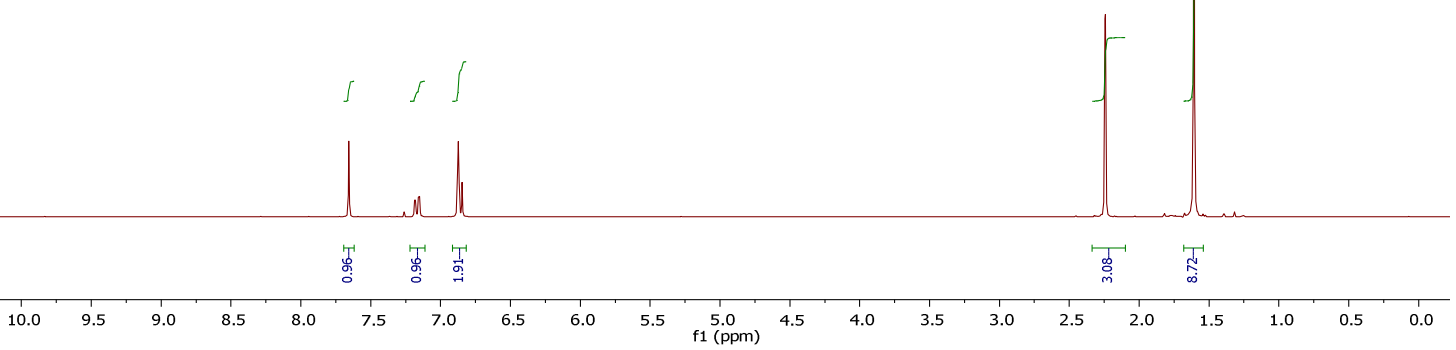

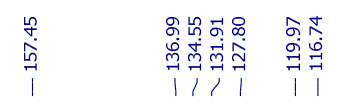

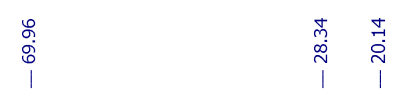
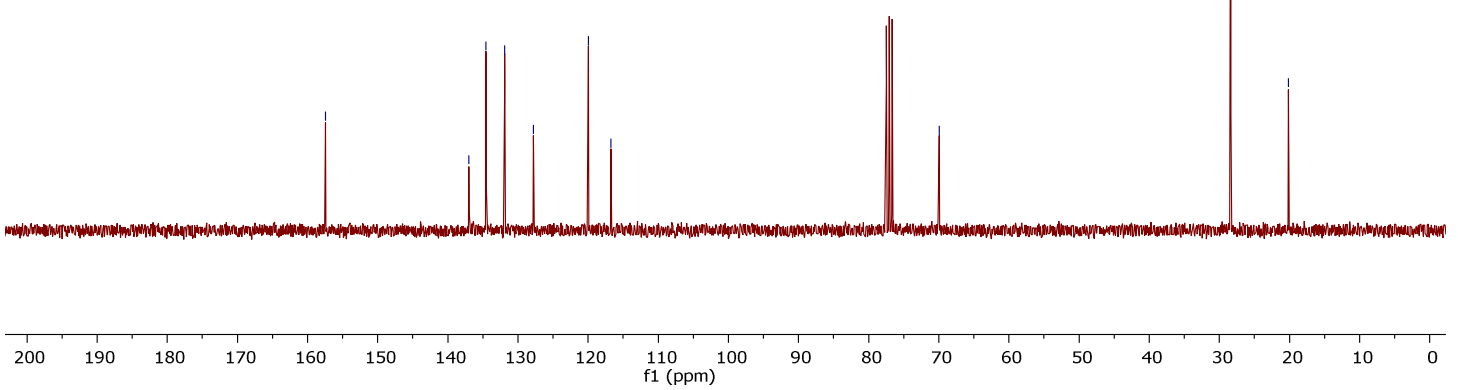

S35 


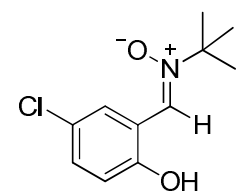

(Z)-N-(5-Chloro-2-hydroxybenzylidene)-2-methylpropan-2-amine oxide

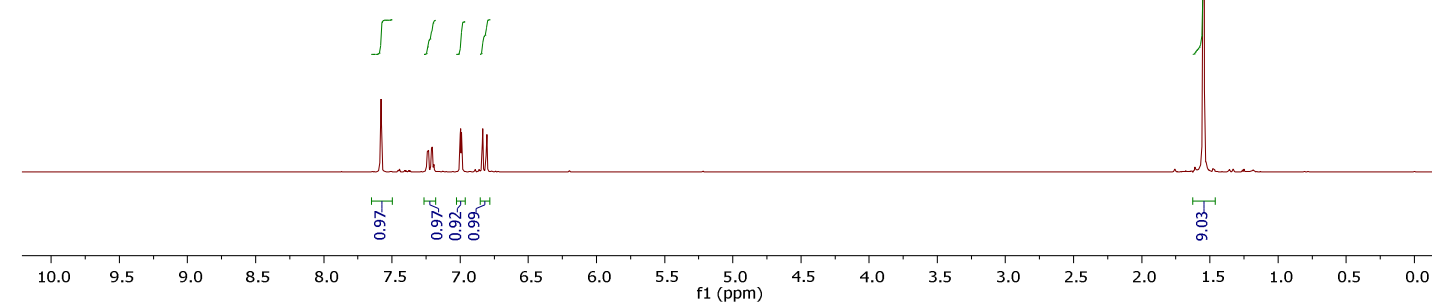

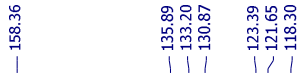

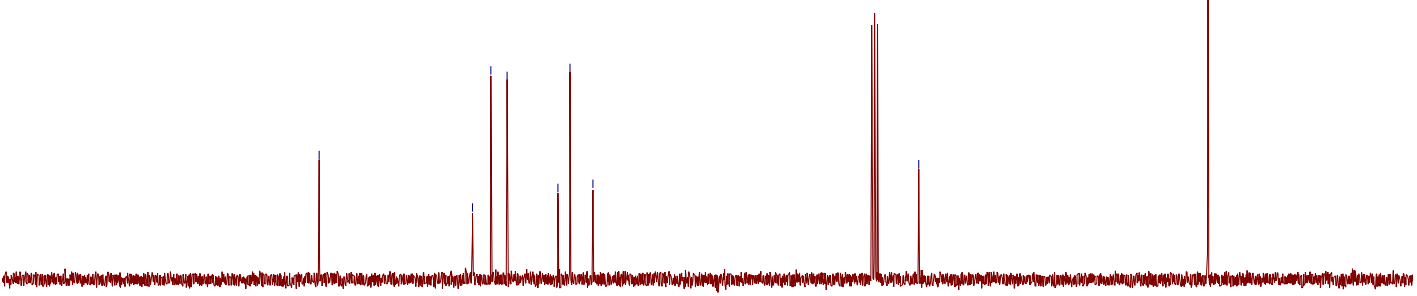




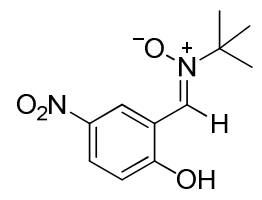

(Z)-N-(2-Hydroxy-5-nitrobenzylidene)-2-methylpropan-2-amine oxide
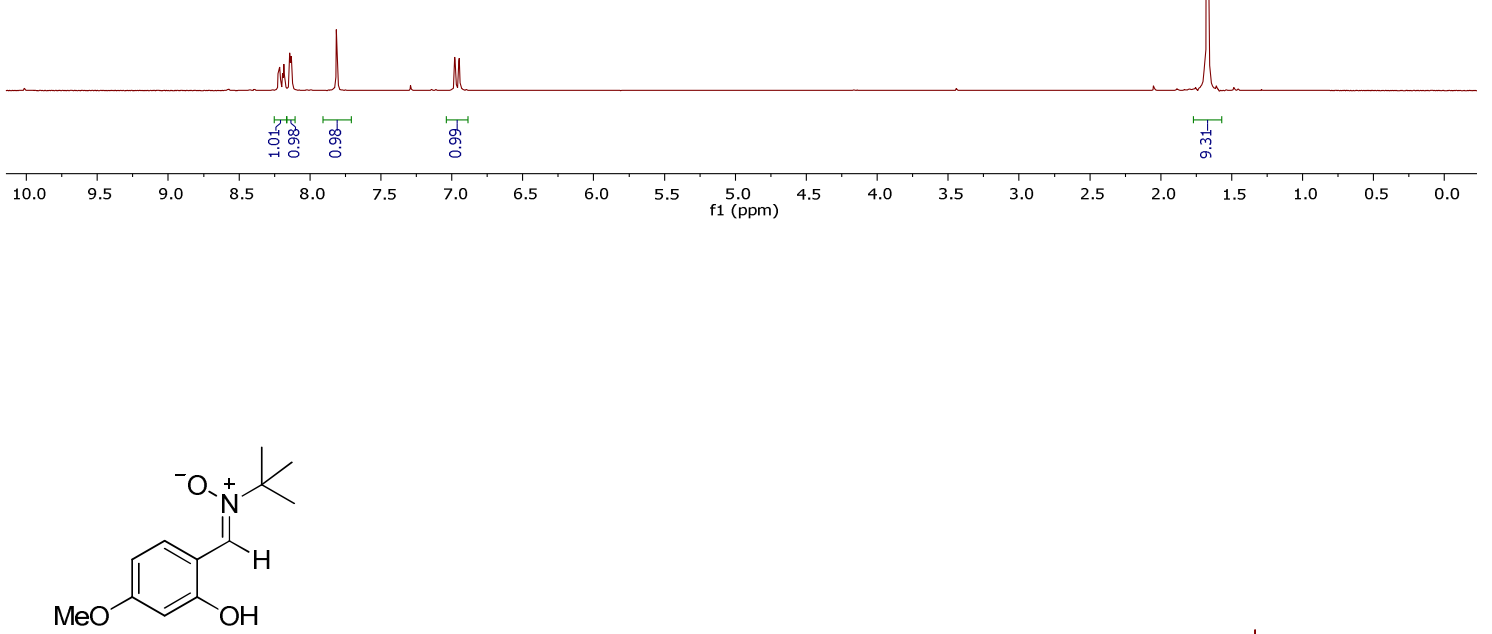

(Z)-N-(2-Hydroxy-4-methoxybenzylidene)-2-methylpropan-2-amine oxide

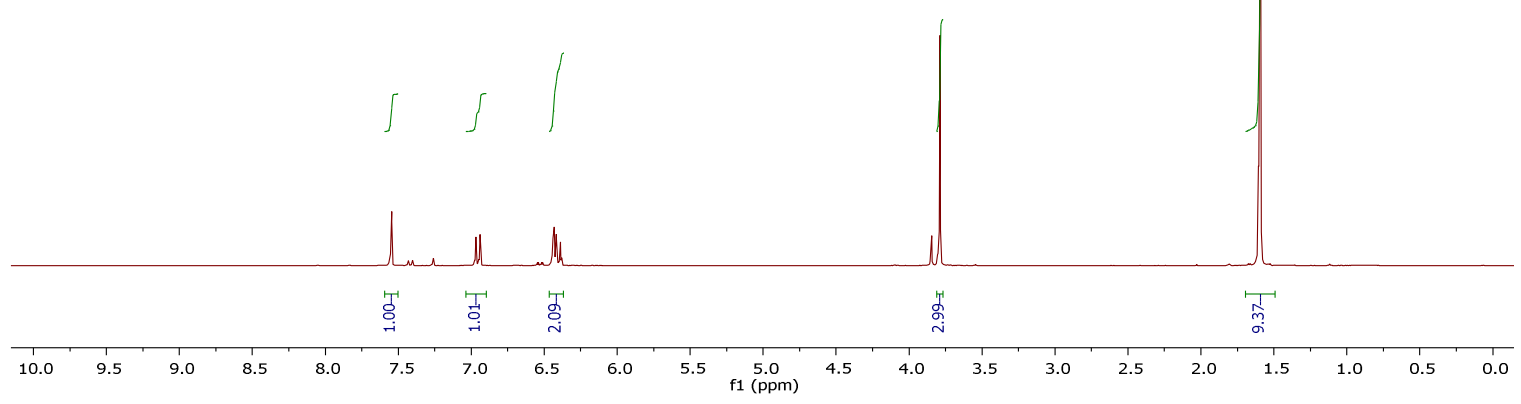

S37 


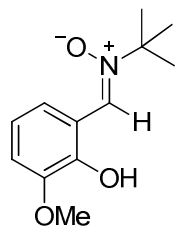

(Z)-N-(2-Hydroxy-3-methoxybenzylidene)-2-methylpropan-2-amine oxide

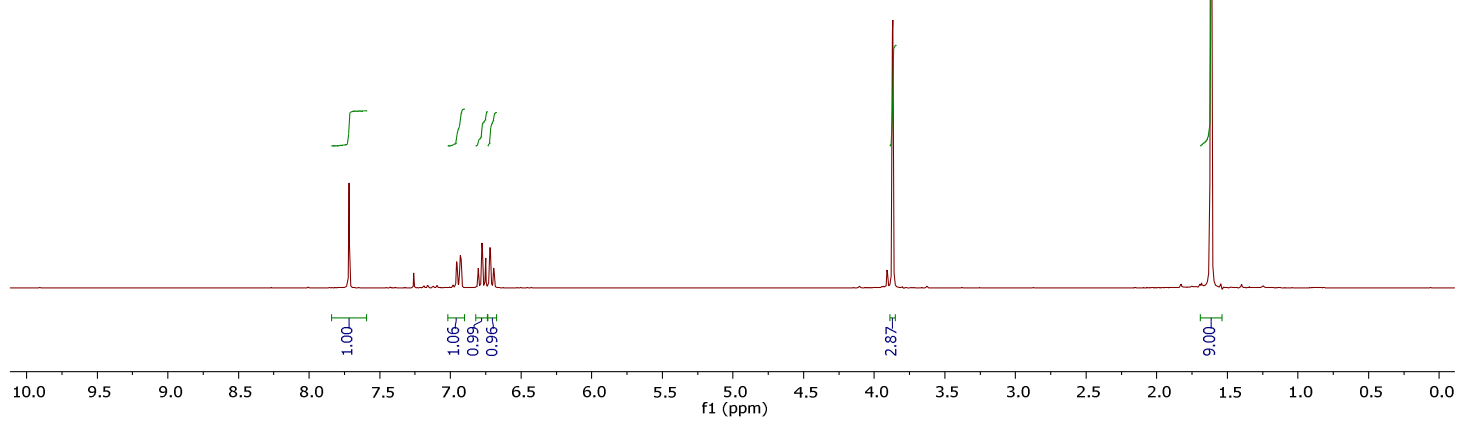

|

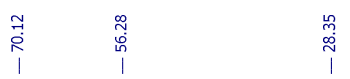

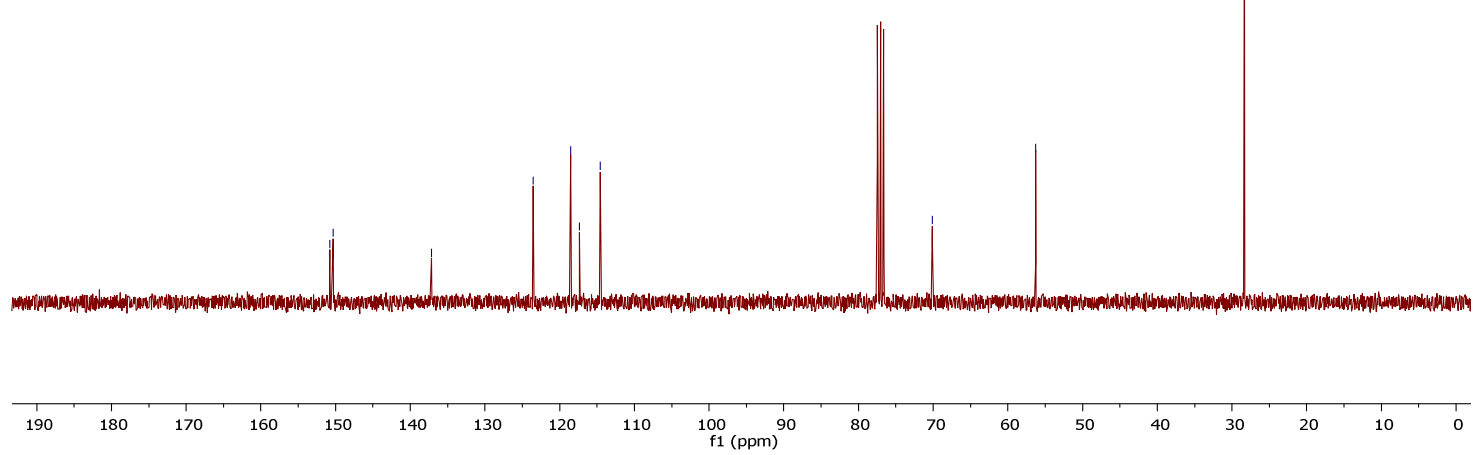

S38 


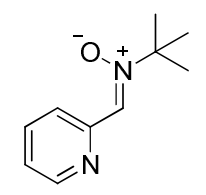

(Z)-N-(Pyridin-2-ylmethylene)-2-methylpropan-2-amine oxide
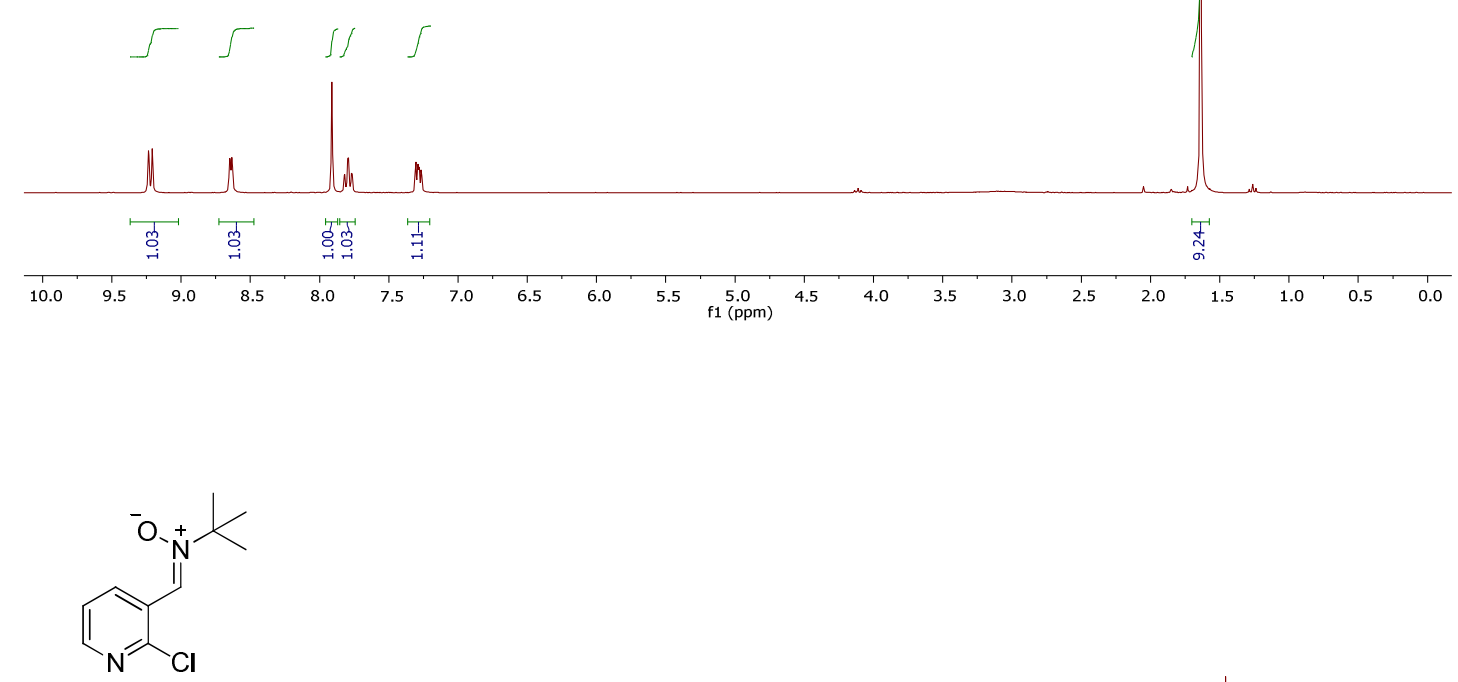

\section{(Z)-N-(2-Chloropyridin-3-yl)methylene)-2-methylpropan-2-amine oxide}

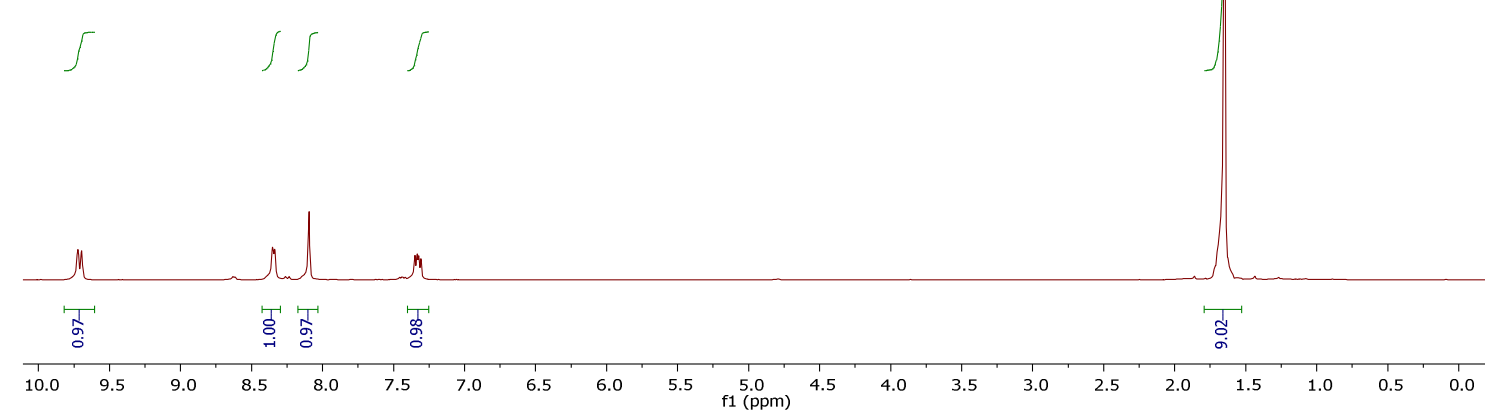




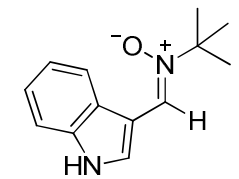

(Z)-N-(1H-Indol-3-yl)methylene)-2-methylpropan-2-amine oxide

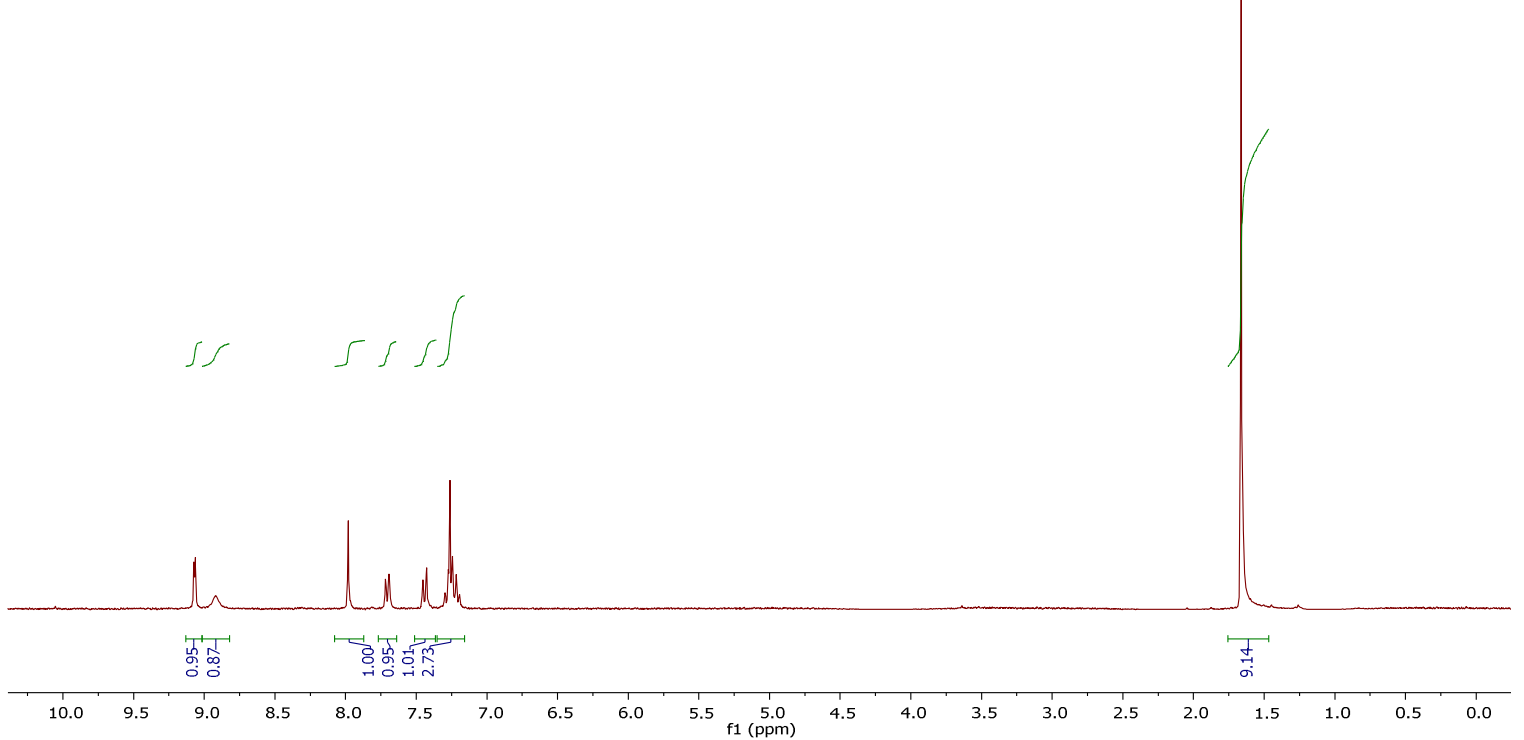




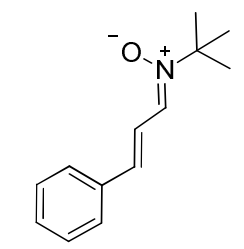

(Z)-N-((E)-3-Phenylallylidene)-2-methylpropan-2-amine oxide
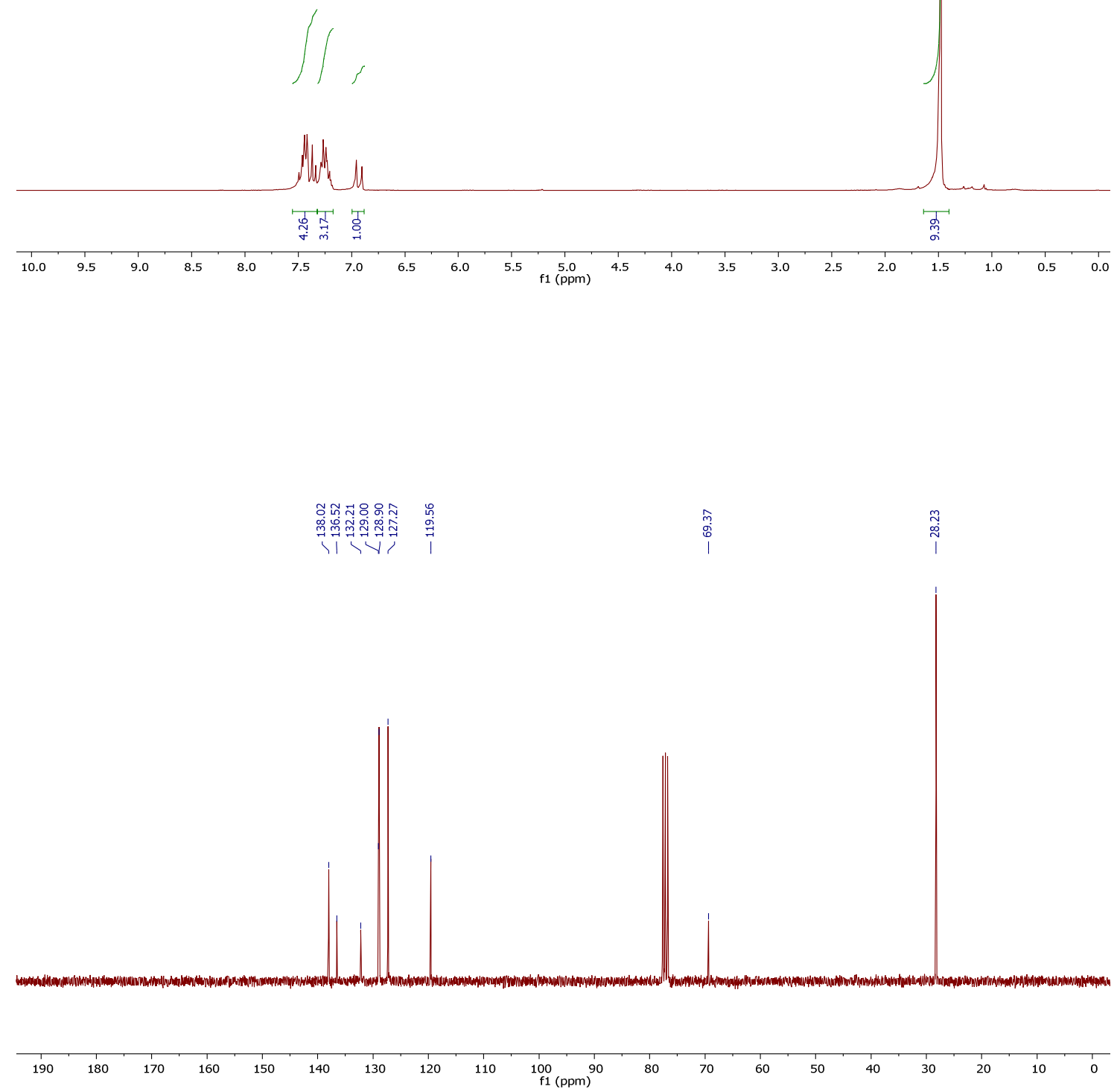


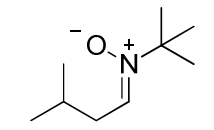

(Z)-N-(3-Methylbutylidene)-2-methylpropan-2-amine oxide
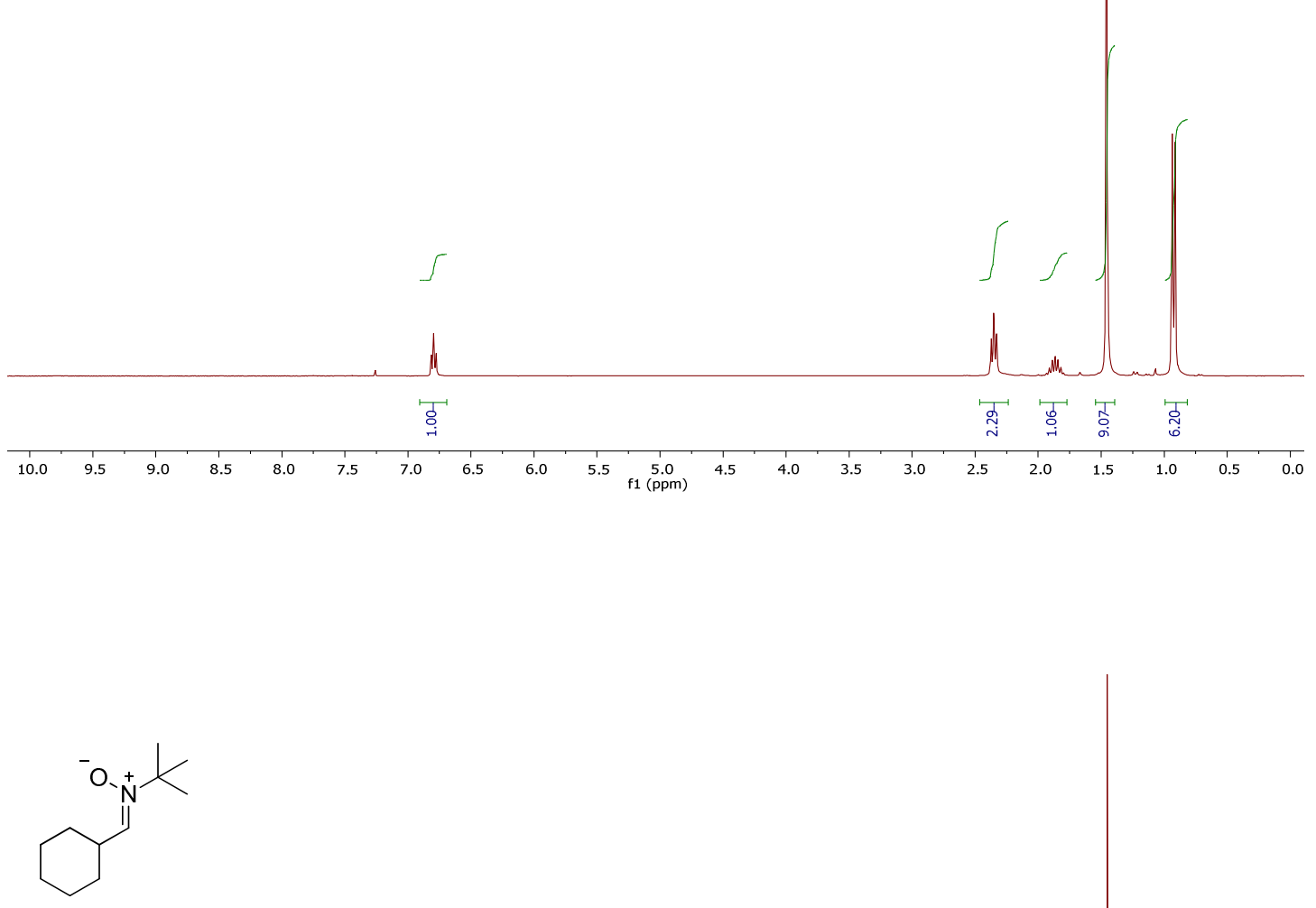

(Z)-N-(Cyclohexylmethylene)-2-methylpropan-2-amine oxide

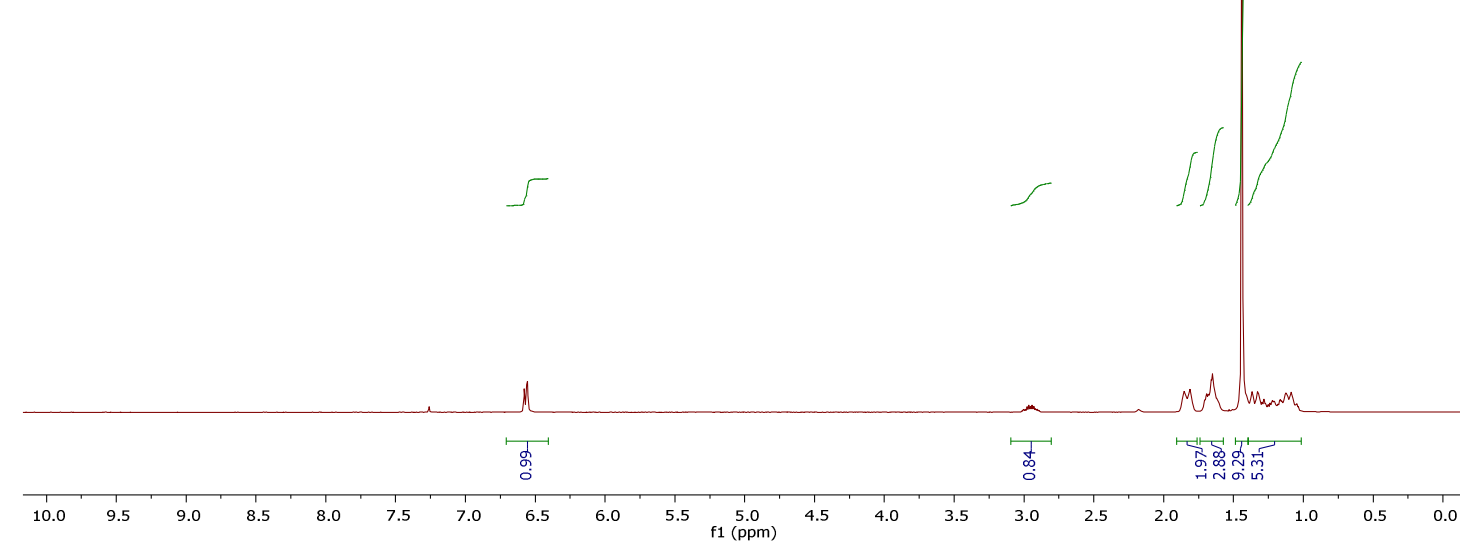




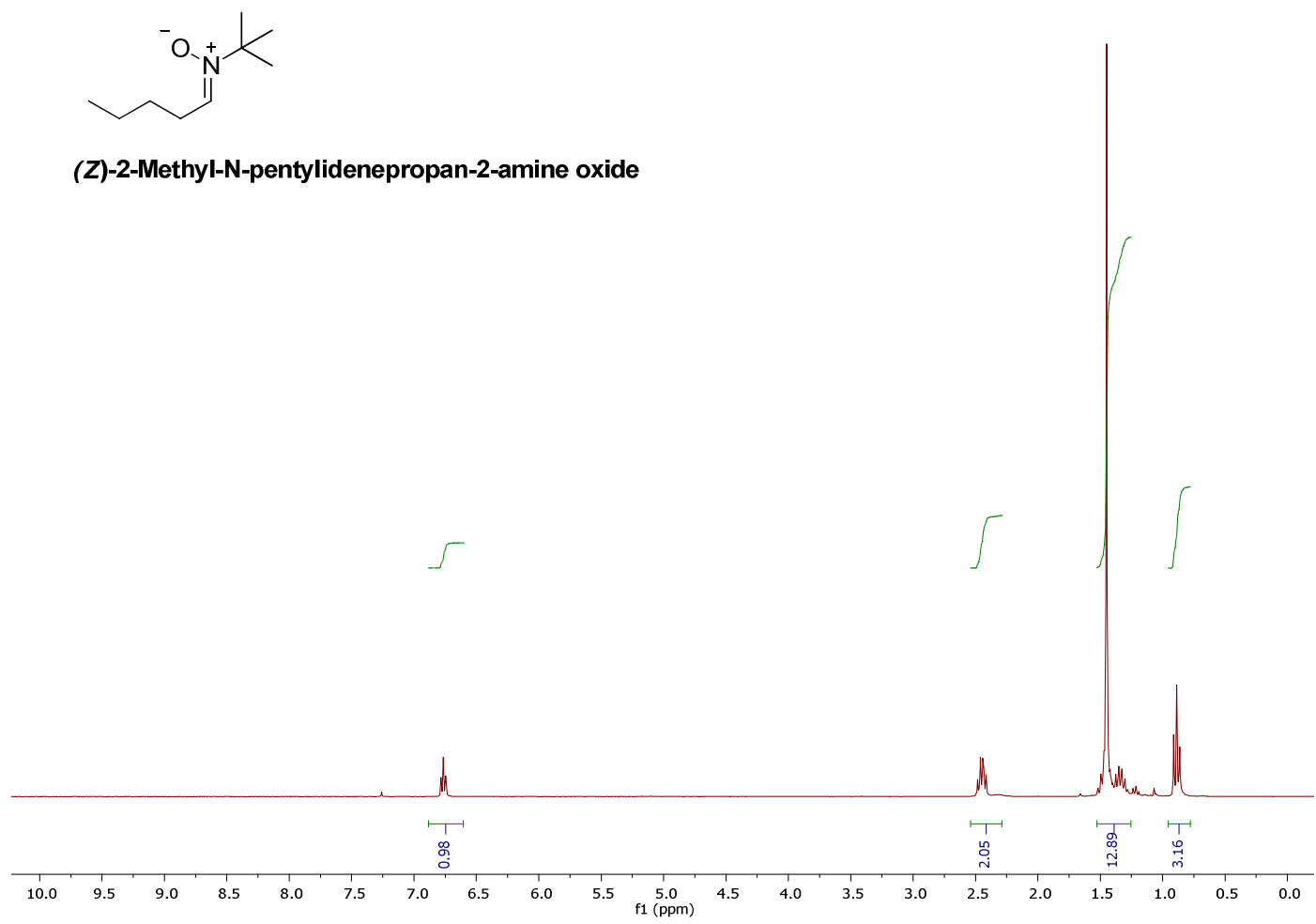

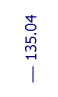

ภ
0
0
1

证

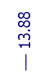

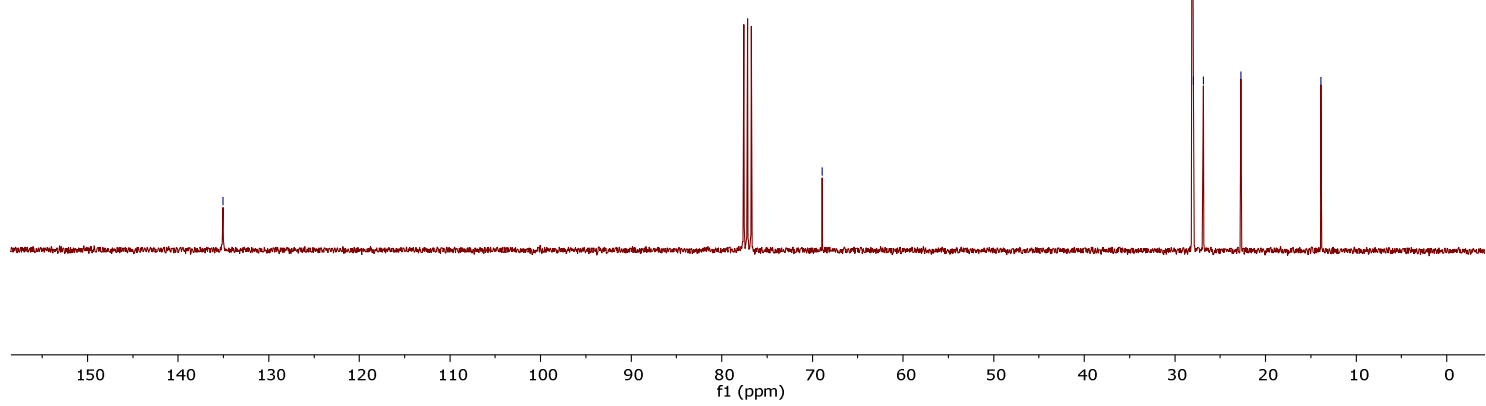

S43 


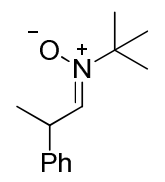

(Z)-N-(2-Phenylpropylidene)-2-methylpropan-2-amine oxide
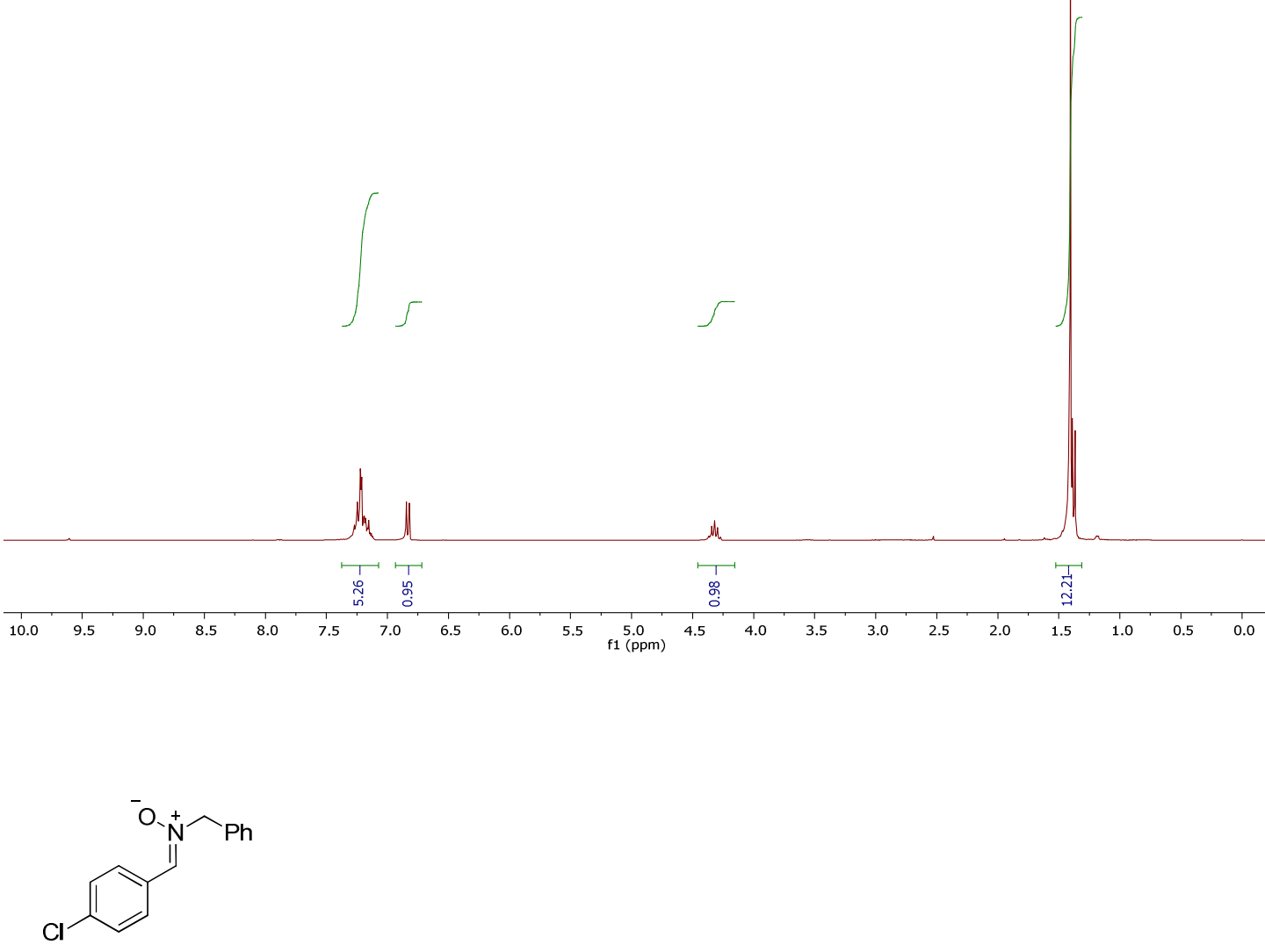

(Z)-N-(4-Chlorobenzylidene)-1-phenylmethanamine oxide

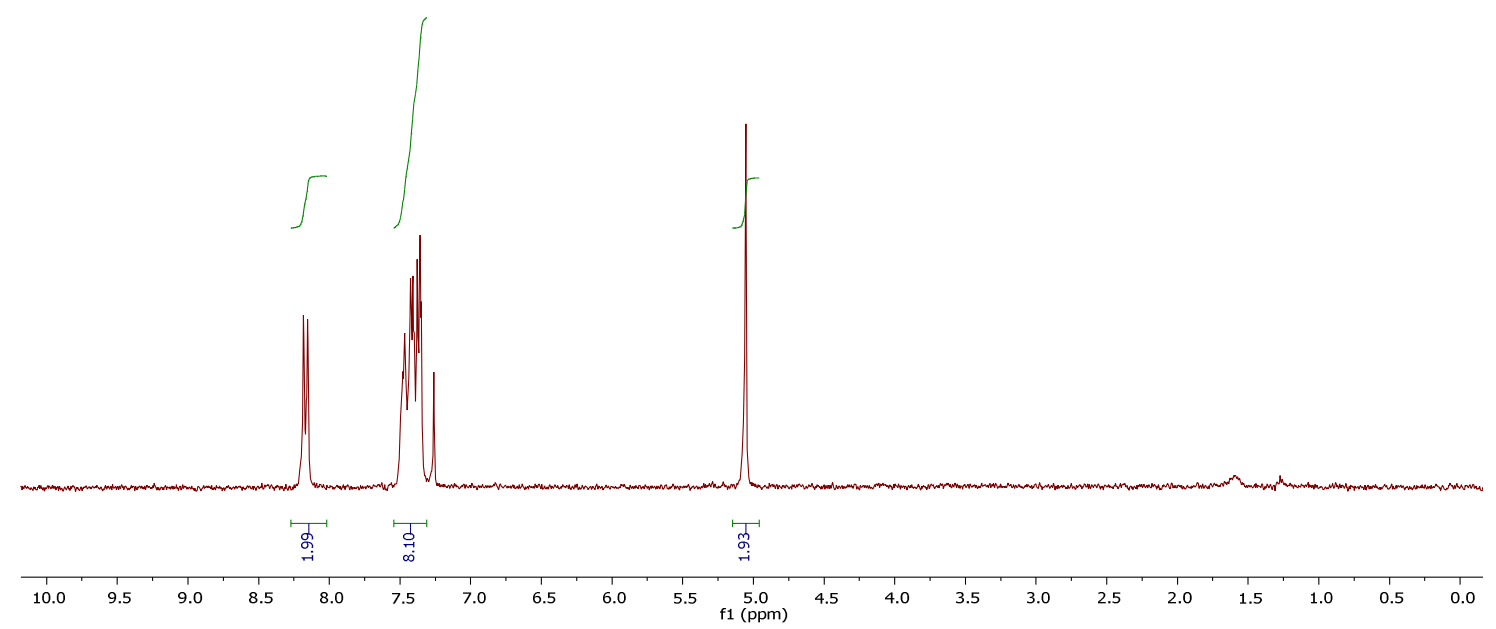




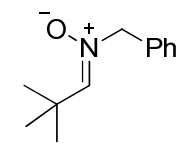

(Z)-N-(2,2-Dimethylpropylidene)-1-phenylmethanamine oxide
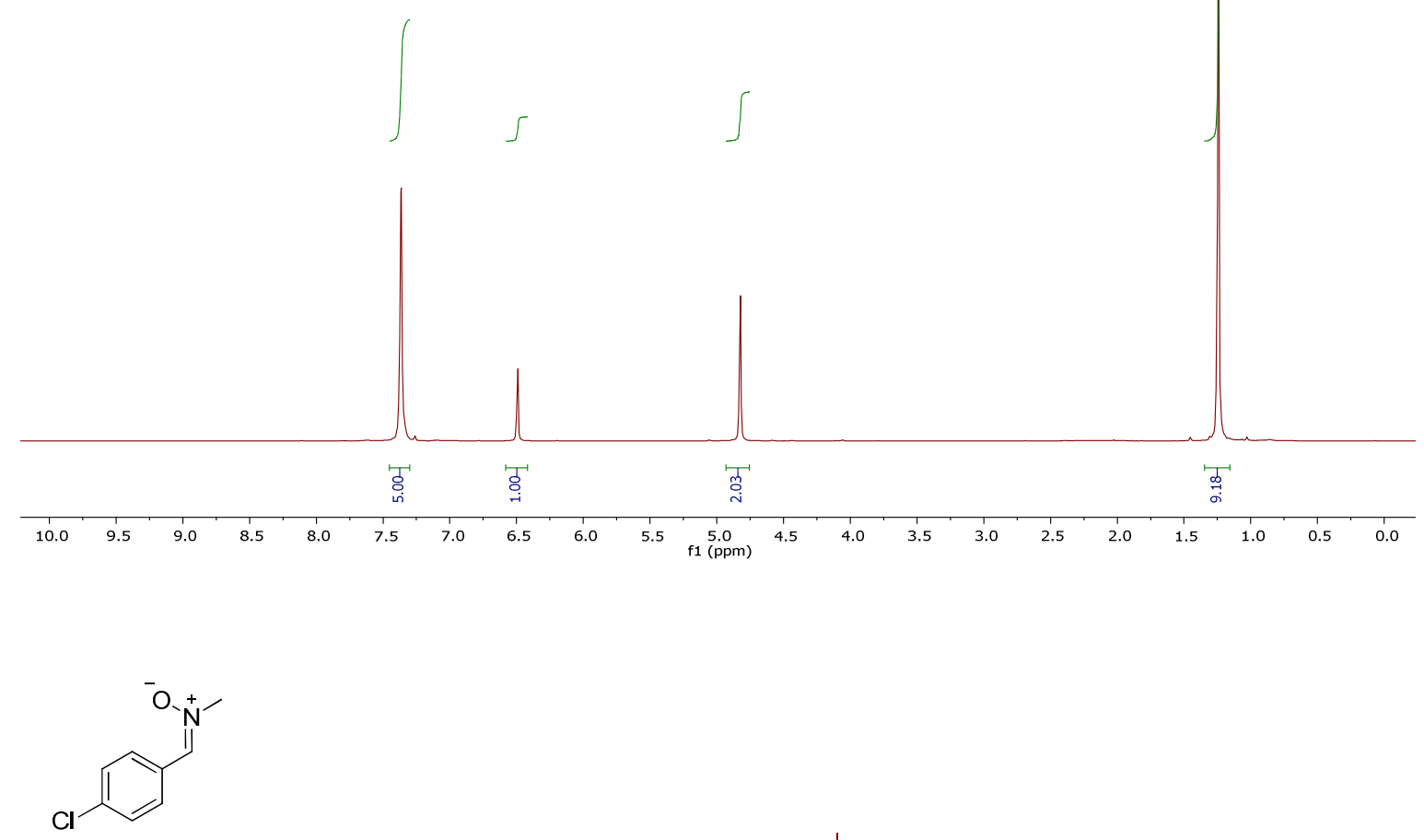

(Z)-N-(4-Chlorobenzylidene)methanamine oxide

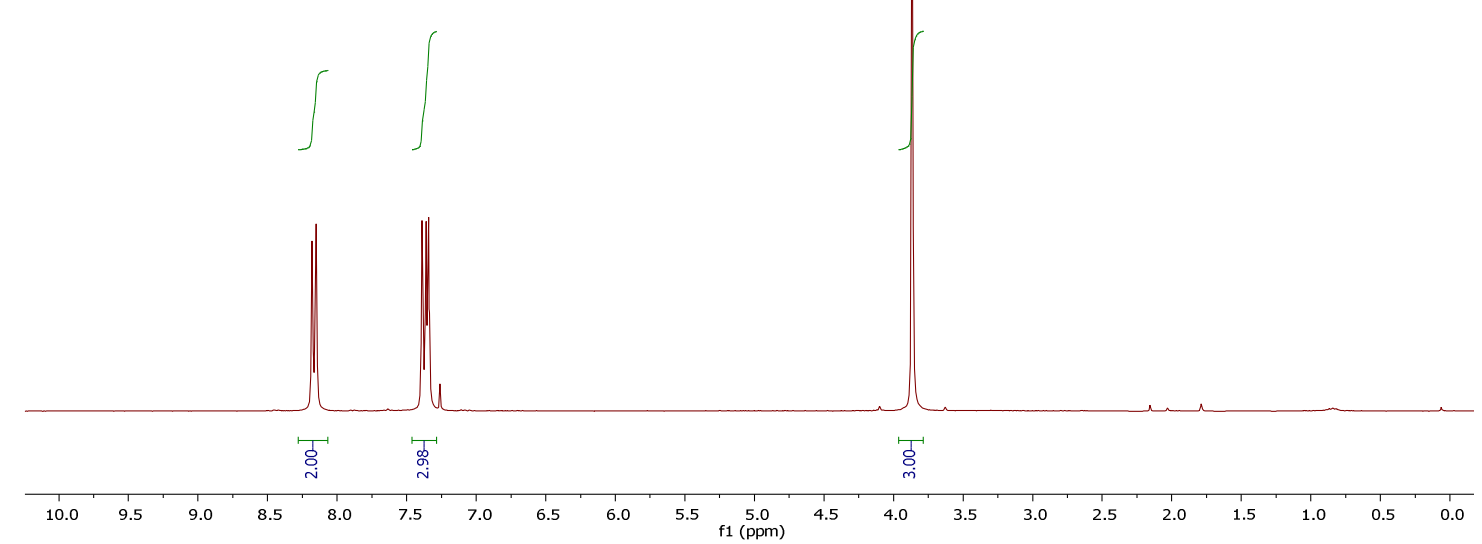




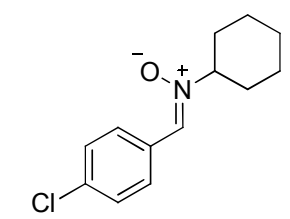

(Z)-N-(4-Chlorobenzylidene)cyclohexanamine oxide
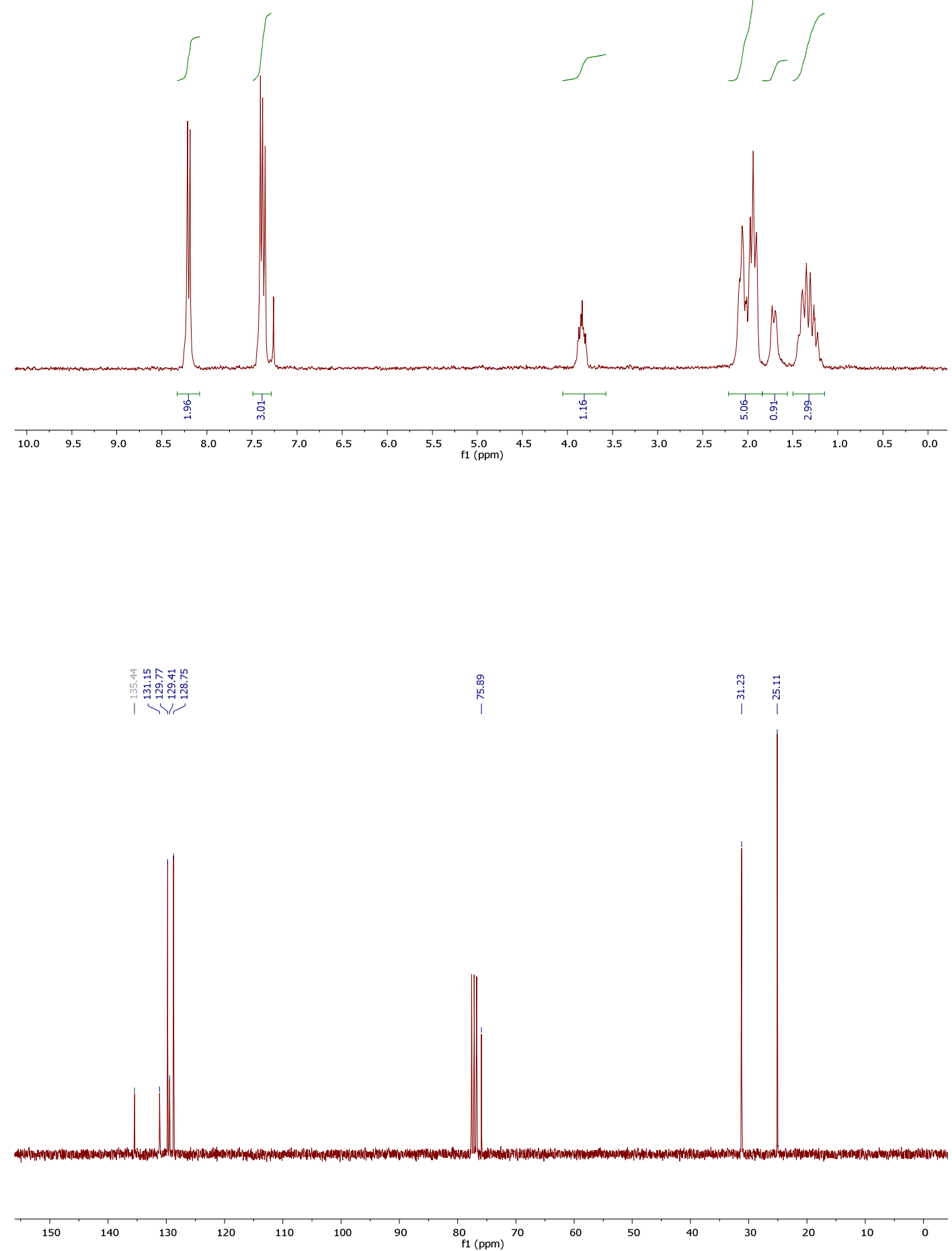


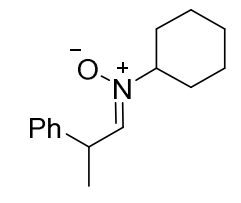

(Z)-N-cyclohexyl-2-phenylpropan-1-imine oxide
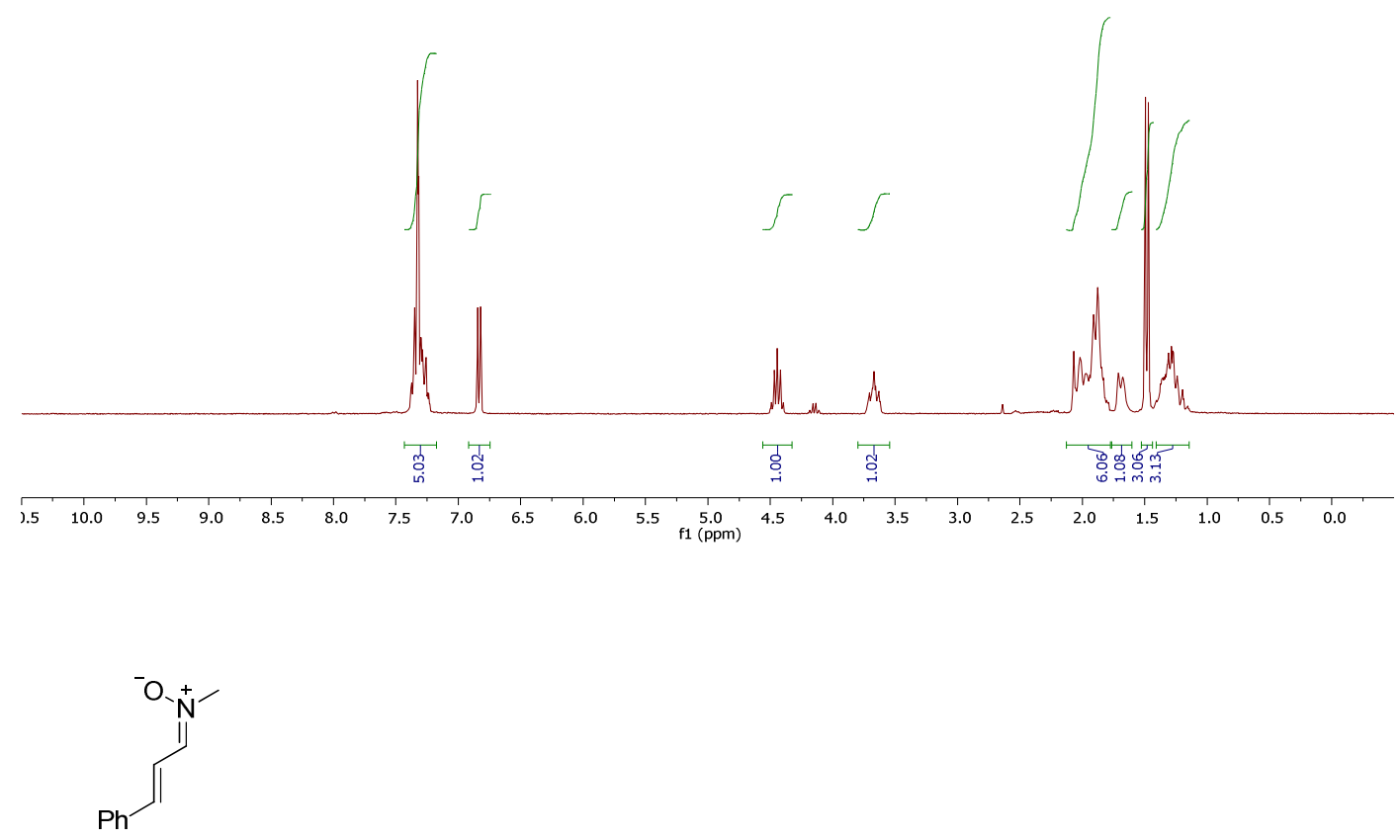

(Z)-N-((E)-3-Phenylallylidene)methanamine oxide

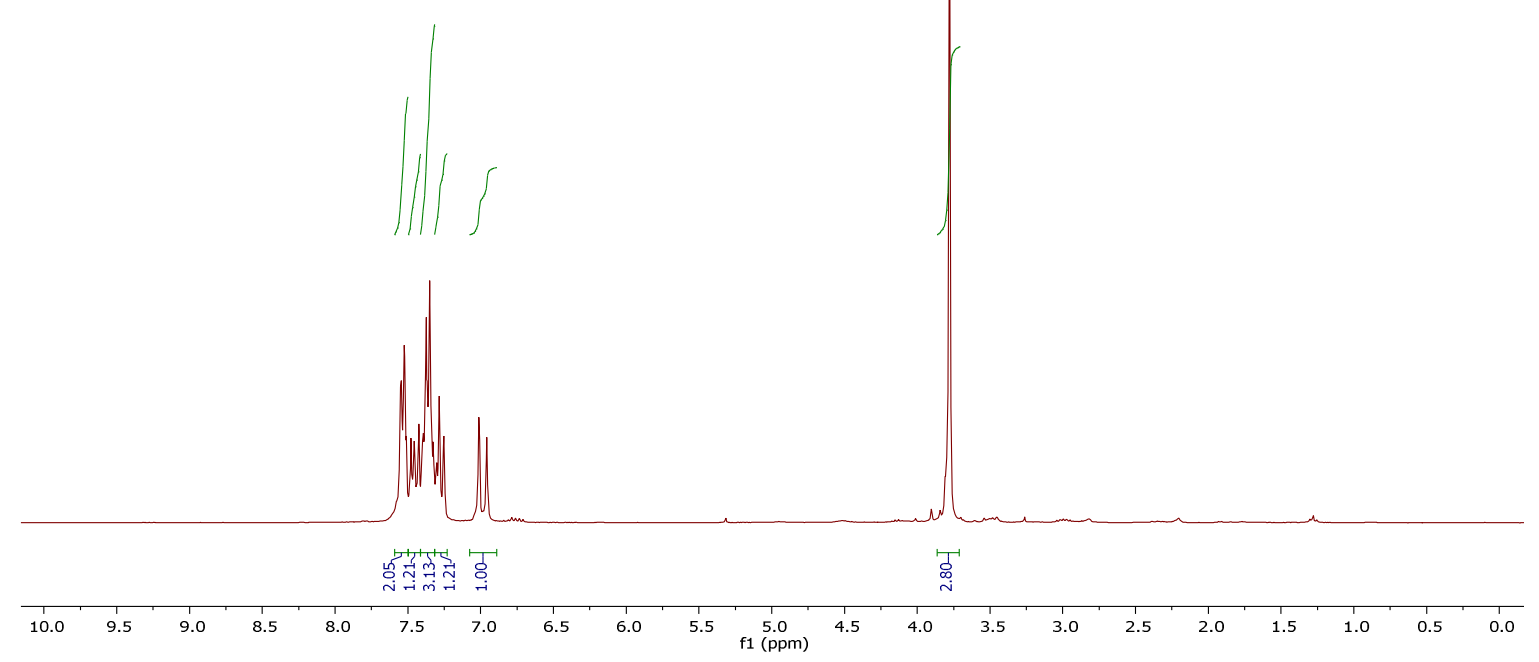




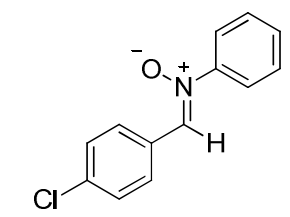

(Z)-N-(4-Chlorobenzylidene)aniline oxide

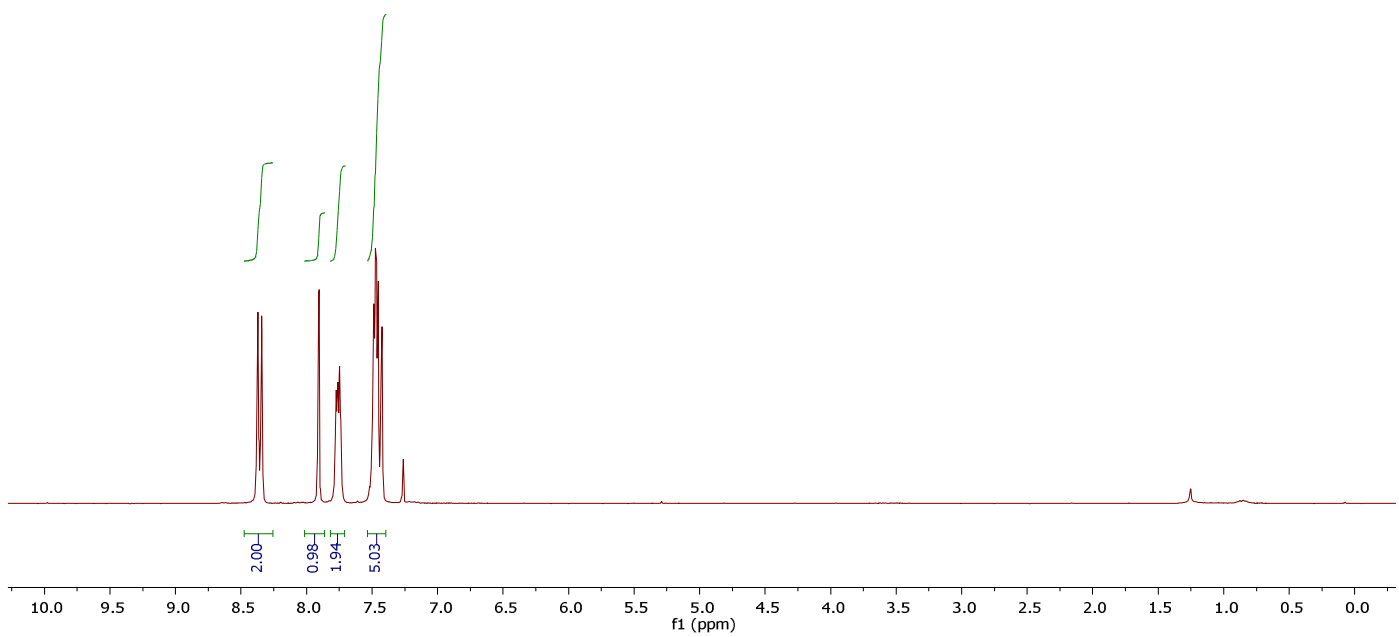

${ }^{1} \mathrm{H} \mathrm{RMN}$ in $\mathrm{D}_{2} \mathrm{O}$

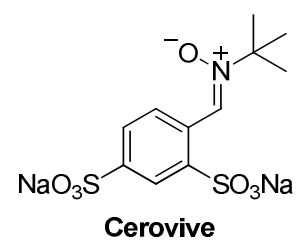

$\iint \sqrt{ }$

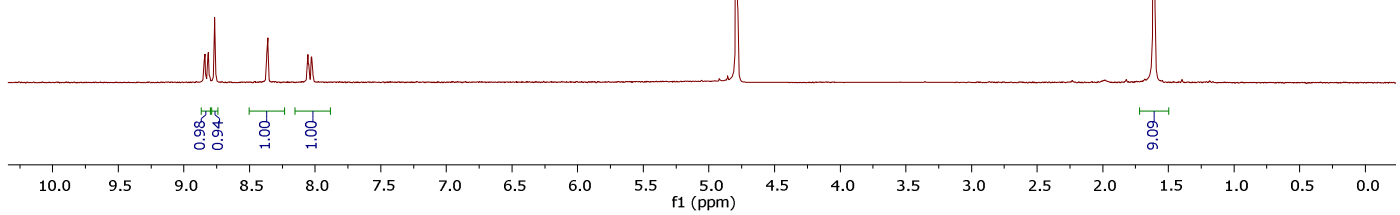

